TESIS DOCTORAL

Universidad Politécnica de Valencia

E.T.S.I.C.C.P. - Departamento de Hidráulica y Medio Ambiente Programa de doctorado: Ingeniería Hidráulica y Medio Ambiente

\title{
IMPLEMENTACIÓN DE UNA CLASIFICACIÓN ECO-HIDROLÓGICA DE LOS RÍOS DE CHILE Y SU APLICACIÓN A LA GESTIÓN AMBIENTAL
}

Tesis presentada por:

Matías Peredo Parada

Director:

Dr. Francisco Martínez Capel

Noviembre 2010 

A toda mí familia, por el apoyo que me han dado en estos largos años y en especial a mi gata loca.

Ñau. 

Si buscas resultados distintos

No hagas siempre lo mismo

(Albert Einstein) 



\section{Agradecimientos}

Me van a disculpar, pero esta vez no voy a agradecer, me da vergüenza hacerlo. Siento que mas bien debería pedir disculpas. Sí!, tal como lo leyó...disculpas!!!.

Por eso quiero disculparme ante toda la gente que me apoyó y me aguantó, y además sobrevivió. (A aquellos que no sobrevivieron, tarde o temprano nos veremos).

Quisiera empezar por pedir disculpas a mi tutor Francisco Martínez Capel, alias Paco Martínez Capel, o simplemente Paco. Disculpas por no poder desarrollar en mejor forma esta tesis (Uds. comprenderán que mi mente es limitada), por los malos ratos, por las decepciones que le he ocasionado y por todas las neuronas que le he matado o por lo menos atrofiado.

Aprovecho de disculparme ante mis evaluadores por haber tenido que soportar esta tesis tan humilde (pero empeñosa).

También quisiera pedir disculpas a los compañeros del equipo de investigación a Juan Diego, Aina, Virginia, Marta, Rui y Rafa sufridores de eternas y angustiosas salidas al campo (ojo, que en Chile, el campo quiere decir la huerta, entonces cuando me decían que íbamos a salir al campo a medir el caudal, mi limitada mente no podía comprender como en la huerta íbamos a medir este caudal). Disculparme amigos míos porque en ocasiones no les prestaba la atención, que personas como ustedes, se merecen; por 
privilegiar muchas veces lo científico a lo humano, por no haber compartido más tiempo con Uds., por no haberles podido entregado todo lo que, en circunstancias normales, me hubiese gustado.

Las disculpas alcanzan también para el resto de los amigos del doctorado a Ader, Ahmed, Diego Mauricio, Sandra, Chiara, Camilo, Catalina, Xitlali, Chucho, Miguel Ángel, Eduardo, Gabriel y Macarena.

A los otros amigos ajenos al programa de doctorado, pero de alguna forma vinculados. A Diegogo, Mipeten, Pedro Petrés, al Pastel, a las chicas del laboratorio, al glorioso equipo de Voleibol Nazaret, a mis queridas vecinas y su guardián Boludo.

Como no me voy a disculpar ante la persona que tuvo que soportarme en momentos difíciles, quien me apoyo en todo momento y es un soporte muy importante en mi vida. Gata loca de mi vida, discúlpame por no haberte correspondido de la manera como hubiese querido. Te quiero mucho, Amor mío

$\mathrm{Y}$ por último, quisiera disculparme ante mi familia, por los años que estuvimos separados, pero en mi corazón siento que en ningún momento estuvimos alejados.

Lo que sí quisiera agradecer es a la Agencia Española de Cooperación Internacional para el Desarrollo (AECID) quien me ha otorgado la beca de doctorado MAE-AECI necesaria para llevar a cabo esta tesis. 


\section{Tabla de Contenidos}

Agradecimientos 7

Tabla de Contenidos. 9

Resumen 13

Resum 17

Summary 21

Capitula1 Introducción. 23

Introducción 23

Bibliografía. 29

Capitula 2 Implementación REC-Chile. 31

Implementación de una clasificación Eco-Hidrológica en los ríos de Chile. 31

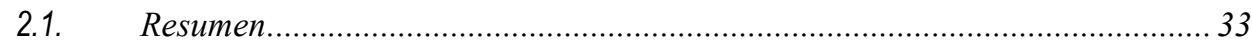

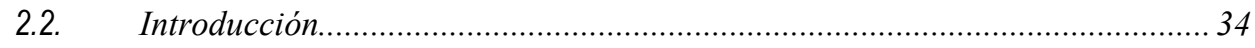

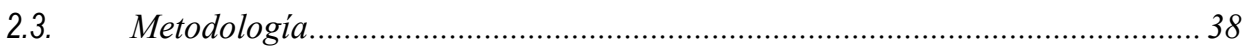

2.3.1. Factores controladores de la REC-Chile ................................................... 38

2.3.2. Implementación de la REC-Chile ............................................................ 40

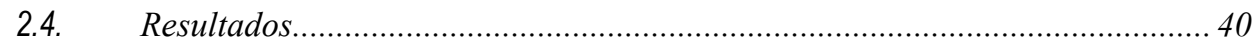

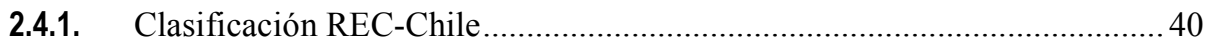

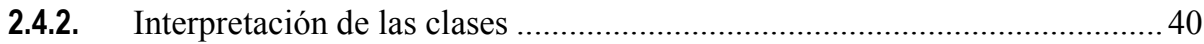

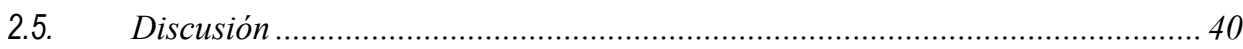

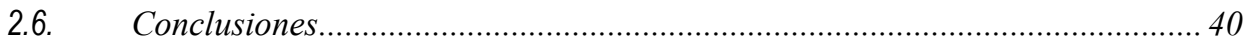




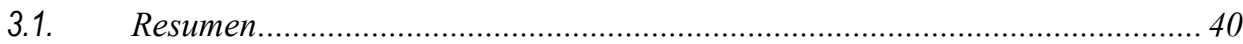

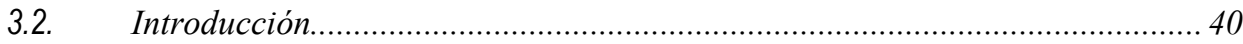

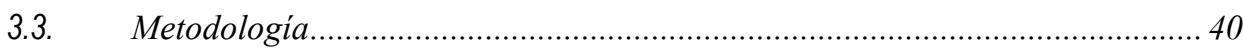

3.3.1. Evaluación hidrológica de la REC-Chile ................................................. 40

3.3.2. Fuentes de datos y variables del hidrograma ............................................ 40

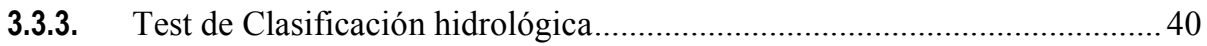

3.3.4. Interpretación hidrológica de las clases ..................................................... 40

3.3.5. Evaluación geoquímica de la REC-Chile ....................................................... 40

3.3.6. Datos y parámetros fisicoquímicos ................................................................... 40

3.3.7. Test de clasificación geoquímica ......................................................... 40

3.3.8. Interpretación geoquímica de las clases REC-Chile .................................... 40

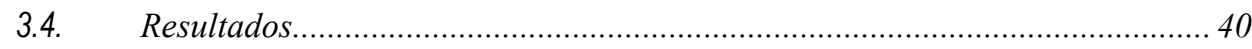

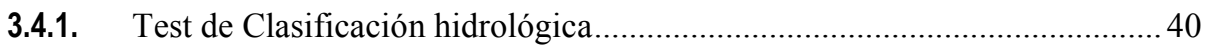

3.4.2. Interpretación hidrológica de las clases .................................................... 40

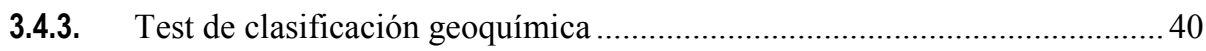

3.4.4. Interpretación geoquímica de las clases .................................................... 40

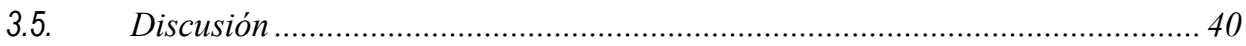

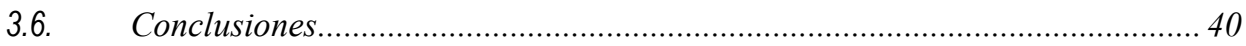

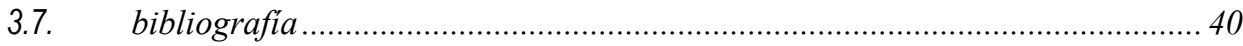

Capitula 4 Base de datos Eco-Hidrológica. .................................................................40

Base de datos Eco-Hidrológica de los ríos de Chile: una herramienta de gestión para

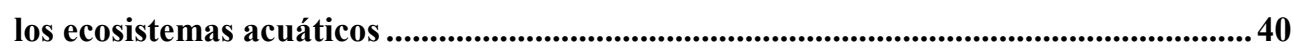

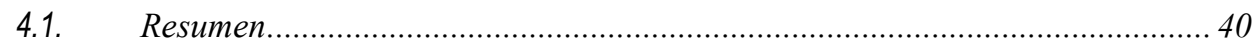

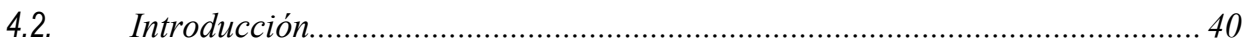




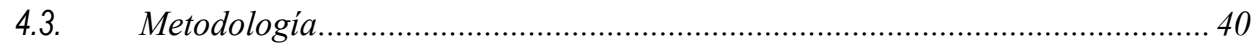

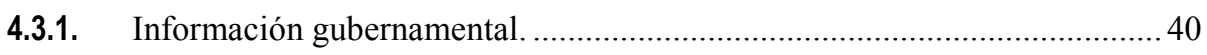

4.3.2. Información de fauna íctica.................................................................. 40

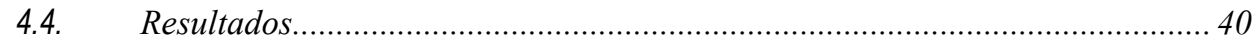

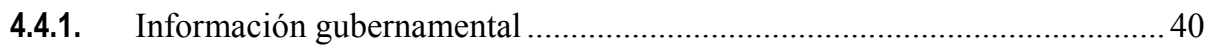

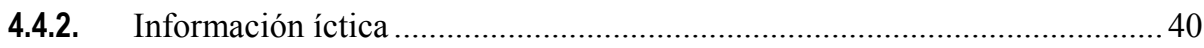

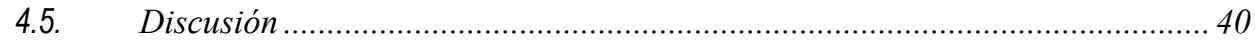

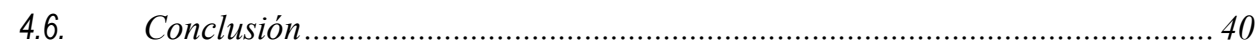

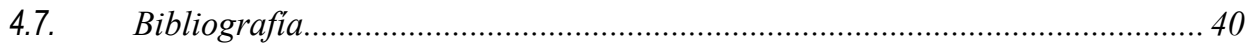

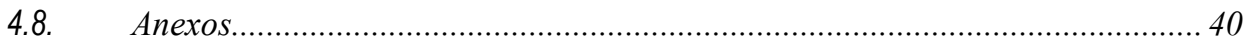

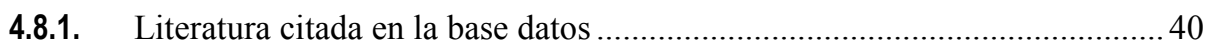

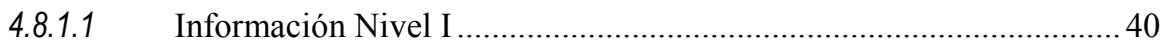

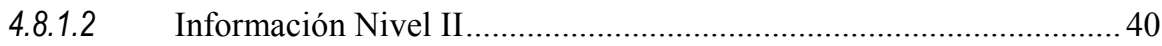

4.8.1.3 Información Nivel III ............................................................. 40

Capitulas Distribución geográfica potencial...........................................................40

Determinación de la distribución geográfica potencial de las especies dulceacuícolas 40

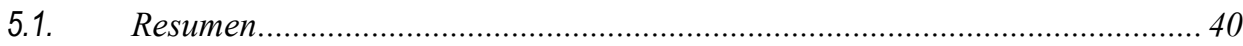

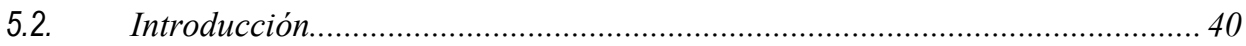

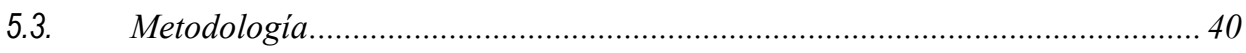

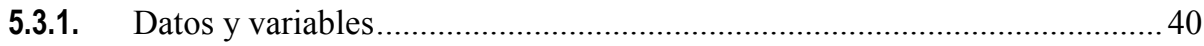

5.3.2. Evaluación de la clasificación REC-Chile ................................................ 40

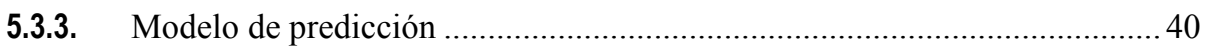

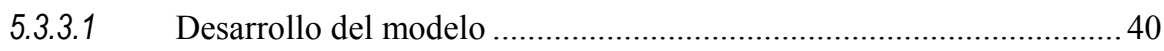

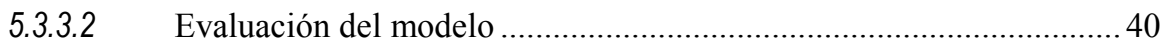

5.3.4. Rango de distribución geográfica ............................................................... 40

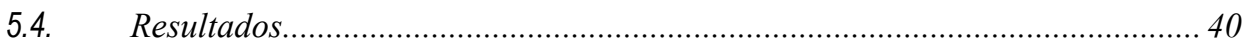

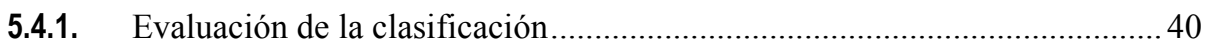

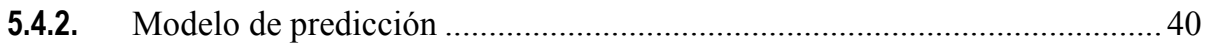




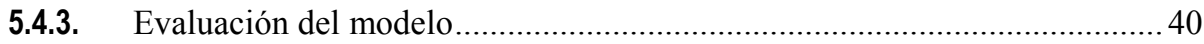

5.4.3.1 Importancia de las variables ......................................................... 40

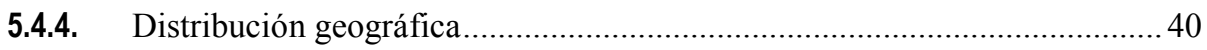

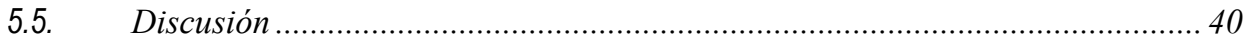

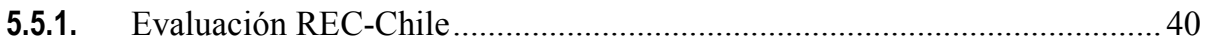

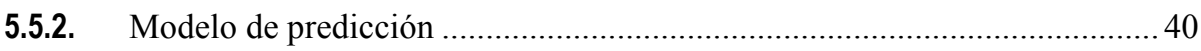

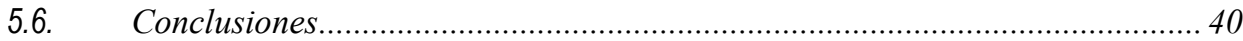

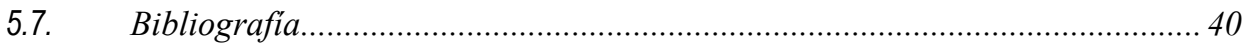

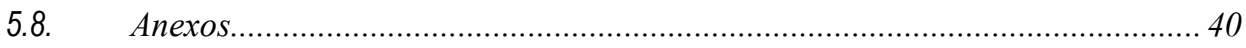

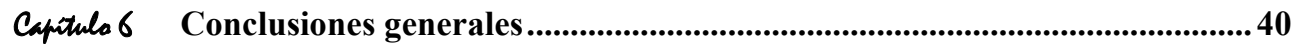

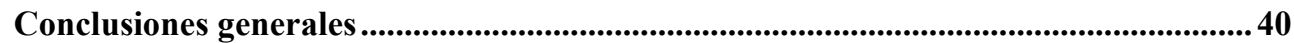




\section{Resumen}

En Chile existe un aumento en la preocupación por proteger y conservar los ecosistemas acuáticos por el gran valor ecológico que éstos presentan debido a su alto grado de endemismo de las especies, entre otros. Lamentablemente, las acciones antrópicas sobre estos ecosistemas producen presiones que han ido deteriorando sus hábitat. Sin embargo, no se ha desarrollado una herramienta que actúe como marco espacial para la planificación en la conservación y protección de estos ecosistemas. A ésto, se debe agregar que la información de las especies acuáticas es insuficiente, incompleta, fragmentada y poco actualizada.

Por ello se pensó que un sistema de clasificación, que incluyese aspectos climáticos, morfológicos, geológicos y que además permita trabajar bajo diversas escalas espaciales, podría ser una herramienta adecuada para suplir esta falta de marco espacial y escasez de información.

Existen varios tipos de clasificación, entre los cuales destacan las clasificaciones desarrolladas a partir de la deducción de factores controladores de los procesos fluviales, también denominadas como clasificaciones a priori, y aquellas clasificaciones desarrolladas a partir de datos, denominadas clasificaciones a posteriori. Entre las ventajas que presentan las clasificaciones a priori se encuentran la factibilidad de implementarlas en zonas con poca información, la interpretación de sus clases y la posibilidad de extrapolar su información a zonas desprovistas de ésta. 
Entendiendo que el caudal es la principal variable directora de la composición de ecosistemas fluviales, se ha desarrollado la clasificación Eco-Hidrológica de los ríos de Chile (REC-Chile) de tipo a priori, basada en la superposición jerárquica de los factores controladores: Clima, Origen de Flujo, Geología, Posición Relativa de Subsubcuenca, Uso de Suelo y Pendiente del tramo. Esta clasificación es multiescalar dotándola de una versatilidad que permite, según los factores controladores seleccionados, clasificar los tramos de río bajo distintos patrones fluviales y a diversas escalas espaciales. La evaluación de esta clasificación, así como la interpretación de sus clases, se realizó para objetivos hidrológicos, geoquímicos y biológicos

Para facilitar que la REC-Chile se convierta en una plataforma de gestión de los ecosistemas fluviales, se desarrollaron dos ejemplos de gestión y conservación de los ecosistemas fluviales. El primero, corresponde a la elaboración de una base de datos Eco-hidrológica, en donde se recopiló información fluviométrica, fisicoquímica y sedimentológica de los tramos de ríos, así como muestreos de especies ícticas nativas realizadas a distintas escalas espaciales. El segundo, corresponde a la determinación de la distribución geográfica potencial de las especies ícticas nativas mediante Modelos de Distribución Espacial, en los cuales se utilizaron las clases REC-Chile como variables de entrada a estos modelos.

Los resultados de la evaluación e interpretación de las clases, permiten concluir que la REC-Chile es una adecuada clasificación para los ríos de Chile y que además, ésta puede constituirse en un marco espacial para la 
RESUMEN

gestión y planificación de ecosistemas fluviales como lo muestran los resultados de los modelos de distribución espacial. 



\section{Resum}

A Xile, existeix un augment de la preocupació per protegir i conservar els ecosistemes aquàtics, ja que aquests presenten un elevat valor ecològic, a causa, principalment, de l'alt grau d'endemisme de les espècies que hi els habiten. Lamentablement, les accions antròpiques sobre aquests ecosistemes produeixen pressions que han anat deteriorant els seus hàbitats. No obstant això, no s'ha desenvolupat una eina que actue com a marc espacial per a la planificació en la conservació i protecció d'aquests ecosistemes. A tot això, cal afegir que la informació de les espècies aquàtiques és insuficient, incompleta, fragmentada i poc actualitzada.

Per la qual cosa, es va pensar que un sistema de classificació que inclogués aspectes climàtics, morfològics, geològics i que, a més, permetés treballar sota diverses escales espacials, podria ser una eina adequada per a suplir aquesta manca de marc espacial i d'informació.

Existeixen, doncs, diversos tipus de classificació, dintre de les quals destaquen, d'una banda, les classificacions desenvolupades a partir de la deducció de factors controladors dels processos fluvials, també denominades com classificacions a priori, i d'altra banda, aquelles classificacions desenvolupades a partir de dades, anomenades classificacions a posteriori. Entre els avantatges que presenten les classificacions a priori, existeix el fet de poder implementar, en zones amb poca informació, la interpretació de les seues classes i la possibilitat d'extrapolar la seua informació a zones desproveïdes d'aquesta. 
RESUM

Donat que el cabal és la principal variable directora de la composició d'ecosistemes fluvials, s'ha desenvolupat en aquesta tesi la classificació EcoHidrològica dels rius de Xile (REC-Chile) de tipus a priori basada en una superposició jeràrquica dels factors controladors del patró hidrològic. Aquesta classificació està realitzada a múltiple escala i està dotada d'una versatilitat que permet, segons els factors controladors seleccionats, classificar els trams de riu en funció de diferents patrons fluvials i diverses escales espacials. L'avaluació d'aquesta classificació, així com la interpretació de les seues classes, es va realitzar per a objectius hidrològics i geoquímics.

Així mateix, per facilitar que la REC-Chile es convertisca en una plataforma de gestió dels ecosistemes fluvials, es van desenvolupar dos exemples de gestió i conservació dels ecosistemes fluvials. El primer, correspon a l'elaboració d'una base de dades Eco-hidrològica, on es va recopilar informació fluviomètrica, fisicoquímica i sedimentològica dels trams dels rius estudiats, així com mostratges d'espècies íctiques natives realitzades segons diferents escales espacials. El segon exemple de gestió i conservació dels ecosistemes fluvials, correspon a la determinació de la distribució geogràfica potencial de les espècies íctiques natives mitjançant Models de Distribució Espacial, dins dels quals es van utilitzar les classes REC-Chile com a variables d'entrada a aquests models.

Els resultats de l'avaluació i interpretació de les classes, permeten concloure que la REC-Chile és una adequada classificació per als rius de Xile i que a més a més, aquesta pot constituir un marc espacial per a la planificació 
REsUm

d'ecosistemes fluvials, com ha quedat palès amb els resultats dels models de distribució espacial. 



\section{Summary}

In Chile there has been an increasing concern about the protection and management of aquatic ecosystems due to their high ecological value, mainly because of its high endemism degree of its species. Unfortunately, the anthropic actions exerted upon these ecosystems have brought forth pressures that have deteriorated their habitats. Despite this, there has not been developed a tool which acts as a spatial framework for conservation and management of aquatic ecosystems. In addition, information on aquatic species is insufficient, incomplete, scattered and not updated.

Due to the above stated, it was considered a classication system which included climatic, morphologic and geologic factors and that also allows to work under different spatial scales. Such a tool would be appropiated to provide the spatial framework and lack of information required for an adecuate management and protection of aquatic ecosystems.

Different types of classications exist of which stand out those developed from the deduction of controllling factors of the fluvial processes, also called a priori classifications, and classifications developed from data called a posteriori classifications. Among the advantages of the a priori classifications, there are the feasibility to set up in areas with scarce information, the interpretation of its classes and the possibility of extrapolate its information to other areas in which lack this information.

On the basis that the river flow is the main key driver of fluvial ecosystem, it has been developed the Eco-Hydrological Classification of Chilean rivers 


\section{SUMMARY}

(REC-Chile) an a priori type of classification based on a hierarchical superposition of the controlling factors of the hydraulic pattern. This classification is multiscalar provided with a versatility that allows, depending on the controlling factors selected, to classify a river reach according to different fluvial patterns and different spatial scales. The assessment of this classification, as well as the interpretation of their classes, was carried out for hydrological and geochemical purposes.

To facilitate that REC-Chile becomes in a management tool of fluvial ecosystems, it were developed two examples of management and conservation. The first one corresponds to the development of an ecohydrological database in which it were compiled fluvial, physicochemical, sedimentological data of the river reaches and fish sampling point. Information carried out at different spatial scales. The second example corresponds to the determination of the potential geographical distribution of native fish by means of Species Distribution Models in which were utilized the REC-Chile classes as input variables to these models.

Results of the evaluation and interpretation of the classes, allow to deduce that REC-Chile it seems to be an adequate classification system for chilean rivers and that in addition, it may constitute in a spatial framework for fluvial ecosystems as point out the results of spatial distribution patterns. 
Capitula 1

\section{Introducción}



CAPÍTULO 1 INTRODUCCIÓN Las presiones antrópicas hacia los ecosistemas acuáticos aumentan día a día en Chile. Como resultado de esto, las comunidades dulceacuícolas han visto deteriorado su hábitat (Orrego et al. 2009, Habit et al. 2006, Vila et al. 1999). La preocupación por parte del gobierno y entidades privadas para proteger estos ecosistemas ha ido en aumento debido al elevado valor ecológico del ecosistema, dado principalmente por el alto grado de endemismo de sus especies (Camousseight 2006, Habit et al. 2006, Jara et al. 2006, Ortíz \& Díaz-Páez 2006, Parada \& Peredo 2006, Valdovinos 2006, Vera \& Camousseight 2006). Sin embargo, no se ha desarrollado una herramienta que actúe como marco espacial para la planificación en la conservación y protección de estos ecosistemas.

Por ello se pensó que un sistema de clasificación que incluyese aspectos climáticos, morfológicos y geológicos y que además permita trabajar bajo diversas escalas espaciales, podría ser una herramienta adecuada para suplir esta falta de marco espacial para la gestión ambiental de los ríos. Para facilitar el uso de esta herramienta, se decidió implementarla en un Sistema de Información Geográfica.

Otros países han tenido y tienen en la actualidad la misma problemática. Entre ellos se destaca a Nueva Zelanda que presenta características geomorfológicas, orográficas y climáticas similares a las de Chile. En dicho país se han desarrollado clasificaciones medioambientales jerárquicas de ríos a priori (Snelder \& Biggs 2002). Estas clasificaciones aportan la ventaja de agrupar segmentos de río con su cuenca vertiente en unidades idóneas para la gestión ambiental, para el estudio geográfico de la distribución de la biota, y con una estructura jerárquica, que integra las 
CAPÍTULO 1INTRODUCCIÓN

cualidades hidrológicas, hidráulicas y ecológicas de una manera coherente con el principio del continuo fluvial. Esta clasificación no solo resulta idónea como base para el desarrollo y planificación de estudios ecológicos, sino también para el estudio del régimen ecológico de caudales y para la gestión de los ecosistemas fluviales.

Además del desarrollo de dicha clasificación, para facilitar que esta herramienta pueda convertirse en una plataforma de gestión y planificación ambiental de los ríos, y para no quedarnos solo en la teoría, se realizaron dos aplicaciones de conservación y distribución de especies nativas piscícolas, utilizando como figura principal la clasificación desarrollada.

Los ejemplos de aplicación tienen dos orientaciones; el primero plantea un primer paso en la gestión de los ríos, el cual es integrar toda la información disponible en un único marco de trabajo, como es la base de datos ecohidrológica de los ríos chilenos. El segundo ejemplo está más orientado a la gestión y conservación de las especies endémicas, al aplicar esta información en la determinación de modelos de distribución espacial de especies ícticas nativas.

La tesis que se presenta contiene 4 capítulos sobre el desarrollo y aplicación de la clasificación, y un sexto y último capítulo referente a las conclusiones generales de la tesis. Los capítulos tienen una estructura de artículos de difusión científica, es decir, cada uno de ellos presenta un(os) objetivo(s) e hipótesis de trabajo sobre los cuales se plantea la revisión de antecedentes, motivación del estudio, metodología utilizada, resultados, discusiones sobre estos resultados de acuerdo al objetivo planteado y finalmente una 

conclusión. En resumen, cada capítulo cuenta con su resumen, introducción, metodología, resultados, discusión, conclusión, bibliografía y anexos, en caso de tenerlos.

Cada uno de ellos corresponde a artículos de investigación realizados por el autor (uno ya publicado, otro aceptado para publicación, el resto en elaboración), que para dar una mayor coherencia, facilidad de lectura, y potencial difusión en países de habla hispana, han sido escritos todos en castellano, para la presentación de esta tesis. Debido a esta estructura de capítulos independientes, esta tesis cuenta con una introducción, pero no se ha considerado necesario extenderla con unos antecedentes, que producirían sin duda redundancias en el documento final. La línea argumental de estos trabajos, que forman esta tesis, se explica a continuación.

En el capítulo 2 se presenta la Clasificación Eco-Hidrológica para los ríos de Chile. En él, se detalla la elección del tipo de clasificación utilizada, sus ventajas e inconvenientes, las variables ambientales seleccionadas y su división en categorías.

En el capítulo 3 se realiza una evaluación de la clasificación, con el propósito de cuantificar si ésta resulta ser una correcta clasificación de los ríos. Además para facilitar las aplicaciones de esta clasificación se interpretaron sus clases. La evaluación e interpretación de las clases se realizó para objetivos hidrológicos y geoquímicos.

En el cuarto capítulo se muestra el primer ejemplo de aplicación. Éste corresponde a una base de datos eco-hidrológica, en donde se recopiló 
CAPÍTULO 1INTRODUCCIÓN

información hidrológica, fisicoquímica y sedimentológica de las estaciones de aforo administradas por el gobierno de Chile, así como información publicada sobre muestreos de especies ícticas nativas. La multiescalaridad de la clasificación desarrollada permite integrar toda esta información, que presenta diferentes escalas espaciales

El quinto capítulo es otro ejemplo de aplicación en la gestión ambiental de cuencas. Aquí se implementaron modelos de distribución espacial de las especies ícticas nativas usando como variables de entrada al modelo la información contenida en las clases de la clasificación. Los datos fueron obtenidos de la base de datos desarrollada en el capítulo anterior.

Finalmente, en el sexto y último capítulo se detallan las conclusiones generales de la clasificación, comentarios de su utilización, y su potencialidad como una plataforma a tener en cuenta para gestionar y planificar con criterios ecológicos en las cuencas hidrográficas en Chile. 


\section{Bibliografía}

CAMOUSSEIGHT A. 2006. Estado de conocimiento de los Ephemeroptera de Chile. Gayana 70(1): 50-56.

HABIT E, B DYER \& I VILA. 2006. Estado de conocimiento de los peces dulceacuícolas de Chile. Gayana 70 (11): 100-112.

JARA CG, EH RUDOLPH \& ER RIQUELME. 2006. Estado de conocimiento de los malacostracos dulceacuícolas de Chile. Gayana 70(1): 40-49.

ORREGO R, S MARSHALL, R BARRA, G CHIANG \& JF GAVILÁN. 2009. Patterns of fish community composition along a river affected by agricultural and urban disturbance in south-central Chile. Hidrobiología 620:35-46.

ORTÍZ JC \& H DÍAZ-PAEZ. 2006. Estado de conocimiento de los anfibios de Chile. Gayana 70(1): 114-121.

PARADA E \& S PEREDO. 2006. Estado de conocimiento de los bivalvos dulceacuícolas de Chile. Gayana 70(1): 82-87.

SNELDER T \& B BIGGS. 2002. Multiscale River Enviroment Classification for Water Resources Management. American Water Resources Association 38 (5): 1225-1239.

VALDOVINOS C. 2006. Estado de conocimiento de los gastrópodos dulceacuícolas de Chile. Gayana 70(1): 88-95.

VERA A \& A CAMOUSSEIGHT. 2006. Estado de conocimiento de los plecópteros de Chile. Gayana 70(1): 57-64.

VILA I, L FUENTES \& M CONTRERAS. 1999. Peces límnicos de Chile. Boletín Museo de Historia Natural, Chile 48: 61-75. 

Capítula 2 Implementación de una clasificación Eco-Hidrológica en los ríos de Chile. 



\subsection{RESUMEN}

En Chile existe un aumento en la preocupación por proteger y conservar los ecosistemas acuáticos debido al elevado estado de deterioro del hábitat. Como un primer paso para desarrollar planes de conservación se hace necesario realizar una clasificación de estos ecosistemas para conocer y entender los distintos tipos de ecosistemas y $\mathrm{su}$ funcionamiento. Entendiendo que el caudal es el principal factor en la composición de ecosistemas fluviales, se ha desarrollado una clasificación Eco-Hidrológica en los ríos de Chile (REC-Chile) basada en una superposición jerárquica de los factores controladores del patrón hidrológico en Chile.

La REC-Chile es multiescalar permitiendo, según los factores controladores seleccionados, representar distintos patrones fluviales a diversas escalas espaciales. La tipología del tramo de río se representa como un código de 6 dígitos, en donde la posición del dígito representa al factor controlador y el valor a la categoría de éste. Esta configuración jerárquica, así como la asignación en forma geográficamente independiente de sus clases, dota a la REC-Chile de una facilidad en la interpretación hidrológica de las clases.

Debido a la flexibilidad, entregada por la multiescalaridad, y por la sencilla interpretación de sus clases se espera que la REC-Chile se convierta en una herramienta en la gestión y desarrollo de planes de conservación de los ecosistemas acuáticos. 


\subsection{INTRODUCCIÓN}

La preocupación por el manejo sostenible de los cuerpos de aguas continentales es cada vez mayor a nivel mundial, tanto en el ámbito público como privado. Chile no está ajeno a ello, dado que sus ecosistemas fluviales distribuidos a lo largo de todo el país tienen un valor único, entre otros, por el alto grado de endemismo de sus especies. En vertebrados, el endemismo reportado a la fecha es de $81 \%$ en peces (Habit et al. 2006) y de 60,7\% en anfibios (Ortíz \& Díaz-Páez 2006). En invertebrados se ha reportado un endemismo de $91,7 \%$ en gastrópodos (Valdovinos 2006), 30,8\% en bivalvos (Parada \& Peredo 2006), 74,4\% en malacostracos (Jara et al. 2006), 56\% en efemerópteros (Camousseight 2006) y 57\% en plecópteros (Vera \& Camousseight 2006). Además, dado el creciente deterioro y/o fragmentación de los hábitat por causas antropogénicas, actualmente las especies dulceacuícolas presentan importantes problemas de conservación (Habit et al. 2006, Ortíz \& Díaz-Páez 2006, Parada \& Peredo 2006, Valdovinos 2006, Pérez-Losada et al. 2002, Bahamonde et al. 1998).

A la fecha, existe poca información respecto a medidas de conservación de las especies dulceacuícolas chilenas (Peredo et al. 2006, Peredo et al. 2005, Parada \& Peredo 2005, Habit et al. 2002) así como de conservación de sus hábitat (Habit et al. 2006). De igual modo, estos estudios carecen en su mayoría de los valores exactos de distribución local (Habit et al. 2006, Parada \& Peredo 2006, Valdovinos 2006), los cuales son antecedentes indispensables para llevar a cabo programas de gestión y conservación de las especies dulceacuícolas de Chile y del ecosistema fluvial en general. 
Como una medida de protección y conservación de los ecosistemas acuáticos el gobierno de Chile desarrolló la Estrategia Nacional de Cuencas Hidrográficas (ENGICH) que busca contribuir al uso sustentable de los recursos hídricos, armonizando la protección de los ecosistemas con la disponibilidad del recurso (CONAMA 2007). Para lograr una adecuada implementación de la ENGICH, se hace necesario conocer y clasificar los cuerpos de aguas existentes en el país.

Las clasificaciones del territorio han sido desarrolladas para propósitos de gestión como una herramienta para el entendimiento de los ecosistemas. Permiten la interpretación de datos, el desarrollo de inventarios, el avance en el conocimiento de cuencas no monitoreadas mediante la extrapolación desde cuencas monitoreadas, determinación de estados de referencias, etc. (Omernik \& Bailey 1997, Bailey 1995).

Existen diversos métodos para realizar una clasificación de los sistemas naturales acuáticos, entre ellos destacan las clasificaciones taxonómicas y aquellas basadas en la regionalización del territorio. Las taxonómicas no necesariamente se basan en procesos físicos o ecológicos que rigen los ecosistemas acuáticos, mientras que aquellas basadas en la regionalización, también conocidos como ecoregiones, responden a un enfoque físico que considera las variables que determinan los patrones que condicionan los procesos fluviales (González del Tánago \& García de Jalón 2006, Bailey et al. 1978), resultando zonas homogéneas respecto a ciertas características o parámetros a una determinada escala espacial (Snelder et al. 2005, Omernik \& Bailey 1997, Bailey et al. 1978). Snelder \& Biggs (2002) señalan que 
CAPÍTULO 2 IMPLEMENTACIÓN REC-CHILE

estas ecoregiones son incapaces de representar gradientes longitudinales a lo largo del río. Estos gradientes son reconocidos que ocurren en un ecosistema fluvial como el concepto de river continuum (Vannote et al. 1980), por lo que se verían limitadas para realizar una clasificación de ríos.

A partir de los años 80, diversos autores han abordado las clasificaciones del territorio considerando los sistemas de organización jerárquica de los ríos. Reconocen que los ríos pertenecen a una cuenca vertiente y por lo tanto, éstos se ven influenciados por las características que lo rodean, asumiendo que los procesos ecológicos en un río dependen de factores físicos que ocurren a varias escalas. (González del Tánago \& García de Jalón 2006, Snelder \& Biggs 2002, Montgomery \& Buffington 1997, Omernik \& Bailey 1997, Frissell et al. 1986,).

Snelder \& Biggs (2002) desarrollaron la River Environment Classification (REC) para clasificar los ríos de Nueva Zelanda basada en las siguiente premisas a) Los patrones ecológicos son dependiente de un conjunto de factores asociados a la escala regional de diversos procesos físicos. b) Las características ecológicas de los ríos responden a los procesos fluviales y c) Asigna en forma geográficamente independiente las clases a cada tramo de río. En la REC, la tipología de cada tramo de río se determina mediante una superposición jerárquica de factores controladores, causantes principales de la variación espacial del patrón hidrológico de un río a una escala determinada (Snelder et al. 2005, Snelder \& Biggs 2002).

Dado que existen algunas similitudes eco-hidrológicas entre los ríos de Chile y Nueva Zelanda (régimen hidrológico y la geomorfología de los ríos) 
CAPÍTULO 2 IMPLEMENTACIÓN REC-CHILE y también taxocenóticas por la presencia de Galaxias maculatus y Geotria australis (Vila et al. 2006, Dyer 2000, Mc Dowall 2000) y de numerosas especies de macroinvertebrados (Winterbourn 1981), hipotetizamos que dichas similitudes permitirían adaptar una REC para Chile, teniendo en consideración las diferencias geomorfológicas y climáticas existentes, esperando que se constituya en una herramienta de gestión de los ecosistemas fluviales chilenos.

Los objetivos de presente capítulo son 2, implementar una REC en Chile (REC-Chile) e interpretar algunas de sus clases para su aplicación a la gestión y planificación de los ecosistemas fluviales. 


\subsection{METODOLOGÍA}

\subsubsection{Factores controladores de la REC-Chile}

Los factores controladores seleccionados para implementar la REC-Chile están basados en aquellos utilizados en la REC desarrollada por Snelder y Biggs (2002) para Nueva Zelanda, adaptados a las condiciones climáticas y ambientales de Chile. Los factores seleccionados fueron: Clima, Origen de Flujo, Geología, Posición Relativa de Subsubcuenca, Uso del suelo y Pendiente del tramo.

En la jerarquía de la REC-Chile, el Clima es el único factor controlador a macroescala. Determina principalmente las características hidrológicas, como la magnitud del flujo así como la frecuencia de avenidas y de caudales bajos (Snelder et al. 2005, Snelder \& Biggs 2002). Sus categorías fueron Árido, Semiárido, Subhúmedo, Húmedo y Muy húmedo, y se basan en la clasificación climática de Blair (Heras 1973).

El factor Origen de Flujo actúa a un nivel de mesoescala, e indica la fuente o procedencia del flujo que puede ser pluvial, nival, glaciar o una combinación de ellas (Fleming 2005, Snelder et al. 2004a). Está relacionado con la estacionalidad del flujo y el transporte de sedimentos a escala de subcuenca (Snelder \& Biggs 2002). La precipitación en Chile procede principalmente de efectos orográficos, pues la cordillera de los Andes actúa como barrera para los vientos húmedos del Pacífico (Villagrán \& Hinojosa 
CAPÍTULO 2 IMPLEMENTACIÓN REC-CHILE 2005, Rutllant 2004). Este hecho permite asociar a cada subcuenca, según su altitud, la proporción de precipitación que aporta respecto a la cuenca total.

Este factor comprende cinco categorías de origen topográfico (Llano, Valle, Precordillera, Cordillera y Nieves eternas) y tres categorías asignadas manualmente (Lagos, Glaciares y Regulaciones). La categoría Llano se refiere a subcuencas que se encuentran bajo los $300 \mathrm{msnm}$. Estas categorías presentan una precipitación acumulada baja, menores al 25\% del total de la cuenca, y variaciones estacionales extremas entre verano e invierno. Valle se corresponde con subcuencas con una altitud comprendida entre 300 y $1000 \mathrm{msnm}$ y con porcentajes medios de precipitación acumulada entre un $25 \%$ y un $40 \%$ del total de la cuenca, resultando un caudal anual medio mayor y variaciones estacionales más moderadas. La categoría Precordillera representa a cuencas entre los 1000 y $2400 \mathrm{msnm}$, presentando altos porcentajes de precipitación acumulada (entre un $40 \%$ y un $70 \%$ ) e incluye la contribución por derretimiento de nieve durante la primavera-verano; esto provoca la formación de un caudal pico mayor en invierno (periodo de lluvias) y otro menor en primavera-verano (periodo de sequía). Cordillera corresponde a subcuencas situadas entre 2400 y 4200 msnm, caracterizándose por una gran contribución del derretimiento de nieve y pequeños aportes de precipitación, por lo que el régimen del flujo presenta un pico menor en invierno y otro mayor a comienzos del verano. Nieves eternas son subcuencas por encima de $4200 \mathrm{msnm}$. Solo recibe derretimiento de nieve, presentando grandes caudales en verano y bajos en invierno. 
CAPÍtUlo 2 IMPLEMENTACIÓN REC-CHILE

Dentro de las categorías asignadas manualmente, la categoría Glaciar se asigna a cualquier tramo de río cuya subcuenca contenga un glaciar con un área superior al 20\%. Lagos agrupa masas de aguas que modifican el régimen de caudales reduciendo o desfasando los caudales extremos. Para evaluar si se produce dicha modificación, se calculó el Lake Index (LI) (ecuación 2.1) que estima la precipitación sobre el lago respecto a la cuenca drenante del tramo de río.

$$
\boldsymbol{L} \boldsymbol{I}=\frac{\sum \boldsymbol{V}_{L W} \cdot \frac{\sum \boldsymbol{A}_{L}}{\sum \boldsymbol{A}_{L W}}}{\boldsymbol{V}_{W}}
$$

Donde $A_{L}$ es el área del lago, $A_{L W}$ es el área de captación del lago, $V_{\mathrm{LW}}$ es la precipitación media anual en el área de captación de lago, y $\mathrm{V}_{\mathrm{W}}$ es la precipitación anual en la cuenca.

El LI evalúa si la influencia de las masas de agua representa una modificación significativa o no en el hidrograma de una subcuenca; fue propuesto por Snelder \& Biggs (2002) y modificado por Snelder (comunicación personal). Para determinar el valor umbral de LI en ríos chilenos, se compararon los hidrogramas anuales entre estaciones de aforo con el mismo código REC-Chile, con y sin presencia de un lago aguas arriba. Entre ellas se estimó un valor crítico de 0.020 , por tanto la regulación del lago es significativa con valores mayores a dicho umbral.

Dado que las categorías del factor Origen de Flujo se relacionan con patrones hidrológicos en régimen natural, fue necesario incorporar otra 
CAPITULO 2 IMPLEMENTACIÓN REC-CHILE categoría, para los tramos donde existen regulaciones antrópicas, por ejemplo, embalses; la categoría incorporada es Regulaciones.

El factor Geología se relaciona, a un nivel de mesoescala, con las características geoquímicas del agua dominadas por las propiedades geológicas del acuífero (Herrera et al. 2006, González et al. 1999). Un parámetro importante en las calidad de las aguas de los ríos de Chile son las sales disueltas debido al origen marino y/o volcánico de las cuencas (Vila \& Molina 2006), mostrando un pronunciado gradiente norte-sur. Las ocho categorías definidas para este factor fueron: Depósitos no consolidados, Rocas Plutónicas, Rocas Volcánicas, Rocas Sedimentarias con mezcla de rocas volcánicas, Rocas Sedimentarias, Rocas Volcánicas fracturadas y Rocas Carbonatadas. Se incorporó la categoría No reconocidas, cuando no fue posible establecer el origen geológico.

Posición Relativa de la Subsubcuenca es el cuarto factor controlador. Éste relaciona el área drenante en un tramo de río con respecto al total de su cuenca. Describe aspectos del patrón hidrológico como la magnitud del caudal medio, intensidad y atenuación de los caudales de avenidas, y el flujo de sedimentos. La regla de asignación se determinó según el porcentaje del área drenante con respecto a la cuenca, definiendo las siguientes categorías: Cabecera, Tramo Alto, Tramo Medio y Tramo Bajo. Las categorías Endorreica e Internacionales fueron asignadas manualmente.

El factor Uso del Suelo se relaciona con procesos a nivel de mesoescala como la intercepción inicial y los procesos de evapotranspiración, además controla aspectos fisicoquímicos de la calidad del agua producto de la 
CAPÍTULO 2 IMPLEMENTACIÓN REC-CHILE

lixiviación del suelo y arrastre del río (Sliva \& Dudley 2001, Johnson et al. 1997). Se han definido nueve categorías: Áreas urbanas, Agricultura, Praderas y matorrales, Bosque, Áreas sin vegetación, Cuerpos de agua, Nieves y glaciares y Áreas sin información.

El último factor controlador es la Pendiente del tramo de río. Describe patrones morfohidráulicos a un nivel de microescala tales como transporte local de sedimento, erosión del cauce, velocidad media del flujo e influencia de las condiciones de la ribera. La regla de asignación está determinada según la pendiente del tramo de río en tres categorías: Baja, Media y Alta.

\subsubsection{Implementación de la REC-Chile}

REC-Chile fue implementada mediante un Sistema de Información Geográfico (SIG) en ríos representados como una red hidrográfica digital a una escala 1:250.000. La información utilizada para desarrollar los factores controladores así como la red hidrográfica fue proporcionada por la Dirección General de Aguas (DGA), Ministerio de Obras Públicas, Chile. Esta información incluye líneas isoyetas, líneas de evapotranspiración potencial y real (1:250.000), curvas de nivel (1:250.000), áreas de subcuencas (1:50.000), áreas de cuencas (1:50.000), geología (1:50.000) y uso del suelo (1:50.000).

El mapeo de la REC-Chile se hizo de acuerdo a la escala espacial de cada factor controlador (escalamiento top-down) siguiendo las reglas de asignación definidas para cada categoría. En las categorías de los factores Clima, Origen de Flujo y Geología se consideró el concepto de river 
CAPÍTULO 2 IMPLEMENTACIÓN REC-CHILE continuum (Vannote 1980) propagando hacia aguas abajo las características de las categorías más influyentes. En el factor Clima, se propagaron 4 categorías que dominan la magnitud del caudal. El orden de propagación de las categorías fue: Semiárido, Subhúmedo, Húmedo y Muy Húmedo. En el factor Origen de Flujo, se propagaron las características estacionales del hidrograma. Primero se propagaron las categorías topográficas (Llano, Valle, Precordillera, Cordillera y Nieves eternas) y posteriormente se propagaron las categorías asignadas manualmente (Lagos, Glaciares y Regulaciones). En el factor Geología el criterio de propagación se realizó en base al aporte de sales disueltas de las distintas litologías del suelo. El orden de propagación de las categorías fue: rocas Plutónicas, rocas Volcánicas, rocas Sedimentarias con mezcla de volcánicas, rocas Sedimentarias, rocas Volcánicas fracturadas y por último rocas Carbonatadas

REC-Chile fue desarrollada en las cuencas comprendidas entre los $18^{\circ} \mathrm{S}$ y los $43^{\circ} \mathrm{S}$. En las cuencas más australes $\left(43^{\circ} \mathrm{S}\right.$ a $\left.55^{\circ} \mathrm{S}\right)$ la clasificación no fue implementada debido a la falta de información para definir los factores controladores.

Una vez diseñada e implementada la REC-Chile se realizaron dos aplicaciones simples para mostrar como interpretar el patrón hidrológico y la calidad natural geoquímica de las aguas. En el primer caso se compararon aspectos básicos del hidrograma anual en los tramos de río de 4 estaciones de aforo en régimen natural, o levemente modificados, mediante la codificación REC-Chile asociada a los tramos de río en donde se ubican estas estaciones de aforo. En el segundo caso se compararon los valores medios de conductividad eléctrica del agua (CE) en 67 tramos de río 
CAPÍTULO 2 IMPLEMENTACIÓN REC-CHILE

distribuidos en todo Chile con sus respectivas clases de la REC-Chile. Los

datos del hidrograma anual de las estaciones de aforo así como de CE fueron entregados por la DGA. 


\subsection{RESULTADOS}

\subsubsection{Clasificación REC-Chile}

En la Tabla 2.1 se presenta la REC-Chile con cada uno de los factores controladores y sus respectivas categorías, reglas de asignación y el código asociado a cada categoría.

La clasificación resultante presenta estructura de árbol en donde las categorías del i-ésimo factor se dividen en las categorías del factor i+1 y así sucesivamente. Los niveles de clasificación se definen de acuerdo a los factores controladores superpuestos jerárquicamente (Figura 2.1). El primer nivel de clasificación corresponde al primer factor controlador (Clima), el segundo nivel de clasificación es la superposición jerárquica de los dos primeros factores (Clima y Origen de Flujo), el tercer nivel se define al agregar al factor Geología al segundo nivel. Así sucesivamente hasta el sexto y último nivel de clasificación definido por la superposición jerárquica de todos los factores controladores de la REC-Chile. 


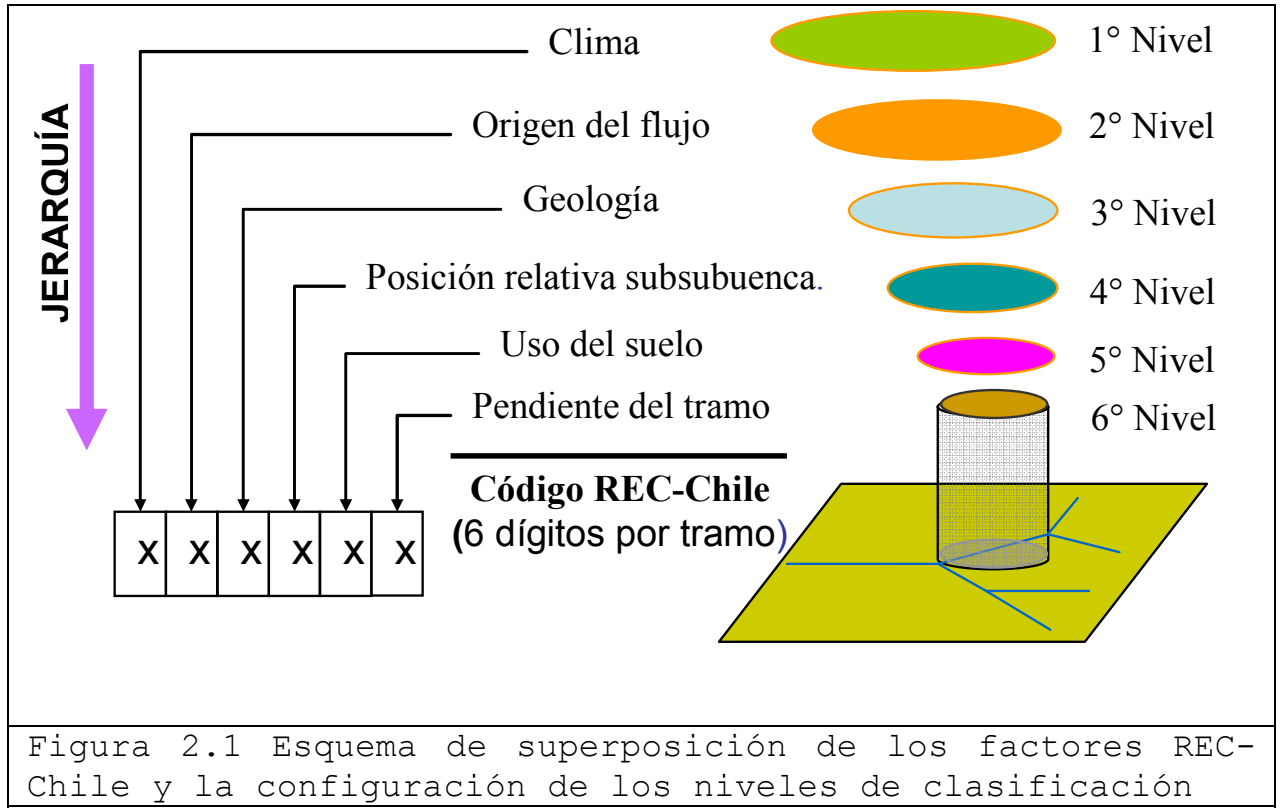

El sexto nivel de clasificación se refleja en un código de seis dígitos. La posición del dígito representa el nivel de clasificación, y el valor del dígito representa la categoría del factor controlador a ese nivel. 
Tabla 2.1.Niveles de clasificación de la REC-Chile, sus factores, categorías, códigos y reglas de asignación geográfica

\begin{tabular}{|c|c|c|c|c|}
\hline Factor & Categorías & & Código & Reglas de asignación geográfica \\
\hline \multirow[t]{5}{*}{ 1.-Clima } & Árido & (Ar) & 1000000 & Precipitación efectiva media anual $\leq 250 \mathrm{~mm}$. \\
\hline & Semiárido & $(\mathrm{Sa})$ & 2000000 & $250 \leq$ Precipitación efectiva media anual $<500 \mathrm{~mm}$. \\
\hline & Subhúmedo & $(\mathrm{Mw})$ & 3000000 & $500 \leq$ Precipitación efectiva media anual $<1000 \mathrm{~mm}$ \\
\hline & Húmedo & $(\mathrm{W})$ & 4000000 & $1000 \leq$ Precipitación efectiva media anual $<2000 \mathrm{~mm}$. \\
\hline & Muy húmedo & $(\mathrm{Vw})$ & 5000000 & $\geq 2000$ mm. Precipitación efectiva media anual. \\
\hline \multirow{8}{*}{$\begin{array}{l}\text { 2.-Origen de } \\
\text { Flujo }\end{array}$} & Llano & (L) & 10000 & Porcentaje de precip acumulada $\leq 25 \%$; elevación $\leq 300 \mathrm{msnm}$. \\
\hline & Valle & $(\mathrm{V})$ & 20000 & $25 \%<$ Precip. acumulada $\leq 40 \% ; 300<$ elevación $\leq 1000 \mathrm{msnm}$. \\
\hline & Precordillera & $(\mathrm{Mm})$ & 30000 & $40 \%<$ Precip. acumulada $\leq 75 \% ; 1000<$ elevación $\leq 2400 \mathrm{msnm}$. \\
\hline & Cordillera & $(\mathrm{M})$ & 40000 & $75 \%$ > Precip. acumulada.; $2400<$ elevación $\leq 4200$ msnm. \\
\hline & Nieves eternas & (Es) & 50000 & Sin precipitación líquida, elevación $\geq 4200$ msnm. \\
\hline & Lagos & La) & 60000 & Índice de influencia lacustre $(\mathrm{LI}) \geq 0.020$ \\
\hline & Regulaciones & $(\mathrm{Rg})$ & 70000 & Manualmente asignada. \\
\hline & Glaciar & $(\mathrm{G})$ & 80000 & Manualmente asignada. (Área glaciar sup.a 20\% área subcuenca) \\
\hline \multirow[t]{8}{*}{ 3.-Geología } & \multicolumn{2}{|c|}{ Depósitos no consolidados (Al) } & 1000 & \\
\hline & Plutónicas and hipabisal & $(\mathrm{Pl})$ & 2000 & \\
\hline & Volcánicas & $(\mathrm{Vc})$ & 3000 & \\
\hline & Sed. and mix sed-volc & $(\mathrm{Mx})$ & 4000 & \\
\hline & Metam. and sedimentaria & s $(\mathrm{Ms})$ & 5000 & \\
\hline & Volcánicas fracturadas & $(\mathrm{Vf})$ & 6000 & \\
\hline & Carbonatadas & $(\mathrm{Ca})$ & 7000 & \\
\hline & Área sin información & (Ni) & 8000 & \\
\hline
\end{tabular}


Tabla 2.1.Niveles de clasificación de la REC-Chile, sus factores, categorías, códigos y reglas de asignación geográfica

\begin{tabular}{|c|c|c|c|c|}
\hline Factor & Categorías & & Código & Reglas de asignación geográfica \\
\hline \multirow{6}{*}{$\begin{array}{l}\text { 4.-Posición } \\
\text { Relativa de } \\
\text { subsubcuenca }\end{array}$} & Cabecera & $(\mathrm{Hw})$ & 100 & Area acumulada $\leq 7.5 \%$ área de la cuenca. \\
\hline & Tramo alto & (Ur) & 200 & $7.5 \%<$ área acumulado $\leq 25 \%$ área de la cuenca. \\
\hline & Tramo medio & $(\mathrm{Mr})$ & 300 & $25 \%<$ área acumulada $\leq 70 \%$ área de la cuenca. \\
\hline & Tramo bajo & $(\mathrm{Lr})$ & 400 & Área acumulada $\geq 70 \%$ área de la cuenca. \\
\hline & Endorreicas & (E) & 500 & Cuencas sin drenaje al mar. \\
\hline & Internacionales & (I) & 600 & Cuencas internacionales. \\
\hline \multirow{9}{*}{$\begin{array}{l}\text { 5.-Uso } \\
\text { suelo }\end{array}$} & Áreas urbanas & $(\mathrm{Ci})$ & 10 & \\
\hline & Agricultura & $(\mathrm{Ag})$ & 20 & \\
\hline & Praderas y matorrales & (Ps) & 30 & \\
\hline & Bosques & (Wo) & 40 & \\
\hline & Área sin vegetación & $(\mathrm{Nv})$ & 50 & \\
\hline & Cuerpos de agua & $(\mathrm{R})$ & 60 & \\
\hline & Humedales & $(\mathrm{Wt})$ & 70 & \\
\hline & Nieves y glaciares & $(\mathrm{Sg})$ & 80 & \\
\hline & Área sin información & $(\mathrm{Ni})$ & 90 & \\
\hline \multirow{3}{*}{$\begin{array}{l}\text { 6.-Pendiente } \\
\text { del tramo }\end{array}$} & Alta & (Hi) & 1 & Pendiente $>0.04 \%$ \\
\hline & Media & $(\mathrm{Md})$ & 2 & $0.04 \% \leq$ pendiente $<0.02 \%$ \\
\hline & Baja & $(\mathrm{Lw})$ & 3 & Pendiente $\leq 0.02 \%$ \\
\hline
\end{tabular}


CAPÍTULO 2 IMPLEMENTACIÓN REC-CHILE Las clases se definen de acuerdo a la superposición jerárquica de las categorías de los factores del nivel seleccionado. Así, para el primer nivel las clases son las categorías del factor Clima, para el segundo nivel las clases resultantes son las categorías del factor Clima combinadas con las categorías del Origen de Flujo, es decir, Árido es combinada con las categorías Llano, Valle, Precordillera, Cordillera, Nieves eternas, Lagos, Regulaciones y Glaciar. Este procedimiento se repite para cada una de las categorías del factor Clima. Posteriormente cada una de estas clases se combina con las categorías del factor Geología conformando el tercer nivel de clasificación. Así sucesivamente hasta el factor Pendiente del Tramo, conformando el sexto y último nivel de clasificación.

El número potencial de clases en cualquier nivel de clasificación depende del número de categorías a este nivel y del número de clases de los niveles previos, por tanto, el número potencial de clases está dado por la combinación matemática del número de categorías de cada factor controlador. Por ejemplo, el segundo nivel tiene potencialmente 40 clases ( 5 x 8), el tercer nivel tiene potencialmente 320 clases $(5 \times 8 \times 8)$. En total, REC-Chile tiene 46080 potenciales clases, sin embargo, no todas las clases potenciales ocurren físicamente en una región; por ejemplo, en la región de la Araucanía (37³0' a 39³0’S), con un área aproximada a 22.000 km², sólo existen 515 clases de las 27.000 posibles para esta región. El número total de clases de la REC-Chile para el territorio en la cual fue implementada es de 3049 .

Debido a la escala espacial de cada factor controlador, las clases del nivel Clima definen áreas homogéneas más grandes que las clases del nivel 
CAPÍTULO 2 IMPLEMENTACIÓN REC-CHILE

Origen de Flujo, lo cual genera diferencias en la escala espacial de los patrones generados. La Figura 2.2 muestra la clasificación en los dos primeros niveles para dos cuencas ubicadas al norte de la Patagonia (Imperial y Toltén). En ella, se aprecia la propagación hacia aguas abajo de las categorías más influyentes sobre el cauce principal de ambos ríos. En el primer nivel (Figura 2.2b), se observa la propagación de la categoría Muy húmedo $(\mathrm{Vw})$ proveniente de las lluvias que ocurren en la Cordillera de los Andes. De la misma forma, en el segundo nivel (Figura 2.2c) se observa la propagación de la clase Muy húmedo/Precordillera $(\mathrm{Vw} / \mathrm{Mm})$ en el río Imperial y de la categoría Muy húmedo/Lagos $(\mathrm{Vw} / \mathrm{La})$ en el río Toltén. Esto significaría que el hidrograma del río Imperial debería tener aproximadamente una estacionalidad similar al régimen de precipitación que ocurre en las cercanías de la Cordillera de los Andes; en cambio, el hidrograma del río Toltén se vería influenciado en casi todo el recorrido por el efecto amortiguador del Lago Villarrica. 
CAPÍTULO 2 IMPLEMENTACIÓN REC-CHILE
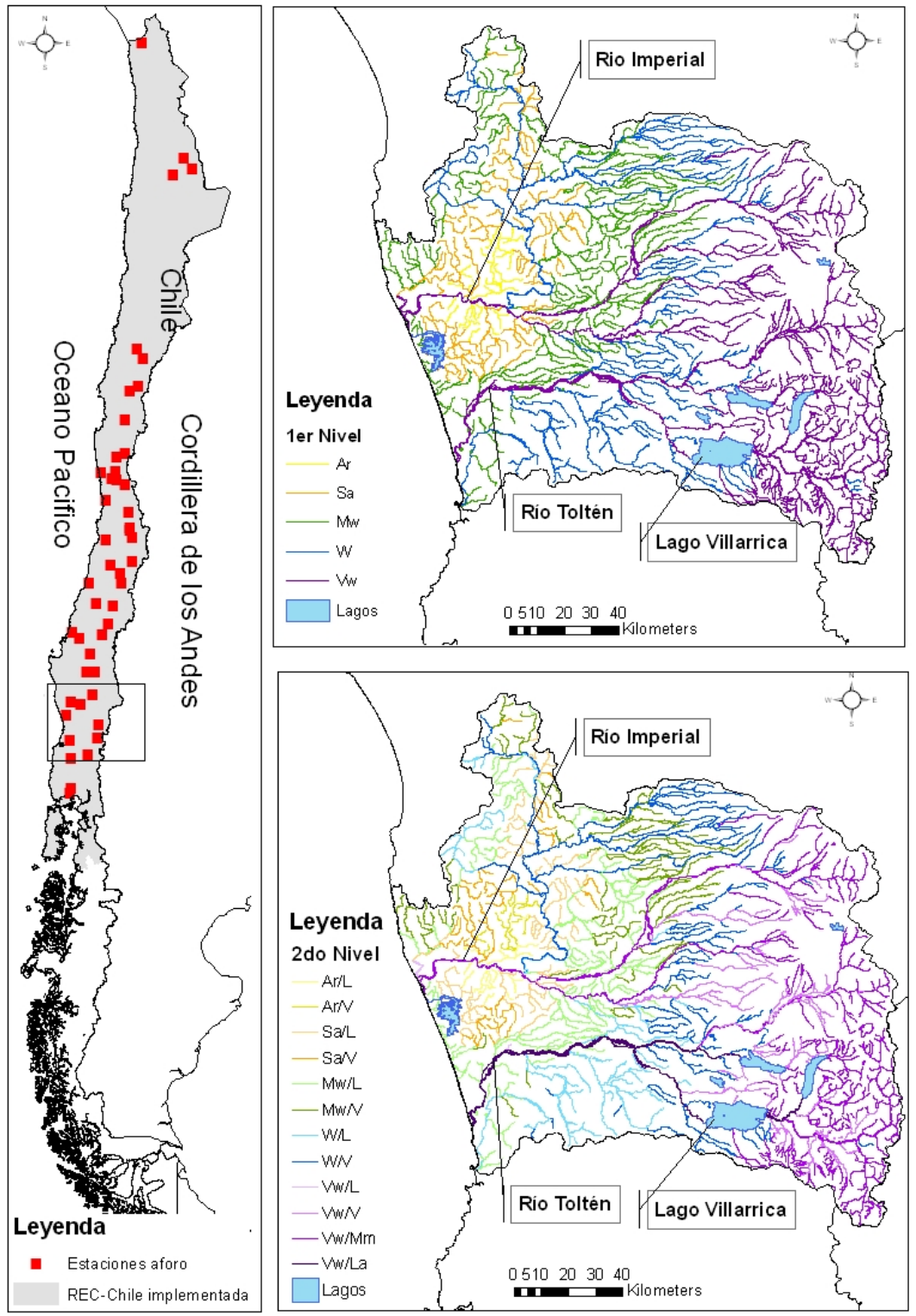

Figura 2.2. Rango geográfico en el cual la REC-Chile fue implementada (a). La figura superior (b) muestra el primer nivel de clasificación de la REC-Chile (factor Clima) en las cuencas del río Imperial y Toltén. La Figura inferior (c) muestra el segundo nivel de clasificación (factores Clima y Origen de Flujo) en las mismas cuencas. Mayor detalles en interpretación de las clases y su regla de asignación ver Tabla 2.1 
CAPITULO 2 IMPLEMENTACIÓN REC-CHILE

2.4.2. Interpretación de las clases

Combinando ciertos factores controladores es posible interpretar algunos patrones eco-hidrológicos del sistema fluvial. El segundo nivel de clasificación, es decir, superponiendo los factores controladores Clima y Origen de Flujo, permite determinar el orden de magnitud del caudal medio anual de un tramo de río en régimen natural, así como su variación estacional. Las clases de este nivel logran interpretar descriptivamente un hidrograma medio anual de tramos de ríos en condiciones de régimen natural. Se espera que las clases con categorías Árido y Semiárido presenten un bajo caudal medio anual. Dentro de estas categorías, las que contengan las aquellas de origen pluvial (Llano y Valle) se espera que presenten un flujo intermitente con variaciones estacionales extremas con respecto al caudal medio anual y que éstas ocurran en verano (caudales mínimos) y en invierno (caudales altos). Las que contengan la categoría Precordillera se espera que presenten un flujo intermitente con un hidrograma bimodal producto del aporte mixto de las precipitaciones y los derretimientos de nieve. Las categorías Cordillera y Nieves eternas tendrán variaciones estacionales moderadas con respecto al caudal medio anual, ocurriendo los caudales máximos en época de derretimiento de nieves y el resto del año un caudal base mínimo, de la misma forma se espera que la categoría Glaciar presente flujo por el porte del derretimiento de glaciares. La categoría Lagos no se encuentran en combinación con las categorías Árido y Semiárido.

La categoría Subhúmedo se espera que presente caudales medios anuales más altos que las dos categorías anteriores. La presencia de categorías pluviales presenta caudales continuos con variaciones estacionales que 
CAPÍTULO 2 IMPLEMENTACIÓN REC-CHILE responden al régimen pluviométrico. La categoría Precordillera, sigue presentando un hidrograma medio anual bimodal, pero con un caudal punta mayor en invierno con respecto a las categorías Árido y Semiárido. Las categorías Cordillera, Nieves eternas y Glaciar presentarían caudales medios altos a finales de primavera $\mathrm{y}$ principios de verano. No existe la combinación de las categorías Subhúmedo y Lagos.

Para las categorías Húmedo y Muy húmedo, se esperan caudales medios anuales mayores. La presencia de categorías pluviales se relaciona con caudales continuos y un aumento importante en los caudales punta. La categoría Precordillera y la categoría Cordillera presentarían hidrogramas bimodales; en el caso de Precordillera, el caudal punta mayor se produciría en invierno por los aportes pluviales, y lo contrario en la categoría Cordillera, debido al aporte del derretimiento de nieves en verano. Las categorías Nieves eternas y Glaciar se comportarían de forma similar que la categoría Cordillera. La categoría Lagos, produce un amortiguamiento de las variaciones estacionales del hidrograma así como un desfase temporal de estos caudales; se espera que éstos ocurran al final de las estaciones invernales y estivales. No existe la combinación de las categorías Muy húmedo y Nieves eternas.

En la Tabla 2.2 se muestran los códigos REC-Chile, en un segundo nivel de clasificación, para cuatro estaciones de aforo distribuidas a lo largo del país. A partir de estos códigos se realiza una interpretación descriptiva de su hidrograma medio anual. 
CAPÍTULO 2 IMPLEMENTACIÓN REC-CHILE

Tabla 2.2. Estaciones de aforo y sus correspondientes códigos REC-Chile usadas para interpretar el patrón hidrológico.

\begin{tabular}{|c|c|c|c|c|}
\hline Estaciones de aforo & Cuenca & $\begin{array}{c}\text { Q50 } \\
\left(\mathrm{m}^{3} / \mathrm{s}\right)\end{array}$ & $\begin{array}{l}\text { Código } \\
\text { REC- } \\
\text { Chile }\end{array}$ & $\begin{array}{c}\text { Clase en un } \\
2^{\circ} \text { nivel } \\
\text { REC-Chile }\end{array}$ \\
\hline $\begin{array}{l}\text { Río Salado antes } \\
\text { confluencia río Curti. }\end{array}$ & Loa & 0.094 & 154133 & $\mathrm{Ar} / \mathrm{Es}$ \\
\hline $\begin{array}{l}\text { Río Choapa en } \\
\text { Salamanca. }\end{array}$ & Choapa & 4.930 & 345223 & $\mathrm{Mw} / \mathrm{M}$ \\
\hline $\begin{array}{l}\text { Río Aconcagua en } \\
\text { Chacabuquito. }\end{array}$ & Aconcagua & 28.038 & 485321 & $\mathrm{~W} / \mathrm{G}$ \\
\hline Río Itata en Coelemu. & Itata & 310.405 & 535453 & Vw/Mm \\
\hline
\end{tabular}

La estación Río Salado pertenece a la superposición de las categorías Árido y Nieves eternas (Ar/Es). Para esta clase se espera que el patrón hidrológico presente bajos caudales medios anuales, caudales medios mensuales máximos en la época de derretimiento de nieves (enero a marzo) y caudales medios mensuales mínimos en época fría (junio a agosto.). La estación Río Choapa pertenece a la superposición de las categorías Subhúmedo y Cordillera $(\mathrm{Mw} / \mathrm{M})$, se espera que esta estación tenga caudales medios anuales mayores que la estación Río Salado y que el hidrograma presente dos caudales punta, uno al comienzo de la época de derretimiento de nieves (noviembre-diciembre) y otro más pequeño en la época de lluvias (junioagosto). Por último, la estación Aconcagua tiene categorías Húmedo y Glaciar (W/G), el caudal medio anual debería ser mayor que la estación anterior, el hidrograma debiera tener un caudal medio mensual máximo al comienzo del verano (diciembre-enero) y caudales relativamente bajos el resto del año. Finalmente, la estación Itata tiene categorías Muy húmedo y Precordillera $(\mathrm{Vw} / \mathrm{Mm})$, por lo tanto, el caudal medio anual será mayor que el caudal de todas las anteriores, los caudales medios mensuales máximos serán en la época de lluvias (junio a agosto) y caudales medios mensuales mínimos en la época de verano (enero a marzo) 
La Tabla 2.2, muestra que los caudales medios son coherentes con la clasificación, así como con los hidrogramas anuales (Fig. 2.3). Para todas las estaciones, la forma del hidrograma es similar a la descrita por las clases de la REC-Chile, así como también sus variaciones estacionales.

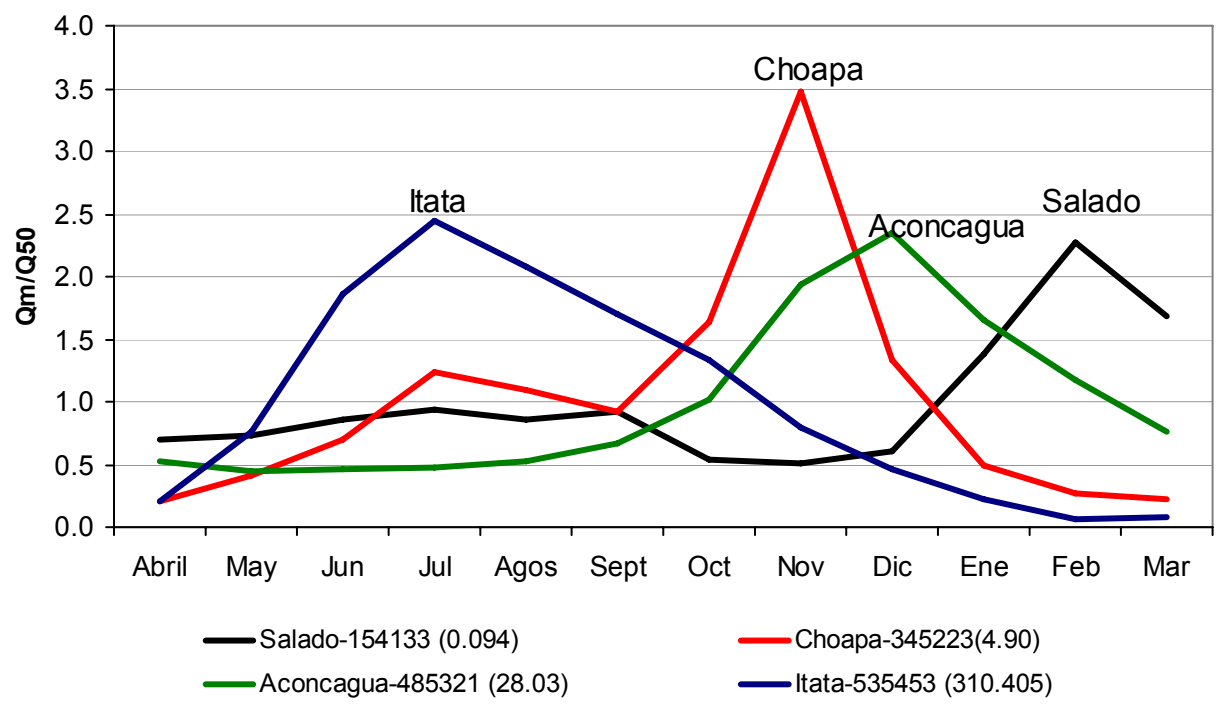

Figura 2.3. Hidrograma medio anual de 4 estaciones de aforo asociadas a 4 códigos REC-Chile. Para interpretación del código REC-Chile ver Tabla 2.1. En el eje Y corresponde a la división entre el caudal medio mensual con el caudal con un prob de excedencia igual al 50\%. La asociación del código REC-Chile con la estación de aforo correspondiente ver Tabla 2.2. Entre paréntesis se muestra el caudal medio anual para cada estación.

Para predecir la calidad geoquímica natural de las aguas debe ser considerada la litología local, el clima, la vegetación y el tiempo de residencia del agua en el acuífero, el cual resulta ser una función del clima (Drever 2002). Otro aspecto importante a considerar es la composición de las aguas que entra al sistema (Allan \& Castillo 2007) y el origen de las 
CAPÍTULO 2 IMPLEMENTACIÓN REC-CHILE

aguas circulantes en el río. Los factores controladores utilizados para predecir la calidad geoquímica natural de las aguas son Clima, Origen de Flujo y Geología, por lo tanto, el tercer nivel de clasificación permite realizar una predicción del valor medio de Conductividad Eléctrica (CE, $\mu \mathrm{S} / \mathrm{cm})$ para cada clase.

Se espera que la clase Árido del factor Clima tenga altos valores de CE, y a medida que aumenta el nivel de precipitación, CE disminuya; por tanto, la categoría Muy húmedo debería tener la CE más baja. Las categorías del segundo factor controlador, Origen de Flujo, afectan en menor medida la $\mathrm{CE}$ que el factor Clima. Se espera que las categorías de origen pluvial (Llano, Valle y Precordillera) presenten menor CE que aquellas provenientes del derretimiento de nieves (Cordillera, Nieves eternas y Glaciar) producto de la lixiviación de los terrenos volcánicos. La categoría Lagos puede presentar menores valores por los procesos físicoquímicos producidos en los lagos. Por ultimo, se espera que la categoría del tercer factor (Geología) presente valores de CE de acuerdo a la presencia de iones asociada a cada tipo de litología. Así, la categoría Depósito no consolidado presentaría valores bajos de CE, seguida por la categoría rocas Plutónicas, rocas Volcánicas, rocas Sedimentarias con mezcla de volcánicas, rocas sedimentarias y por rocas Carbonatadas. Un caso especial es la categoría rocas Volcánicas fracturadas, ya que éstas permiten una mayor lixiviación de los iones.

En la Figura 2.4 se muestran los valores de $\mathrm{CE}$ de puntos de muestreo agrupados según el código REC-Chile a un tercer nivel. Estos valores parecen estar en concordancia con las interpretaciones realizadas a partir de 
CAPÍTULO 2 IMPLEMENTACIÓN REC-CHILE las clases REC-Chile. Se observa que las clases con una categoría Árido (Ar/Es/Mx y $\mathrm{Ar} / \mathrm{Es} / \mathrm{Vf})$ presentan los valores mayores y que éstos disminuyen a medida que aumenta el nivel de precipitación. Se observa también el efecto del factor Origen de Flujo, ya que en la categoría Muy húmedo combinada con la categoría de Glaciar $(\mathrm{Vw} / \mathrm{G} / \mathrm{Mx})$ presenta la mayor concentración, mientras que la categoría Lagos $(\mathrm{Vw} / \mathrm{La} / \mathrm{Mx})$ tiene la menor concentración de CE. Por último, dentro de la clase Ar/Es se aprecia que la categoría rocas Volcánicas fracturadas (Ar/Es/Vf) tiene una mayor concentración que las rocas compuestas de origen volcánico y sedimentario (Ar/Es/Mx).

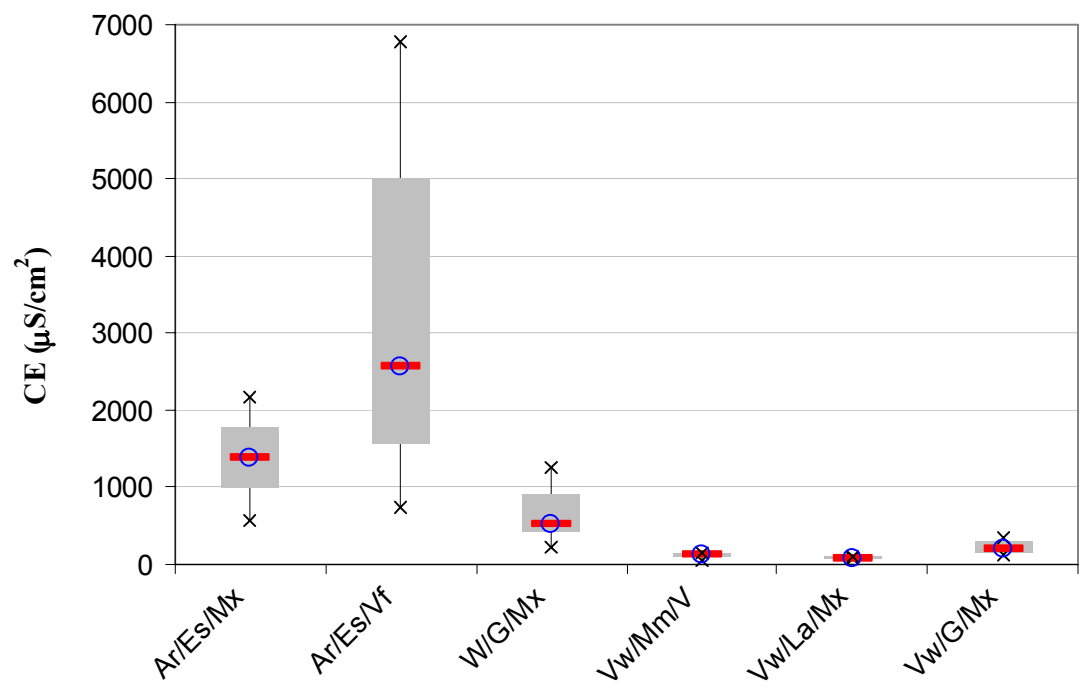

Figura 2.4. Valores medios, percentiles 25\% y 75\% y valores máximos y mínimos de Conductividad Eléctrica $(\mu \mathrm{S} / \mathrm{cm}$.$) de 6$ clases de REC-Chile. La codificación en el eje $\mathrm{X}$ representa la anotación simplificada de las clases del tercer nivel de clasificación REC-Chile. Mayores detalles ver Tabla 2.1 


\subsection{DISCUSIÓN}

Los resultados de este estudio muestran que es posible llevar a cabo la implementación de la REC a las condiciones ambientales de Chile. Los factores controladores y categorías desarrolladas para este país parecen recoger de una forma adecuada las diversas condiciones climáticas, hidrológicas y geológicas. Por ejemplo, utilizando la REC-Chile a un segundo nivel, es decir, combinando los factores Clima y Origen de Flujo resulta posible describir aproximadamente el patrón hidrológico de un tramo de río. Esto permite comparar magnitudes de caudales medios anuales y determinar la forma básica del hidrograma anual de un tramo de río, como se ha mostrado en el presente estudio.

Además, en un tercer nivel de clasificación se logró una buena discriminación de la calidad geoquímica del agua. Esto concuerda con estudios previos en los cuales se señalan que la precipitación, la litología en la cuenca, el origen del flujo y el tiempo de residencia son los factores que más influyen en la calidad geoquímica de las aguas (Allan \& Castillo 2007, Drever 2002, Custodio \& Llamas 1976).

Es importante señalar que la REC-Chile tiene incorporado el concepto de river continuum (Vannote 1980), manifestando los gradientes longitudinales hacia aguas abajo como resultado de la propagación de las características de las categorías.

El desarrollo de la REC-Chile en un ambiente SIG permite una fácil visualización y manejo de los datos. Una gran ventaja de esta herramienta es 
CAPITULO 2 IMPLEMENTACIÓN REC-CHILE la posibilidad de gestionar información proveniente de los factores controladores que el usuario seleccione de acuerdo con los objetivos y escala de trabajo. Esto permite una gran flexibilidad para obtener patrones de distribución de variables hidrológicas y fisicoquímicas que determinan las características ecológicas de los tramos de río.

Una gestión coordinada entre la REC-Chile y una base de datos georreferenciada de comunidades acuáticas, por ejemplo macroinvertebrados o peces, trae grandes beneficios para ser utilizada en la gestión y planificación de ecosistemas acuáticos. Entre estos permitiría determinar patrones de distribución espacial de especies con escasa información (Peredo-Parada et al. 2009, Snelder et al. 2004), diseñar redes de muestreo asegurando una adecuada distribución de puntos de muestreo sobre el área de interés (Hawkins et al. 2000) o patrones de idoneidad del hábitat a nivel de mesoescala.

Otra aplicación de importancia de la REC-Chile es que permite definir tramos de río que mantengan condiciones naturales, pudiendo ser usados como tramos de referencia para planes de conservación o restauración (Hawkins et al. 2000). En caso que no existan tramos en condiciones naturales, la REC-Chile permite determinar el máximo potencial ecológico existente en tramos pertenecientes a una misma clase utilizando diversos índices, los cuales pueden ser ícticos (Noble et al. 2007, Schmutz et al. 2007), índices de macroinvertebrados (Figueroa et al. 2007, 2003), índices de vegetación ripariana (Palma et al. 2009, Munné et al. 2003, 1998). Como una consecuencia de la utilización de estos índices en la REC-Chile es posible determinar el nivel de alteración, ya sea hidrológica o ecológica, de 
CAPÍTULO 2 IMPLEMENTACIÓN REC-CHILE

los tramos de río con respecto al tramo seleccionado como referencia. También puede emplearse para extrapolar información ecológica en las clases del REC-Chile (Omernik \& Bailey 1997). Estas aplicaciones aportan una gran utilidad en la priorización y diseño de actividades de restauración fluvial. 


\subsection{CONCLUSIONES}

La clasificación REC-Chile es una adaptación a las condiciones climáticas, morfológicas y geológicas de Chile de la River Environmental Classification (REC) desarrollada por Snelder \& Biggs (2002).

Esta clasificación es una herramienta que clasifica mediante una codificación a los tramos de río de acuerdo a una superposición jerárquica de 6 factores que controlan el patrón hídrico. (Clima, Origen de Flujo, Geología, Posición Relativa de Subsubcuenca y Pendiente del tramo). La clasificación de los tramos de río, se representa como un código de 6 dígitos, en donde la posición del dígito es el nivel clasificación y el valor de éste es la categoría del factor en ese nivel.

Las ventajas de la REC-Chile son variadas. En primer lugar permite describir diversos patrones ecohidrológicos de los ríos de Chile mediante la superposición de factores controladores adecuados según el objetivo de clasificación, permitiendo así, el estudio a diversas escalas espaciales. Además, posee la capacidad de manejar a los ríos como una red hídrica considerando la continuidad longitudinal, permitiendo detectar cambios de una subcuenca a otra aunque ésta pueda estar geográficamente cerca, logrando así una mejor caracterización de los ríos de Chile. Está implementada en un ambiente SIG lo que facilita su interpretación geográfica y el cambio a distintas escalas de trabajo.

Una rápida interpretación de las clases de la REC-Chile a distintos niveles de clasificación, permite realizar una sencilla descripción aproximada de los 
CAPÍTULO 2 IMPLEMENTACIÓN REC-CHILE

diversos patrones fluviales, por ejemplo, a un segundo nivel de clasificación es posible describir el hidrograma medio anual de los tramos de ríos. A un tercer nivel de clasificación es posible comparar aspectos de la calidad geoquímica natural de las aguas.

Finalmente esperamos que la clasificación REC-Chile se constituya en una herramienta para la conservación y gestión de los ecosistemas fluviales en Chile, apoyando en la determinación del estado ecológico potencial, en la selección de métodos idóneos para asignar la metodología óptima para determinar caudales ecológicos para cada tipo de río y en los tramos de referencia para una restauración fluvial. 


\subsection{BIBLIOGRAFIA}

ALLAN D \& M CASTILLO. 2007. Stream ecology. Structure and function of running waters. $2^{\text {nd }}$ edition. Springer Dordrecht, The Netherlands. 436 pp.

BAEZA D, F MARTÍNEZ-CAPEL \& D GARCÍA DE JALÓN. 2003. Variabilidad temporal de caudales: Aplicación a la gestión de ríos regulados. Ingeniería del agua 10 (4): 469-478.

BAHAMONDE N, A CARVACHO, C JARA, M LÓPEZ, F PONCE, MA RETAMAL \& E RUDOLFF. 1998. Categorías de conservación de decápodos nativos en aguas continentales de Chile. Boletín del Museo Nacional de Historia Natural 47:91-100.

BAILEY RG, RD PFISTER \& JA HENDERSON. 1978. Nature of Land and Resource Classification. Journal of Forestry 76 (10):650-655

CAMOUSSEIGHT A. 2006. Estado de conocimiento de los Ephemeroptera de Chile. Gayana 70(1): 50-56.

CONAMA 2007. Estrategia Nacional de Gestión Integrada de Cuencas Hidrográficas. Gobierno de Chile. 60 pp.

CUSTODIO E \& M LLAMAS. 1976. Hidrología Subterránea. Omega S.A. ed. Barcelona. 1157pp.

DREVER J. 2002. The Geochemistry of Natural Waters. Surface and Groundwater Environments. Prentice Hall. Third Edition. New Jersey. ISBN: 0-13-272790-0. $436 \mathrm{pp}$.

DYER B. 2000. Sistematic review and biogeography of the freshwater fishes of Chile. Estudios Oceanológicos 19: 77-98.

FIGUEROA R, C VALDOVINOS, E ARAYA \& O PARRA. 2003. Macroinvertebrados bentónicos como indicadores de calidad de agua de ríos del sur de Chile. Revista Chilena de Historia Natural 76: 275-285.

FIGUEROA R, A PALMA V RUIZ \& X NIELL. 2007. Análisis comparativo de índices bióticos utilizados en la evaluación de la calidad de las aguas en un río mediterráneo de Chile: Río Chillán, VIII Región. Revista Chilena de Historia Natural 80: 225-242. 
FLEMING S. 2005. Comparative analysis of glacial and nival streamflow regimes with implications for lotic habitat quantity and fish species richness. River research and applications. 21: 363-379.

FRISSELL C, WJ LISS, CE WARREN \& MD HURLEY. 1986. A hierarchical framework for Stream Habitat Classification: Viewing Streams in Watershed context. Environmental Management 10: 199-214.

GONZÁlEZ L, M MARDONES, A SILVA \& E CAMPOS. 1999. Hidrogeoquímica y comportamiento del agua subterránea en la cuenca del río Claro, Región del Biobío, Chile. Revista geológica de Chile. 26 (2): 145-157.

GONZÁLEZ DEL TÁNAGO M \& D GARCÍA DE JALÓN. 2006. Propuesta de caracterización jerárquica de los ríos españoles para su clasificación según la Directiva Marco de la Unión Europea. Limnética 25 (3): 693-712.

HABIT E, B DYER \& I VILA. 2006. Estado de conocimiento de los peces dulceacuícolas de Chile. Gayana 70 (11): 100-112.

HARRIS N, A GURNELL, D HANNAH \& G PETTS. 2000. Classificaction of rivers regimes: a context for hydroecology. Hydrological Process. 14: 2831-2848.

HAWKINS C, R NORRIS, J GERRITSEN, R HUGHES, S JACKSON, R JOHNSON \& J STEVENSON. 2000. Evaluation of the use of landscape classifications for the prediction of freshwater biota: synthesis and recommendations. Journal of American Benthological Society. 19 (3): 541-556.

HERAS R 1973. Ediciones Centros de Estudios Hidrográficos, Madrid. 46 pp.

HERRERA C, JJ PUEYO, A SAEZ, \& B VALERO-GARCÉS. 2006. Relación de aguas superficiales y subterráneas en el área del lago Chungará y lagunas de Cotacotani, norte de Chile: un estudio isotópico. Revista geológica de Chile. 33 (2): 299-325.

JARA CG, EH RUDOLPH \& ER RIQUELME. 2006. Estado de conocimiento de los malacostracos dulceacuícolas de Chile. Gayana 70(1): 40-49.

JOHNSON L, C RICHARDS, G HOST \& J ARTHUR. 1997. Landscape influences on water chemistry in Midwestern stream ecosystems. Freshwater Biology 37 (1): 193-208. 
CAPÍTULO 2 IMPLEMENTACIÓN REC-CHILE MCDOWALL MC. 2000. Biogeography of the southern cool-temperate galaxioid fishes: evidence from metazoan macroparasite faunas. Journal of Biogeography 27: 1221-1229.

MONTGOMERY DR \& JM BUFFINGTON. 1997. Channel-reach morphology in mountain drainage basins. Geological Society of America Bulletin 109: 596-611.

MUNNÉ A, C SOLA \& N PRAT. 1998. QBR: Un índice rápido para la evaluación de la calidad de los ecosistemas de ribera. Tecnología del Agua 175: 20-37.

MUNNÉ A, N PRAT, C SOLA, N BONADA \& M RIERADEVALL. 2003. A simple field method for assessing the ecological quality of riparian habitat in rivers and streams. QBR index. Aquatic Conservation: Marine and Freshwater Ecosystems 13: 147-164.

OMERNIK J \& R BAILEY. 1997. Distinguish between watersheds and ecoregions. Journal of the American Water Resources Association. 33 (5): 935949.

ORTÍZ JC \& H DÍAZ-PAEZ. 2006. Estado de conocimiento de los anfibios de Chile. Gayana 70(1): 114-121.

PALMA A, R FIGUEROA \& VH RUIZ. 2009. Evaluación de ribera y hábitat fluvial a través de los índices QBR e IHF. Gayana. 73(1): 57-63.

PARADA E \& S PEREDO. 2005. La relocalización como una herramienta de conservación y manejo de la biodiversidad. Lecciones aprendidas con Diplodon chilensis (Gray 1828) (Bivalvia:Hyriidae). Gayana 69(1): 41-47.

PARADA E \& S PEREDO. 2006. Estado de conocimiento de los bivalvos dulceacuícolas de Chile. Gayana 70(1): 82-87.

PEREDO S, E PARADA, I VALDEBENITO \& M PEREDO. 2005. Relocation of the freshwater mussel Diplodon chilensis (Hyriidae) as a strategy for its conservation and management. Journal Molluscan Studies 71: 195-198.

PEREDO S, E PARADA, I VALDEBENITO \& M PEREDO. 2006. Effect of aerial exposure on Physiological condition and survival of Diplodon chilensis (Hyriidae) during translocation. Malacologia 49(1): 217-221.

PEREDO-PARADA M, F MARTÍNEZ-CAPEL, V GARÓFANO-GÓMEZ, M ATENAS \& F RIESTRA.2009. Base de datos eco-hidrológica de los ríos de chile: una herramienta de gestión para los ecosistemas acuáticos. Gayana 73 (1): 119129. 
CAPÍTULO 2 IMPLEMENTACIÓN REC-CHILE

PEREDO-PARADA M, F MARTÍNEZ-CAPEL, JD ALCARAZ \& M BARGAY. 2009. Potential geographical distribution of two endemic freshwater fishes $(P$. gillissi and D. camposensis) of Chile. En: Int. Association of Hydraulic Engineering and Research (IAHR) and Universidad de Concepción (Ed). Proceedings of the International Symposium on Ecohydraulics. ISBN: 978-981-082100. Concepción. Chile

PÉREZ-LOSADA M, CG JARA, G BOND-BUCKUP \& KA CRANDALL. 2002. Conservation phylogenetics of Chilean freshwater crabs Aegla (Anomura, Aeglidae): assigning priorities for aquatic habitat protection. Biological Conservation 105: 345-353.

RUTLLANT J. 2004. Aspectos de la circulación atmosférica de gran escala asociada al ciclo ENOS 1997-1999 y sus consecuencias en el régimen de precipitación en Chile central. Avaria S. Carrasco J. Rutllant J. and Yañez E. (eds). El niño-La niña 1997-2000 sus efectos en Chile. CONA, Chile, Valparaíso 61-76.

SLIVA L \& D DUDLEY. 2001. Buffer Zone versus Whole Catchment Approaches to Studying Land Use Impact on River Water Quality. Water Research 35 (14): 3462-3472.

SNELDER T \& B BIGGS. 2002. Multiscale River Enviroment Classification for Water Resources Management. American Water Resources Association 38 (5): 1225-1239.

SNELDER T, B BIGGS \& M WEATHERHEAD. 2004a. New Zealand River Enviroment Classification. User Guide. Ministry for the Environment. Wellington, New Zealand.

SNELDER T, F CATTANEO, A SUREN \& B BIGGS. 2004b. Is the River Environment Classification an improved landscape-scale classification of rivers? Journal of the North American Benthological Society. 23 (3): 580-589.

SNELDER T, B BIGGS \& R WOODS. 2005. Improved Eco-Hydrological Classificaction of Rivers. River Research and Applications. 21: 609-628.

VALDOVINOS C. 2006. Estado de conocimiento de los gastrópodos dulceacuícolas de Chile. Gayana 70(1): 88-95.

VANNOTE RL, GW MINSAHLL, KW CUMMINS, JR SEDELL \& CE CUSHING. 1980. The River Continuum Concept. Canadian Journal of Fisheries and Aquatic Science. 37: 130-137.

VERA A \& A CAMOUSSEIGHT. 2006. Estado de conocimiento de los plecópteros de Chile. Gayana 70(1): 57-64. 
CAPÍTULO 2 IMPLEMENTACIÓN REC-CHILE VILA I \& X MOLINA. 2006. Capítulo 3: Variables químicas y biológicas en Manual de Calidad del Agua. Servicio Agrícola y Ganadero. Eds. Molina y Vila. 106 pp.

VILA I, R PARDO, B DYER \& E HABIT. 2006. Peces límnicos: Diversidad, origen y estado de conservación. En: Vila I, A Veloso, R Schlater \& C Ramirez (eds.) Macrófitas y vertebrados de los sistemas límnicos de Chile: 73-103. Editorial Universitaria, Stgo., Chile.

VILLAGRAN C \& LF HINOJOSA. 2005. Esquema biogeográfico de Chile. 2005, Capítulo 33: 551-577 EN: "Regionalización Biogeográfica en Iberoámeríca y tópicos afines" (Jorge Llorente Ousquets \& Juan J. Morrone, Editores). Ediciones de la Universidad Nacional Autónoma de México, Jiménez Editores, México, 577 pp.

WINTERBOUR MJ, JS ROUNICK \& B COWIE. 1981. Are New Zealand stream ecosystems really different. New Zealand Journal of Marine and Freshwater Research 15: 321-328. 

Capitula 3

¿Es la REC-Chile una adecuada clasificación para los ríos de Chile? 



\subsection{RESUMEN}

La clasificación de los ríos es un paso importante para poder entender los distintos procesos fluviales. Existen diversos tipos de clasificación, entre los cuales destacan las clasificaciones desarrolladas a partir de la deducción de factores controladores de los procesos fluviales, también denominadas como clasificaciones a priori, y aquellas clasificaciones desarrolladas a partir de datos, denominadas clasificaciones a posteriori. Entre las ventajas que presentan las clasificaciones a priori se encuentran la factibilidad de implementarlas en zonas con poca información, la interpretación de sus clases y la posibilidad de extrapolar su información a zonas desprovistas de ésta. Debido a su facilidad en la implementación las clasificaciones a priori son altamente utilizadas, sin embargo no todas son sometidas a una evaluación que permita determinar el grado de ajuste entre las clases y la variabilidad de los datos.

La REC-Chile es una clasificación a priori implementada en los ríos de Chile, la cual está basada en la superposición jerárquica de los factores controladores Clima, Origen de Flujo, Geología, Posición Relativa de Subsubcuenca, Uso de Suelo y Pendiente del tramo. Esta clasificación se sometió a evaluación bajo los objetivos de discriminación del patrón hidrológico y la calidad geoquímica de las aguas mediante una variación del test de similaridad de Van Sickle (1997) y posteriormente se realizó una interpretación de las clases para ambos objetivos de clasificación. La interpretación de las clases se llevó a cabo mediante Análisis de 
Correspondencia para el patrón hidrológico y mediante gráficos de cajas y bigotes para la calidad geoquímica.

Los resultados del test de clasificación para ambos objetivos mostraron resultados satisfactorios mostrando que la REC-Chile es una clasificación que discrimina espacialmente los procesos fluviales de los ríos de Chile. Como resultado de estos análisis es posible realizar una interpretación descriptiva del hidrograma en régimen natural de los tramos de ríos, así como valores medios de la conductividad eléctrica.

Dada la bondad del resultado de la evaluación de clasificación, la posibilidad de interpretación de sus clases y por las características propias de la REC-Chile como una clasificación a priori, se espera que ésta se convierta en una plataforma para la gestión y planificación de los ríos de Chile y de sus ecosistemas. 


\subsection{INTRODUCCIÓN}

La acción de clasificar los ríos tiene un rol fundamental para interpretar los procesos que explican la distribución de los distintos tipos de funcionamiento ecológico en sus respectivas cuencas vertientes (Naiman 1998). Las clasificaciones son desarrolladas por diversos motivos, entre los más comunes se encuentran proveer un marco espacial para estimar el estado de un ecosistema, para la investigación, inventarios, monitorización y gestión (Snelder et al. 2005, Omernik \& Bailey 1997). Para que una clasificación sea útil en la gestión de cuencas, ésta debe poseer la particularidad de poder extrapolar espacialmente la información a cuencas desprovistas de ésta (Snelder et al. 2009, Omernik \& Bailey 1997).

Con respecto a las clasificaciones hidrológicas, González del Tánago \& García de Jalón (2006) distinguen clasificaciones basadas en los diferentes métodos descritos por Bailey et al. (1978). Estos autores definen las clasificaciones taxonómicas y aquellas basadas en una regionalización. Las taxonómicas son construidas a partir de una serie de atributos como variables biológicas, hidrológicas, etc. Las regionalizaciones responden a un enfoque físico en donde se van determinando las diferencias observadas a diferentes escalas espaciales.

Una definición similar se contempla en la Directiva Marco del Agua de la Unión Europea (Comisión Europea 2000) en donde se define el Sistema A de clasificación, basado en enmarcar al río en su entorno biogeográfico, permitiendo establecer una tipología regional a nivel de subcuenca, y el 
Capítulo 3 EVAluación de LA REC-Chile

sistema $\mathrm{B}$, en el que se propone considerar una serie de factores de diversas escalas espaciales para la caracterización de los ríos.

Otros autores, como Snelder et al. (2005), definen dos tipos de clasificaciones según el método de construcción de éstas. Así separan las clasificaciones a priori (enfoque de arriba abajo, top-down) en las cuales las regiones ecológicas son definidas en forma deductiva basada en los factores ambientales que influencian los patrones hidrológicos de un río. Por otro lado están las clasificaciones a posteriori (enfoque de abajo a arriba, downtop), realizadas empíricamente mediante métodos estadísticos usando datos hidrológicos.

Las ventajas de una clasificación a priori es que permite su implementación en zonas con poca información (Peredo-Parada et al. 2010); siendo ésta una de las grandes limitaciones de Chile y en general de los países en vías de desarrollo.

Dentro de las clasificaciones tipo a priori, las más comunes son las regionalizaciones (ecoregiones) y aquellas basadas en factores controladores (Snelder \& Biggs 2002). Las ecorregiones se basan en comprender los patrones de los ecosistemas a gran escala, para desarrollar mosaicos en los cuales las subcuencas pueden considerarse como homogéneas para ciertas características (Omernik 1987). En cambio, las basadas en factores controladores clasifican el territorio mediante unidades generales del paisaje, organizadas jerárquicamente a distintas escalas, basándose en una visión conceptual de cómo están organizados los ecosistemas acuáticos (Snelder \& Biggs 2002). 
La diferencia entre ecorregiones y clasificaciones basadas en factores controladores radica principalmente en la definición de las fronteras de sus clases. Las ecorregiones pueden ser consideradas como geográfica dependientes entre sí, mientras que las de factores controladores son geográficamente independientes (sensu Detenbeck et al. 2000). Esto quiere decir que las ecorregiones tienen las fronteras fijas y definidas para una única escala espacial. Mientras que las clases (a varias escalas) basadas en factores controladores son delimitadas usando características climáticas y morfológicas, asumiendo que éstas controlan los procesos de la cuenca a distintas escalas espaciales de forma jerarquizada (Snelder \& Biggs 2002). Esta configuración de las clases, más la superposición jerárquica de los factores controladores, permite trabajar a diferentes escalas espaciales (Snelder et al. 2005).

El éxito de este tipo de clasificaciones en la discriminación de los patrones hidrológicos está determinado por la correcta elección de los factores controladores y su división en categorías adecuadas. Además, el hecho de considerar los factores controladores de modo jerárquico, permite aplicar sobre estas clasificaciones modelos biológicos predictivos más precisos (Hawkins et al. 2000), por ejemplo para la distribución de peces en una cuenca (Hopkins II \& Burr 2009). Sin embargo, no es común que las clasificaciones a priori sean sometidas a una evaluación estadística para determinar el grado de ajuste de las clases a la variabilidad de los datos.

En Chile se ha desarrollado una Clasificación Eco-Hidrológica de los ríos (REC-Chile) (Peredo-Parada et al. 2010). Esta clasificación es del tipo $a$ 
Capítulo 3 Evaluación de LA REC-Chile

priori, construida a partir de la superposición jerárquica de 6 factores controladores (Clima, Origen de flujo, Geología, Posición relativa de subsubcuenca y Pendiente del tramo). Se basa en que el clima, la topografía, la geología y la cobertura del uso de suelo son los principales causantes de la variación del patrón hidrológico en Chile.

Este capítulo tiene como objetivo evaluar si la REC-Chile es una adecuada clasificación para los ríos de Chile bajo diversos objetivos de clasificación, así como interpretar las clases resultantes mediante correspondencias con variables descriptoras del hidrograma anual del río, en condiciones medias del flujo. 


\subsection{METODOLOGÍA}

Para comprobar si los factores controladores definidos, así como sus categorías y reglas de asignación, han sido determinados de forma correcta se realizaron dos evaluaciones de la clasificación REC-Chile, la primera bajo un objetivo hidrológico y la segunda bajo un objetivo geoquímico. En ambas evaluaciones se usó una variación de la metodología propuesta por Van Sickle (1997) para determinar si los datos asociados a las clases presentan una estructura de clasificación. La interpretación hidrológica de las clases se realizó mediante dos Análisis de Correspondencias (AC) entre las clases de la REC-Chile (a diferentes niveles jerárquicos) y las variables del hidrograma medio anual de estaciones de aforo ubicados a lo largo de Chile. La interpretación geoquímica se realizó mediante diagramas de cajas y bigotes entre las clases REC-Chile y valores de la conductividad eléctrica en tramos de río distribuidos a lo largo de Chile.

\subsubsection{Evaluación hidrológica de la REC-Chile}

Para lograr una adecuada discriminación de la variación espacial del patrón hidrológico se consideraron los factores Clima, Origen de Flujo y Posición Relativa de subsubcuenca (PRScuenca), definiendo esquemas de clasificación según su combinación jerárquica. Estos esquemas y la metodología utilizada se detallan a continuación. 
3.3.2. Fuentes de datos y variables del hidrograma

Las series temporales de caudales medios mensuales fueron suministradas por la Dirección General de Aguas de Chile (DGA). Se eligieron 48 estaciones de aforo distribuidas a lo largo de toda la clasificación RECChile que tuviesen un régimen natural o levemente modificado (Figura 3.1). La longitud de las series de datos varía según la cada estación, con un promedio de 31 años (1971-2002) y un mínimo de 13 años (1989-2002).

De dichas series de datos se extrajeron variables hidrológicas de relevancia para las condiciones hidráulicas y ecológicas a gran escala, las cuales se pueden agrupar así: 1) magnitud media de caudal flujo; 2) magnitud media de caudales altos y bajos; 3) estacionalidad del caudal. Cada grupo de variables se determinó para tres escenarios hidrológicos: años medios $(50 \%$ probabilidad de excedencia), secos (10\%) y húmedos (95\%).

La magnitud del flujo se determinó mediante el caudal medio anual $\left(\mathrm{m}^{3} / \mathrm{s}\right)$ para los tres escenarios en análisis (Q50, Q10 y Q95, respectivamente). En años medios, se definieron las variables R50min y R50max; la primera de ellas (R50min) es el ratio entre el caudal medio mensual mínimo y Q50. Análogamente R50max es el ratio entre el caudal medio mensual máximo y Q50. De la misma forma, se definieron los ratios mínimos y máximos para años secos (R10min y R10max) y para años húmedos (R95min y R95max). 
CAPÍtUlo 3 EVALUACIÓN DE LA REC-CHILE

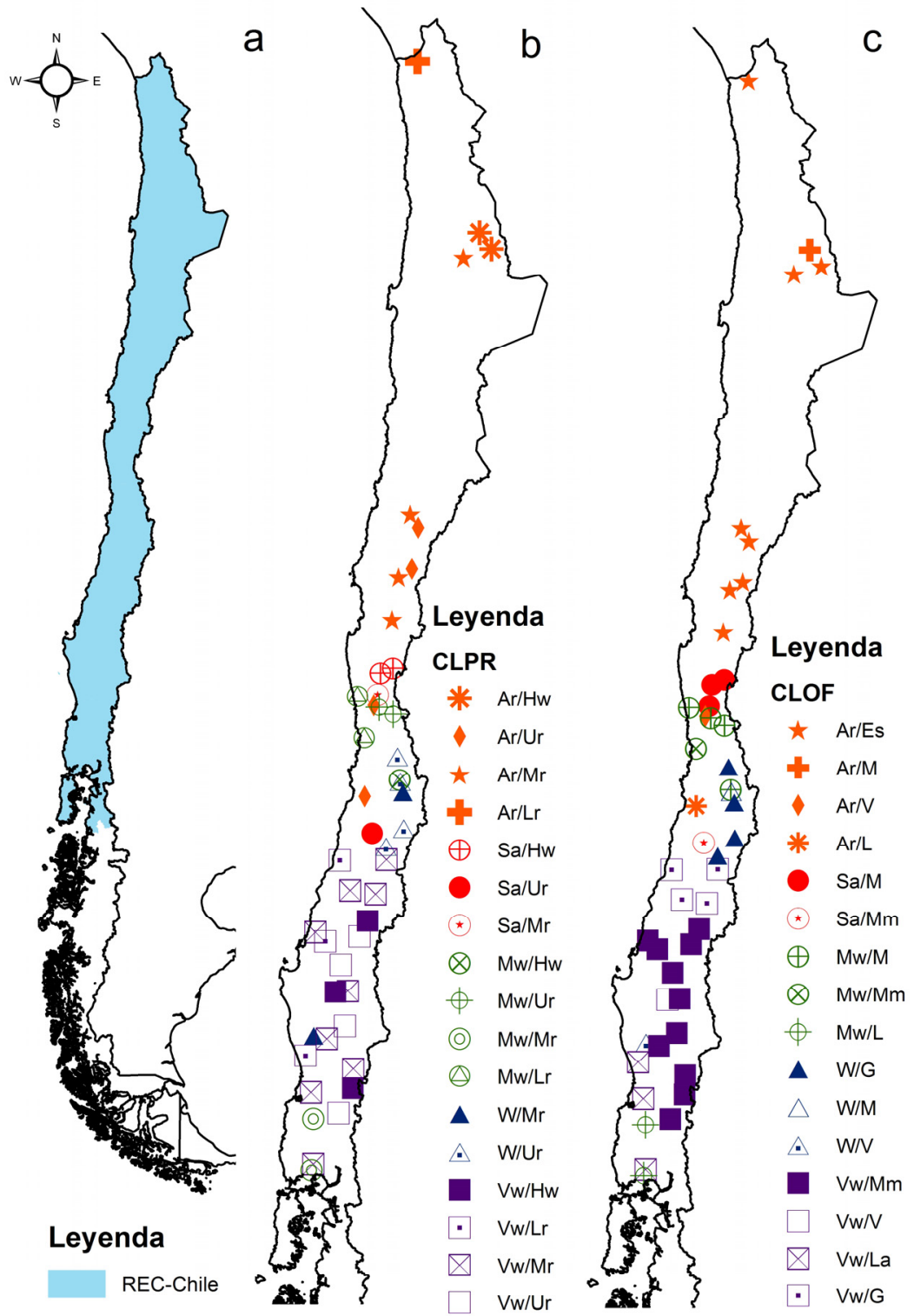

Figura 3.1. Mapa de Chile en donde se muestra la zona geográfica donde fue implementada la REC-Chile (fig. a). Se muestra la ubicación de las estaciones de aforo y su clasificación según los esquemas desarrollados mediante la superposición de los factores Clima y Posición Relativa de la Subsubcuenca (CLPR) (fig. b) y el esquema desarrollado a partir de los factores Clima y Origen de Flujo (CLOF) (fig. c). La interpretación de las clases asociadas a cada esquema se incluyen en la Tabla 2.1 
La estacionalidad del flujo se determinó mediante dos variables, la primera es el mes al cual ocurre el caudal medio mensual mínimo (Tmin) y la segunda es el mes al cual ocurre el caudal medio mensual máximo (Tmax). Estas dos variables se determinaron para los tres escenarios hidrológicos, resultando las variables T50min y T50max para años medios, T10min y T10max para años secos y T95min y T95max para años húmedos. Los meses fueron asociados a números de acuerdo a un año natural, es decir, al primer mes del año (enero) se le asignó el valor 1, a febrero se le asignó el valor 2 y sucesivamente hasta diciembre como el mes número 12.

\subsubsection{Test de Clasificación hidrológica}

Un test de clasificación consiste en cuantificar el ajuste de la clasificación a la variabilidad de los objetos descritos por múltiples variables (Van Sickle 1997). Este test compara la disimilaridad media intra-clases con la disimilaridad media inter-clases, es decir, da una idea de lo compactas y aisladas que están las clases entre ellas.

El test de clasificación se realiza a partir de la matriz de disimilaridad obtenida por pares de puntos. De esta matriz se obtienen la disimilaridad media de los objetos en cada clase i $\left(\bar{W}_{i}\right)$ la disimilaridad media intra-clases $(\bar{W})$ y la media inter-clases $(\bar{B})$. Van Sickle (1997) propuso el índice M para cuantificar la robustez total de la clasificación $M=\bar{W} / \bar{B}$. Si no hay estructura de clase, entonces $\mathrm{M}=1(\bar{B}=\bar{W})$, y a medida que $\mathrm{M}$ disminuye, aumenta la estructura de clasificación (más distanciadas están las clases, con respecto a la distancia media entre ellas). La significación estadística del test 80 
CAṔ́tUlo 3 EVALUACIÓN DE LA REC-ChILE se determinó mediante un test de aleatoriedad $(n=1000 ; p<0.001)$ basado en la hipótesis nula de que "No hay estructura de clases" utilizando la técnica de remuestreo de bootstrap sin reemplazo. Este test, al utilizar distancias (disimilaridades) es el contrario de la implementación original de Van Sickle (1997) el cual usaba similaridades, sin embargo, los estadísticos y los p-values son equivalentes (para mayores detalles ver Snelder et al. 2005).

Una representación gráfica de la estructura de la clasificación se presenta mediante los dendrogramas de distancias medias (Van Sickle 1997, Van Sickle \& Hughes 2000). Éstos consisten en árboles cuyo tronco vertical está graficado a una distancia media inter-clases $(\bar{B})$ sobre el eje X. Cada clase de la clasificación se representa como una rama horizontal cuyo nodo terminal está a una distancia media intra-clases $\left(\bar{W}_{i}\right)$. La longitud de cada rama con respecto el tronco del árbol representa la diferencia entre disimilaridad media inter-clases y la disimilaridad de la clase i $\left(\bar{B}-\bar{W}_{i}\right)$. Cuanto mayor sea esta distancia (es decir, que las ramas sean más largas hacia la izquierda) más compactas y aisladas están las clases y por tanto, más robusta será la clasificación.

Para la evaluación de la REC-Chile se utilizaron dos grupos de variables de acuerdo a dos objetivos de clasificación. El primer objetivo está relacionado con la magnitud de los caudales en el hidrograma. Para ello se utilizaron dos esquemas de clasificación. El primer esquema (CL) se basó en el factor Clima; el segundo esquema (CLPR) corresponde a la combinación de los factores Clima y Posición Relativa de Subsubcuenca. El grupo de variables hidrológicas utilizadas para evaluar estos 2 esquemas fueron las variables de magnitud del flujo (Q50, Q10 y Q95) y las variables de magnitud media de 
CAPÍtUlo 3 EVAlUACIÓN DE LA REC-CHILE

flujos altos y bajos (R50max, R50min, R10max, R10min, R95max y R95min). Sus valores fueron asociados a las clases de los esquemas CL y CLPR, en cada tramo de río con estación de aforo $(\mathrm{N}=48)$. La distribución geográfica y la pertenencia de las estaciones de aforo al esquema CLPR se ilustra en la Figura 3.1b

El segundo objetivo de clasificación está relacionado con la estacionalidad del flujo. Se utilizó para la discriminación de la estacionalidad un tercer esquema de clasificación (CLOF), definido mediante la superposición de los factores Clima y Origen de flujo. El grupo de variables utilizadas para su evaluación fueron las de magnitud media de flujos altos y bajos (R50max, R50min, R10max, R10min, R95max, R95min) y las de estacionalidad propiamente dicha (T50min, T50max, T10min, T10max, T95min y T95max). Sus valores fueron asociados a las clases CLOF de cada tramo de río con estación de aforo $(\mathrm{N}=48)$. La distribución geográfica y la pertenencia de las estaciones de aforo al esquema CLOF se ilustra en la Figura 3.1c.

Se definen así tres esquemas para la evaluación de la REC-Chile; dos asociados a la magnitud del flujo (CL y CLPR) y uno a la estacionalidad del flujo (CLOF). Para cada esquema se ha determinado el índice M (Van Sickle 1997) de acuerdo al grupo de variables y sus correspondientes clases.

En los tres esquemas la matriz de disimilaridad se obtuvo mediante la distancia Bray-Curtis, eliminando del análisis aquellas clases que solo presentan un dato. Los cálculos de las disimilaridades $\bar{W}_{i}, \bar{W}, \bar{B}$, el análisis de permutaciones aleatorias, así como la determinación de los 
Capítulo 3 EVALuación de LA REC-Chile dendrogramas se realizaron mediante el paquete de funciones Env.Class desarrolladas por Snelder (comunicación. personal) para el programa estadístico R, versión 2.8.1 (R Development Core Team 2008).

Como complemento del test de evaluación de la REC-Chile, se desarrolló una clasificación a posteriori mediante un cluster jerárquico, con los mismos datos utilizados en el test de evaluación y el mismo número de clases de los esquemas REC-Chile. Este análisis permitió comparar una clasificación a priori (REC-Chile) con una clasificación a posteriori (utilizando la técnica de cluster jerárquico) desarrollada a partir de los datos hidrológicos, siendo utilizada como nivel de referencia en la evaluación de los tres esquemas de clasificación presentados a partir de la REC-Chile.

Además, la REC-Chile fue comparada con la Clasificación Hidrodinámica de Chile (CHID, Ferrando 1992-1993). Esta clasificación se basa en una división hidrodinámica del país a nivel de cuencas, en donde se consideran aspectos como el clima, el destino final del flujo (exorreísmo, endorreísmo, arreísmo), temporalidad del flujo (perenne, estacional, ocasional) y el origen de las aguas (Altiplánico, Andino, Pre-andino, Depresión central, Costero o Pampeano-Patagónico). De esta clasificación se obtuvieron dos esquemas de clasificación (CHID_CLPR y CHID_CLOF) las cuales fueron determinadas considerando los objetivos de clasificación e igual número de clases que los esquemas CLPR y CLOF respectivamente. Para cada una de estas dos clasificaciones CHID se determinó el índice M (Van Sickle 1997). Mediante este índice se compararon las clasificaciones CHID con sus homólogas REC-Chile, es decir, CHID_CLPR con el esquema CLPR y CHID_CLOF con el esquema CLOF. 


\subsubsection{Interpretación hidrológica de las clases}

Para la interpretación hidrológica de las clases se realizaron Análisis de Correspondencias (AC) entre las clases de la REC-Chile y las condiciones medias del hidrograma de los ríos de Chile. Este análisis define correspondencias entre dos conjuntos de variables, categóricas y/o continuas, representándolas en forma bidimensional, lo cual permite realizar interpretaciones hidrológicas de las clases de la REC-Chile. Los AC se realizaron mediante la librería ca (Greenacre \& Nenadic 2007) ambientada en el lenguaje estadístico R versión 2.8.1 ( $\mathrm{R}$ Development Core Team, 2008).

Se realizaron dos AC de acuerdo con los objetivos de clasificación definidos anteriormente. El primero se realizó entre las clases pertenecientes al esquema CLPR y las variables que describen la magnitud del caudal en el tramo de río (Q50, R50max, y R50min). El segundo AC se realizó entre las clases pertenecientes al esquema CLOF y las variables asociadas a la estacionalidad y variabilidad intranual del hidrograma (R50max, R50min, T50max y T50min). En este tipo de análisis no fue considerado el esquema $\mathrm{CL}$, ya que junto al esquema CLPR ambos permiten una discriminación de las variables que describen la magnitud del caudal en el tramo de río, pero el esquema CLPR permite una interpretación más precisa de las clases.

En ambos AC las variables continuas fueron recodificadas. El proceso consiste en redefinir las escalas como un par de escalas complementarias, el 
CAPÍtUlo 3 EVALUACIÓN DE LA REC-ChILE polo positivo $(+)$ y el polo negativo (-). Esta recodificación permite definir ejes entre los polos de una misma variable, interpretándose como un gradiente en donde el origen de los ejes es la media de las variables (Greenacre 2008).

\subsubsection{Evaluación geoquímica de la REC-Chile}

El parámetro utilizado para evaluar la calidad geoquímica natural de las aguas fue la conductividad eléctrica ( $\mathrm{CE}$, en $\mu \mathrm{S} / \mathrm{cm}$ ). Ésta es una medida de la suma total de iones en una masa hídrica, la cual depende de variables físicas, químicas y biológicas (Vila \& Molina 2006).

Para lograr una adecuada discriminación de la variación espacial de la calidad geoquímica, se consideraron los factores controladores Clima, Origen de Flujo y Geología (Peredo-Parada et al. 2010). Estos tres factores conforman el tercer nivel de clasificación de la REC-Chile que agrupa las 3 escalas espaciales más altas de la clasificación.

\subsubsection{Datos y parámetros fisicoquímicos}

Los datos utilizados corresponden a dos fuentes de información. La primera corresponde a mediciones realizadas en las estaciones de calidad fisicoquímica de las aguas (administradas por la DGA) distribuidas a lo largo de toda la REC-Chile y publicadas en el informe "Diagnóstico y clasificación de los cuerpos de agua según objetivos de calidad" (DGA 2004). La segunda fuente de información corresponde a muestreos puntuales 
Capítulo 3 EVAluación de LA REC-Chile

realizados entre los años 1982 y 2008, los cuales fueron publicados en diversos artículos científicos y/o en informes técnicos. De la base de datos resultante se han eliminado los puntos cercanos a desembocaduras al mar para eliminar posibles efectos de intrusión marina y puntos ubicados aguas abajo de centros urbanos para eliminar el efecto de contaminación antrópica. El número total de datos corresponden a 478, de los cuales 170 son obtenidos de las estaciones de calidad del agua (DGA 2004) y 308 de muestreos puntuales.

\subsubsection{Test de clasificación geoquímica}

El test de clasificación utilizado, al igual que en la clasificación hidrológica, es el inverso al test de similaridad de Van Sickle (1997). Las clases RECChile utilizadas en este test son aquellas correspondientes al tercer nivel de clasificación, es decir, a la superposición jerárquica de los factores Clima, Origen de Flujo y Geología.

La matriz de disimilaridad se obtuvo mediante la distancia Bray-Curtis, eliminando del análisis aquellas clases que solo presentan un dato. Los cálculos de las disimilaridades $\bar{W}_{i}, \bar{W}, \bar{B}$, el análisis de permutaciones aleatorias (bootstrap), así como la determinación de los dendrogramas se realizaron mediante el paquete de funciones Env.Class desarrolladas por Snelder (comunic. Personal) para el programa estadístico R, versión 2.8.1 (R Development Core Team 2008). 
3.3.8. Interpretación geoquímica de las clases REC-Chile

A cada punto de muestreo se le asoció su código REC-Chile correspondiente, a un tercer nivel de clasificación. Para las clases con más de 5 datos se determinaron la CE mínima, máxima, mediana y promedio, además de los percentiles P25 y P75. Dichos datos se mostraron gráficamente, de acuerdo a sus niveles REC-Chile, en diagramas de cajas y bigotes (box-plot) agrupados en sus clases correspondientes. 


\subsection{RESULTADOS}

\subsubsection{Test de Clasificación hidrológica}

En este test se pudieron incluir todas las clases en el esquema CL, es decir, que el $100 \%$ de las clases contaban con datos suficientes. En el esquema CLPR fueron incluidas 12 clases, lo que equivale a una representatividad del 67\% (12/18) del total de las clases representadas en los datos; el resto de las clases presentaron sólo un dato, por lo cual fueron excluidas del test. En el esquema CLOF fueron incluidas sólo 7 clases, lo que equivale a un $41 \%$ (7/17) de representatividad del total de las clases disponibles.

Los resultados del test de clasificación se muestran en la Tabla 3.1. En ella se aprecia que en los tres esquemas en análisis de la REC-Chile (CL, CLPR y CLOF) los valores de $\bar{B}$ exceden a los valores de $\bar{W}$, por lo tanto, los tres presentan estructura de clasificación bajo el grupo de variables definidas para cada uno. De los tres esquemas, CLOF se comporta mejor como clasificación mostrando un valor de $\mathrm{M}$ menor que los otros dos $\left(\mathrm{M}_{\mathrm{CLOF}}<\right.$ $\mathrm{M}_{\text {CLPR }}$ ), y a su vez, CLPR presenta una estructura de clasificación ligeramente mejor que CL $\left(\mathrm{M}_{\mathrm{CLPR}}<\mathrm{M}_{\mathrm{CL}}\right)$.

Al comparar el índice M entre los esquemas de clasificación de la RECChile y sus equivalentes de la clasificación CHID, se aprecia que la capacidad de discriminación de la REC-Chile es mayor en ambos patrones hidrológicos (magnitud y estacionalidad del flujo) mostrando mayores 
CAPÍtUlo 3 EVALUACIÓN DE LA REC-ChILE diferencias en el poder de discriminación de la estacionalidad del flujo. Como se esperaba, las clasificaciones a posteriori (Cluster CLPR y Cluster CLOF) tienen mejor estructura de clasificación con respecto a las clasificaciones a priori (CLPR y CLOF, respectivamente).

\begin{tabular}{|c|c|c|c|c|c|}
\hline $\begin{array}{c}\text { Esquemas de } \\
\text { clasificación }\end{array}$ & $\begin{array}{l}\mathbf{N}^{0} \text { de } \\
\text { clases }\end{array}$ & $\begin{array}{c}\text { Tipo de } \\
\text { clasificación }\end{array}$ & $\bar{B}$ & $\overline{\boldsymbol{W}}$ & $\boldsymbol{M}=\overline{\boldsymbol{W}} / \overline{\boldsymbol{B}}$ \\
\hline CL & 5 & A priori & 0.390 & 0.263 & 0.674 \\
\hline CLPR & 12 & A priori & 0.384 & 0.248 & 0.633 \\
\hline CLOF & 7 & A priori & 0.273 & 0.139 & 0.509 \\
\hline CHID_CLPR & 14 & A priori & 0.360 & 0.304 & 0.845 \\
\hline CHID_CLOF & 14 & A priori & 0.253 & 0.251 & 0.992 \\
\hline Cluster CLPR & 12 & A posteriori & 0.364 & 0.109 & 0.298 \\
\hline Cluster CLOF & 7 & A posteriori & 0.289 & 0.117 & 0.404 \\
\hline
\end{tabular}

Los dendrogramas de cada esquema de clasificación (Figura 3.2) muestran la disimilaridad para cada clase, en los tres esquemas de clasificación. Para el esquema CL (Fig. 3.2.a), se observa que todas las clases tienen una disimilaridad $\left(\bar{W}_{i}\right)$ menor que la disimilaridad media inter-clases $(\bar{B})$. Las clases con menor variabilidad (es decir, aquellas que presentan una mayor diferencia $\bar{B}-\bar{W}_{i}$ reflejándose en una mayor longitud hacia la izquierda de la rama en el dendrograma) están asociadas a climas más secos. La variabilidad tiende a aumentar hacia climas más húmedos. En el esquema CLPR (Figura 3.2b), las clases con una mayor variabilidad son $\mathrm{Ar} / \mathrm{Hw}$, $\mathrm{Mw} / \mathrm{Hw}$ y $\mathrm{Vw} / \mathrm{Lr}$; esta última tiene una variabilidad mayor que la media inter-clases $\left(\bar{W}_{i}>\bar{B}\right)$. En climas húmedos (W y Vw) la variabilidad aumenta hacia aguas abajo de la cuenca $(\mathrm{Ww} / \mathrm{Hw}, \mathrm{Vw} / \mathrm{Ur}, \mathrm{Vw} / \mathrm{Mr}, \mathrm{Vw} / \mathrm{Lr}$, 
CAPÍtUlo 3 EVAluación de LA REC-ChILE

en orden ascendente de variabilidad), sin embargo, en climas áridos (Ar, Sa y $\mathrm{Mw}$ ) la tendencia parece invertirse, estando las clases más variables en las zonas altas de la cuenca y hacia las zonas bajas se vuelven más homogéneas ( $\mathrm{Ar} / \mathrm{Mr}, \mathrm{Ar} / \mathrm{Ur}$ y $\mathrm{Ar} / \mathrm{Hw}$, en orden ascendente).
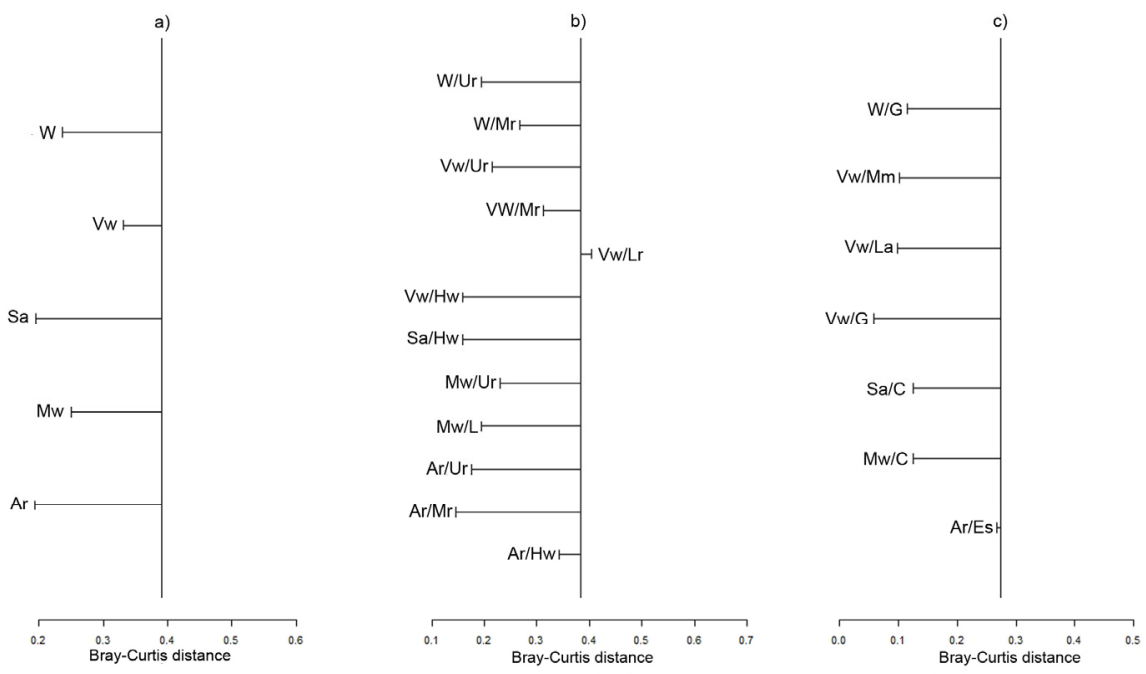

Figura 3.2. Dendrogramas para los esquemas CL (a), CLPR (b) y CLOF (c). Interpretación de las clases para cada esquema de clasificación, ver capitulo 2

En el esquema de clasificación CLOF (Figura 3.2c), la clase Ar/Es presenta una disimilaridad media alta, casi igual a $\bar{B}$, por tanto, presenta una alta variabilidad. El resto de las clases presentan valores bajos de disimilaridad $\left(\bar{W}_{i}\right)$ resultando clases con una homogeneidad interna alta. En este esquema, no se observa una tendencia de aumento o disminución de la variabilidad según el clima u origen del flujo. 
3.4.2. Interpretación hidrológica de las clases

Para este tipo de análisis se han utilizado todas las clases que aparecen asociadas a los tramos de las estaciones de aforo; existen clases con un solo dato y otras con dos o más, por tanto, los resultados de estos análisis de correspondencia $(\mathrm{AC})$ se analizarán teniendo en cuenta esta condición de las clases.

Los resultados del primer AC, realizado entre las clases del esquema CLPR y las variables asociadas a la magnitud del hidrograma (Q50, R50min y R50max), indican que se puede reducir a un problema de dos dimensiones describiendo aproximadamente un 70\% de la varianza (69.43\%).

La representación gráfica de este análisis (gráfico en columnas principales) se muestra en la Figura 3.3. En ella se distinguen 4 agrupaciones delimitadas por elipses. El primer grupo de clases está definido entre los ejes -R50max, +R50min y -Q50, definiendo clases con caudales medios anuales bajos y variaciones estacionales pequeñas en torno al caudal medio. Las categorías en este cuadrante son: $\mathrm{Ar} / \mathrm{Hw}, \mathrm{Ar} / \mathrm{Ur}, \mathrm{Ar} / \mathrm{Mr}, \mathrm{Sa} / \mathrm{Hw}$ (códigos en Tabla 2.1 del capitulo 2). Las clases $\mathrm{Ar} / \mathrm{Lr}$ y Sa/Mr también están en este grupo, sin embargo, como solo existe un dato para esta categoría, solo es posible mencionar que podría existir una tendencia a pertenecer a este grupo de clases. Un segundo grupo, delimitado entre los ejes -Q50 y +R50max, define clases con caudal medio anual bajo y un aumento en el caudal medio mensual máximo. Las clases presentes en este grupo son $\mathrm{Mw} / \mathrm{Ur}$ y Mw/Lr. Por el reducido tamaño muestral, $\mathrm{Mw} / \mathrm{Hw}, \mathrm{Mw} / \mathrm{Mr}$ y W/Hw sólo presentan tendencia a estar en este grupo. Un tercer grupo definido entre los ejes 
CAPITULO 3 EVALUACIÓN DE LA REC-CHILE

+ R50max y -R50min define clases con mayor caudal medio anual que los grupos anteriores y variaciones estacionales extremas. Las 3 clases aquí presentes son aquellas consideradas como zonas altas de las cuencas con climas húmedos (W/Mr, W/Ur y Vw/Hw).

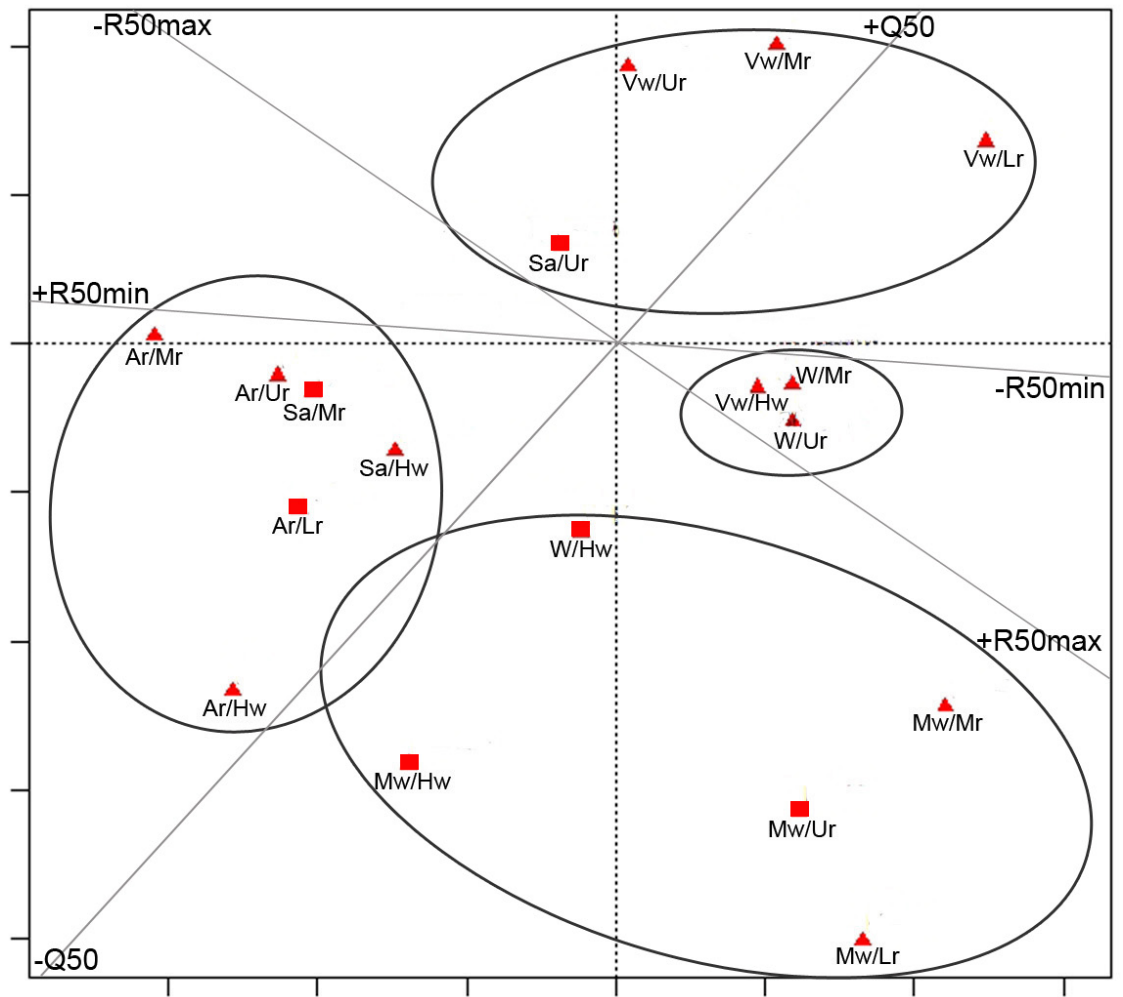

Figura 3.3. Análisis de Correspondencia entre las variables asociadas a magnitud del hidrograma (Q50, R50min y R50max) y las clases resultantes de la combinación de los factores Clima y Posición Relativa de subsubcuenca (CLPR) de la RECChile. Los ejes gradientes definidos en la figura como $(+250,-250) ; \quad(+R 50 \max ;-R 50 \max )$ y (+R50min, -R50min) son obtenidos a partir del redoblado de las variables Q50, R50max y R50min respectivamente. Las clases mostradas con un cuadrado solo presentan un dato, aquellas mostradas con un triángulo presentan dos o más datos. 
CAPÍtulo 3 EVALUACIÓN dE LA REC-ChILE El cuarto y último grupo está delimitado por los ejes -R50min, -R50max y + Q50, definiendo clases con el mayor caudal medio, variaciones estacionales moderadas y con bajo caudal base. A este grupo pertenecen las clases de zonas altas, medias y bajas de climas muy húmedos ( $\mathrm{Vw} / \mathrm{Ur}$, $\mathrm{Vw} / \mathrm{Mr}$ y $\mathrm{Vw} / \mathrm{Lr}$ ).

Tratando de generalizar los resultados, en el primer AC se puede decir que hay un aumento del caudal medio anual (Q50) desde la categoría Árido (Ar) hasta la categoría Muy Húmedo (Vw), así como de sus variaciones estacionales. La totalidad de las clases que contienen la categoría Árido presentan caudales bajos y variaciones estacionales pequeñas. En las clases con la categoría Semiárido $(\mathrm{Sa})$ no es posible realizar un análisis estadístico robusto debido a la escasa cantidad de estaciones de aforo que tiene (4/48). Las clases con la categoría Subhúmedo $(\mathrm{Mw})$ siguen presentando caudales bajos, aunque mayores que la categoría Ar, con caudales medios mensuales máximos que van en aumento. Las clases con las categorías Húmedo (W) y Muy húmedo $(\mathrm{Vw})$ muestran una mayor variación estacional en las zonas altas de la cuenca, la cual va disminuyendo hacia aguas abajo.

Los resultados del segundo AC, entre las clases del esquema CLOF y las variables asociadas a las estacionalidad del hidrograma (R50min, R50max, T50min y T50max), se han representado en un gráfico bidimensional (Fig. 3.4) manteniendo una varianza igual a $54.2 \%$.

En este caso no se observan agrupaciones de clases tan claras; sin embargo, se distinguen 4 cuadrantes definidos por los ejes gradientes de las variables explicatorias (R50min y R50max). El primer cuadrante, delimitado entre los 
ejes -R50min y -R50max, define clases con caudal base bajo y caudal medio mensual máximo atenuado. En este cuadrante se encuentran las clases $\mathrm{Vw} / \mathrm{La}, \mathrm{Vw} / \mathrm{Mm}, \mathrm{Sa} / \mathrm{M}$. Las clases $\mathrm{Sa} / \mathrm{Mm}$ y $\mathrm{Ar} / \mathrm{L}$ también se encuentra dentro de este cuadrante, pero como existe solo un dato para estas clases, solo es posible mencionar que podría mostrar una tendencia a pertenecer a este cuadrante. El segundo cuadrante está delimitado por los ejes $+\mathrm{R} 50 \mathrm{~min}$ y -R50max. Éste define clases con variaciones estacionales pequeñas, es decir, con caudales medios mensuales mínimos y máximos cercanos al caudal medio anual, encontrándose la clase Ar/Es. En dicho cuadrante solo puede decirse que Ar/M tiene tendencia a pertenecer a este grupo.

El tercer cuadrante, delimitado por + R50min y + R50max define clases con caudal base mayor y caudales medios mensuales máximos elevados; las clases presentes en este cuadrante son $\mathrm{Mw} / \mathrm{M}, \mathrm{W} / \mathrm{G}$ y $\mathrm{Vw} / \mathrm{G}$. El cuarto y último cuadrante delimitado por -R50min y + R50max, define clases con caudales mínimos bajos y caudales máximos altos, y por lo tanto con tendencia a tener variaciones estacionales extremas $(\mathrm{Vw} / \mathrm{V}, \mathrm{Mw} / \mathrm{L}, \mathrm{W} / \mathrm{L}$, $\mathrm{W} / \mathrm{V}, \mathrm{W} / \mathrm{M}, \mathrm{Vw} / \mathrm{Mm})$; se trata en general de clases con categorías procedentes de lluvias. 
CAPÍTULO 3 EVALUACIÓN DE LA REC-CHILE

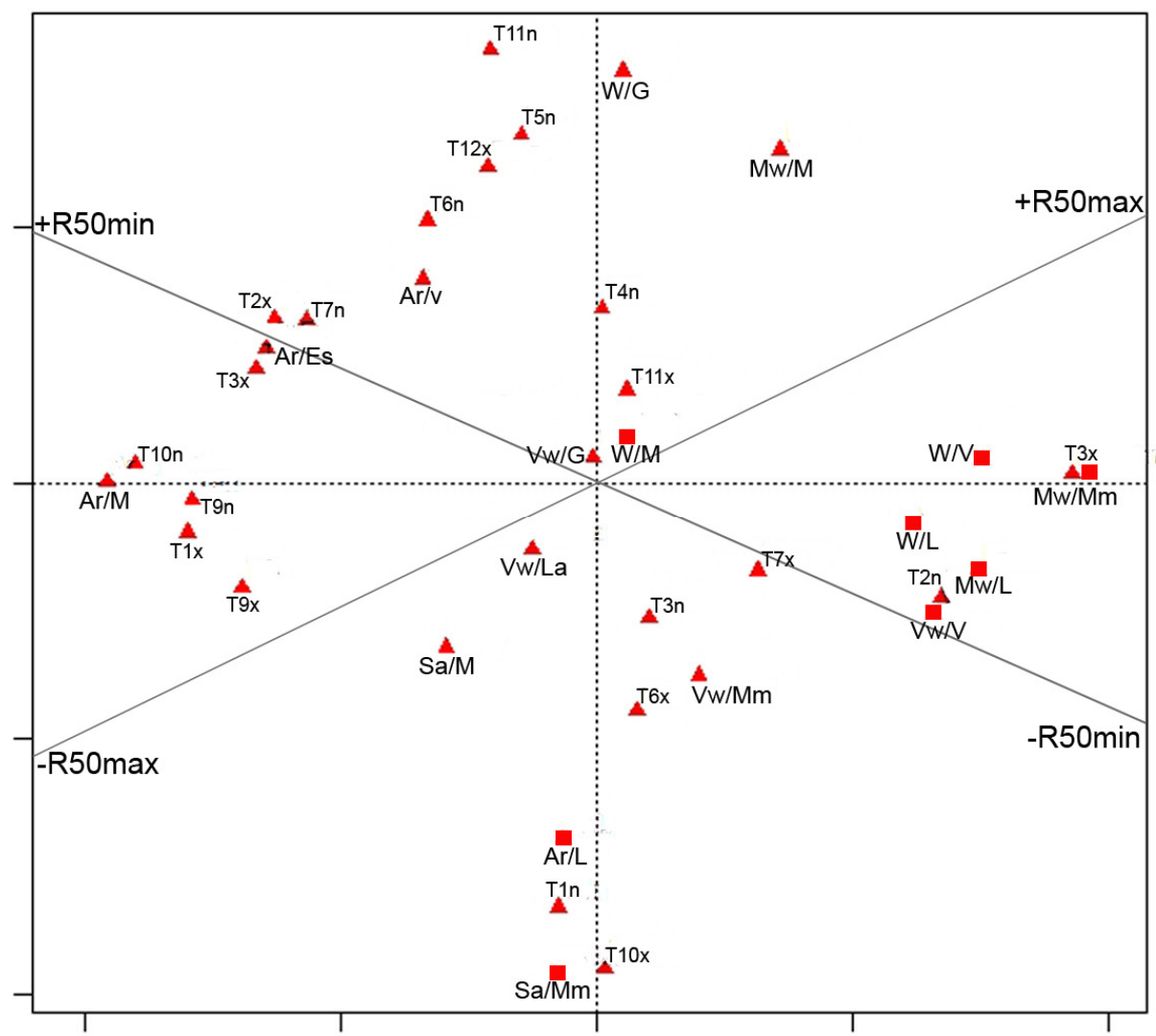

Figura 3.4. Análisis de Correspondencia entre las variables asociadas a la estacionalidad del hidrograma (R50min, R50max, T50min y T50max) y las clases resultantes de la combinación de los factores Clima y Origen de flujo (CLOF) de la REC-Chile. Meses de ocurrencia de caudales medios mensuales máximos discretizados en año natural (Enero=T1x, Febrero=T2x.... Diciembre=T12x). Meses de ocurrencia de caudales medios mensuales mínimos discretizados en año natural (Enero=T1n, Febrero=T2n...Diciembre=T12n). Los ejes gradientes definidos en la figura como (+R50max;-R50max) y (+R50min, -R50min) son obtenidos a partir del redoblado de las variables R50max y R50min respectivamente. Las clases mostradas con un cuadrado solo presentan un dato, aquellas mostradas con un triángulo presentan dos o más datos.

Este mismo $\mathrm{AC}$ ha permitido determinar las correspondencias existentes entre la estacionalidad del flujo y las clases (Tabla 3.2) mediante su proximidad a la discretización mensual de las variables T50min y T50max. 
Capítulo 3 Evaluación de LA REC-ChILE

Se observa que las clases pertenecientes a climas áridos y provenientes de derretimiento de nieves, tienen caudales medios mensuales mínimos que ocurren en invierno, y los máximos en verano. Para clases con este mismo origen de flujo, pero de climas más húmedos, los meses de caudales máximos se adelantan al comienzo del verano o fines de primavera. Las clases con flujo pluvial (L, V, Mm), tienen sus caudales medios mensuales mínimos en verano y los máximos en invierno. La categoría Glaciar $(\mathrm{G})$ tiene caudales mínimos en otoño y máximos en primavera y comienzo del verano.

\begin{tabular}{|c|c|c|c|c|}
\hline Clase & Cuadrante & Variaciones estacionales & Tmin & Tmax \\
\hline $\mathrm{Vw} / \mathrm{La}$ & I & $\begin{array}{l}\text { Moderadas. Altos caudales } \\
\text { medios mensuales mínimos y } \\
\text { máximos }\end{array}$ & Marzo & $\begin{array}{l}\text { Julio a } \\
\text { septiembre }\end{array}$ \\
\hline $\mathrm{Vw} / \mathrm{Mm}$ & I & $\begin{array}{l}\text { Moderadas. Altos caudales } \\
\text { medios mensuales mínimos y } \\
\text { máximos }\end{array}$ & Marzo & Junio, julio \\
\hline $\mathrm{Sa} / \mathrm{M}$ & I & $\begin{array}{l}\text { Moderadas. Altos caudales } \\
\text { medios mensuales mínimos y } \\
\text { máximos }\end{array}$ & S/definir & Septiembre \\
\hline $\begin{array}{l}\mathrm{Sa} / \mathrm{Mm}^{*}, \\
\mathrm{Ar} / \mathrm{L}^{*}\end{array}$ & I & $\begin{array}{l}\text { Pequeñas. Altos caudales medios } \\
\text { mensuales mínimos y bajos } \\
\text { caudales medios mensuales } \\
\text { máximos }\end{array}$ & Noviembre & Octubre \\
\hline $\mathrm{Ar} / \mathrm{Es}$ & II & $\begin{array}{l}\text { Pequeñas. Altos caudales medios } \\
\text { mensuales mínimos y bajos } \\
\text { caudales medios mensuales } \\
\text { máximos }\end{array}$ & Julio & $\begin{array}{l}\text { Febrero, } \\
\text { marzo }\end{array}$ \\
\hline $\mathrm{Ar} / \mathrm{M}^{*}$ & II & $\begin{array}{l}\text { Pequeñas. Altos caudales medios } \\
\text { mensuales mínimos y bajos } \\
\text { caudales medios mensuales } \\
\text { máximos }\end{array}$ & $\begin{array}{l}\text { Sept., } \\
\text { octubre }\end{array}$ & Enero \\
\hline $\mathrm{Mw} / \mathrm{M}$ & III & $\begin{array}{l}\text { Moderadas. Bajos caudales } \\
\text { medios mensuales máximos y } \\
\text { mínimos }\end{array}$ & Abril & Noviembre \\
\hline
\end{tabular}


CAPítulo 3 EVALUACIÓN DE LA REC-CHILE

Tabla 3.2. Estacionalidad de las clases REC-Chile a un segundo nivel de clasificación (CLOF) según cuadrante, y según correspondencia con los meses de ocurrencia de caudales medios mensuales mínimos (Tmin) y máximos (Tmax), realizado mediante un análisis de correspondencias

\begin{tabular}{|c|c|c|c|c|}
\hline Clase & Cuadrante & Variaciones estacionales & Tmin & Tmax \\
\hline $\mathrm{W} / \mathrm{G}$ & III & $\begin{array}{l}\text { Moderadas. Bajos caudales } \\
\text { medios mensuales máximos y } \\
\text { mínimos }\end{array}$ & Mayo & Diciembre \\
\hline $\begin{array}{l}\mathrm{Vw} / \mathrm{G}, \\
\mathrm{W} / \mathrm{M}^{*}\end{array}$ & III & $\begin{array}{l}\text { Moderadas. Bajos caudales } \\
\text { medios mensuales máximos y } \\
\text { mínimos }\end{array}$ & $\begin{array}{l}\text { Marzo, } \\
\text { Abril }\end{array}$ & Noviembre \\
\hline $\begin{array}{l}\mathrm{Vw} / \mathrm{V}^{*}, \\
\mathrm{Mw} / \mathrm{L}^{*}, \\
\mathrm{~W} / \mathrm{L}^{*}, \\
\mathrm{~W} / \mathrm{V}^{*}, \\
\mathrm{Mw} / \mathrm{Mm}^{*}\end{array}$ & IV & $\begin{array}{l}\text { Extremas. Altos caudales medios } \\
\text { mensuales máximos y bajos } \\
\text { caudales medios mensuales } \\
\text { mínimos }\end{array}$ & $\begin{array}{l}\text { Febrero, } \\
\text { marzo }\end{array}$ & $\begin{array}{l}\text { Julio, } \\
\text { agosto }\end{array}$ \\
\hline
\end{tabular}

* Clases con solo un dato.

Es posible englobar algunos resultados generales de este AC. Las clases que contienen categorías de flujo pluvial (L, V, Mm) presentan caudales medios mensuales máximos entre junio y agosto y mínimos entre febrero y abril, correspondiendo a los meses de ocurrencia de lluvias (invierno) y estiaje (verano), respectivamente.

La categoría Cordillera $(\mathrm{M})$ presenta caudales medios mensuales máximos en octubre y noviembre, correspondientes a los derretimientos de la nieve acumulada y caudales mínimos en los meses febrero, marzo y abril, coincidentes con los meses de estiaje. Las clases $\mathrm{Ar} / \mathrm{M}$ y $\mathrm{Ar} / \mathrm{Es}$ presentan diferencias en los meses de ocurrencia de los caudales medios mensuales máximos y mínimos. En ambas clases, los máximos se producen entre enero y marzo y los mínimos entre septiembre y noviembre. Este patrón de precipitación estival es conocido informalmente como 'Invierno Altiplánico' o 'Invierno Boliviano' y representa la humedad proveniente del Amazonas 
Capítulo 3 EVAluación de LA REC-Chile

(Ronchail 1989). La categoría Lagos (La) se comportan de forma similar que las categorías de flujo pluvial, pero con un desfase de un mes en la ocurrencia de los caudales medios extremos (efecto amortiguador y retardador del lago). Por último, en la categoría Glaciar (G) los caudales máximos ocurren en diciembre (finales de primavera) y los mínimos entre junio y julio.

\subsubsection{Test de clasificación geoquímica}

El test de clasificación muestra que los tres niveles de clasificación logran una buena discriminación espacial de la CE (Tabla 3.3). A medida que aumenta el nivel de clasificación mejora el índice $\mathrm{M}$; cabe recordar que dichos niveles, de mayor a menor escala, incluyen los factores Clima, Origen de Flujo y Geología.

\begin{tabular}{lcccc} 
Tabla 3.3 & Resultados del test de clasificación \\
basado en disimilaridades para los tres primeros \\
niveles de la clasificación REC-Chile, donde $\bar{B}$ es \\
la disimilaridad media inter-clases, $\bar{W}$ es la \\
$\begin{array}{l}\text { disimilaridad media intra-clases, M es la razón } \\
\text { entre estas dos disimilaridades. }\end{array}$ \\
\hline $\begin{array}{c}\text { Nivel } \\
\text { REC-Chile }\end{array}$ \\
de clases & $\bar{B}$ & $\bar{W}$ & M \\
\hline $\begin{array}{r}\text { Primero } \\
\text { Segundo }\end{array}$ & 5 & 0.562 & 0.364 & 0.648 \\
Tercero & 19 & 0.528 & 0.252 & 0.478 \\
\hline
\end{tabular}


Capítulo 3 Evaluación de La REC-Chile 3.4.4. Interpretación geoquímica de las clases

En el primer nivel de clasificación se aprecia que los valores medios de CE disminuyen a medida que las categorías se relacionan con climas más húmedos (Figura 3.5) con un notorio aumento en la categoría Árido

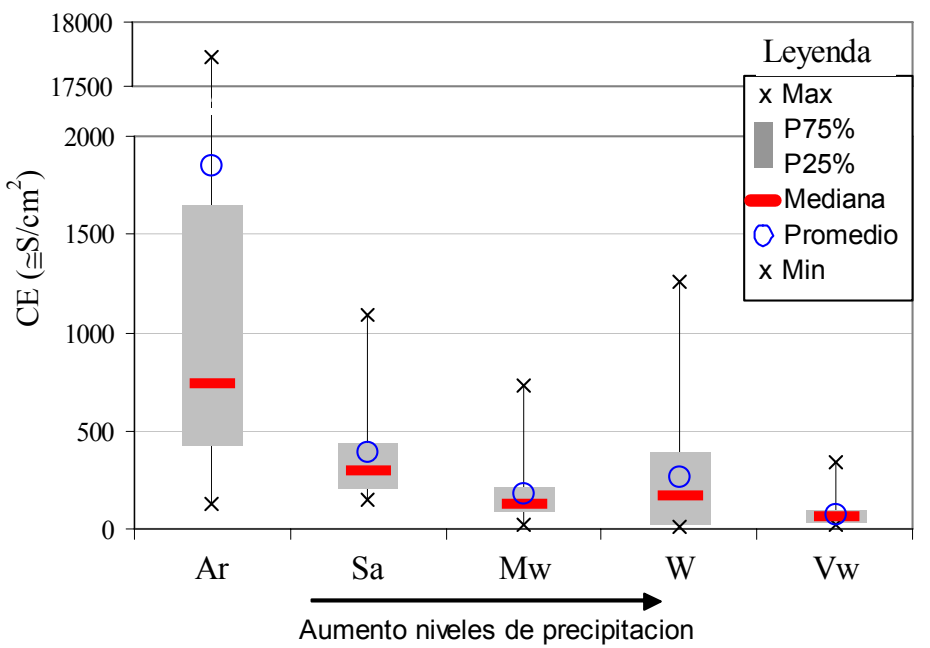

Figura 3.5. Gráfico de cajas y bigotes de la Conductividad Eléctrica en un primer nivel de clasificación REC-Chile. Ar=Árido; Sa=Semiárido; $\mathrm{Mw}=$ Subhúmedo; $\mathrm{W}=$ Húmedo; $\mathrm{Vw}=\mathrm{Muy}$ Húmedo

Al pasar al segundo nivel de clasificación (Figura 3.6) se aprecia que el origen del flujo también juega un papel importante. En climas húmedos (W, $\mathrm{Vw}$ ) los valores de CE son bajos en las clases donde el flujo es proveniente de precipitaciones $(\mathrm{W} / \mathrm{L}, \mathrm{W} / \mathrm{V}, \mathrm{W} / \mathrm{Mm}, \mathrm{Vw} / \mathrm{V}$ y $\mathrm{Vw} / \mathrm{Mm})$. A medida que aumenta el nivel de precipitación disminuye el valor medio de CE. Para clases provenientes del derretimiento de nieves (W/M, W/G, Vw/G) el valor de la $\mathrm{CE}$ tiende a aumentar. En categorías asociadas a climas secos (Ar y 
CAPÍtUlo 3 EVAlUación de LA REC-Chile

$\mathrm{Sa}$ ) hay una tendencia similar de acuerdo a la proveniencia del flujo (Figura 3.6a), sin embargo los valores medios de CE presentan valores similares tanto en clases asociadas a precipitación (Ar/L) como aquellas asociadas al derretimiento de nieves (Ar/Es). En la categoría de clima subhúmedo (Mw) (Figura 3.6b) existe una zona de transición en donde el comportamiento de los valores de CE presenta un comportamiento errático

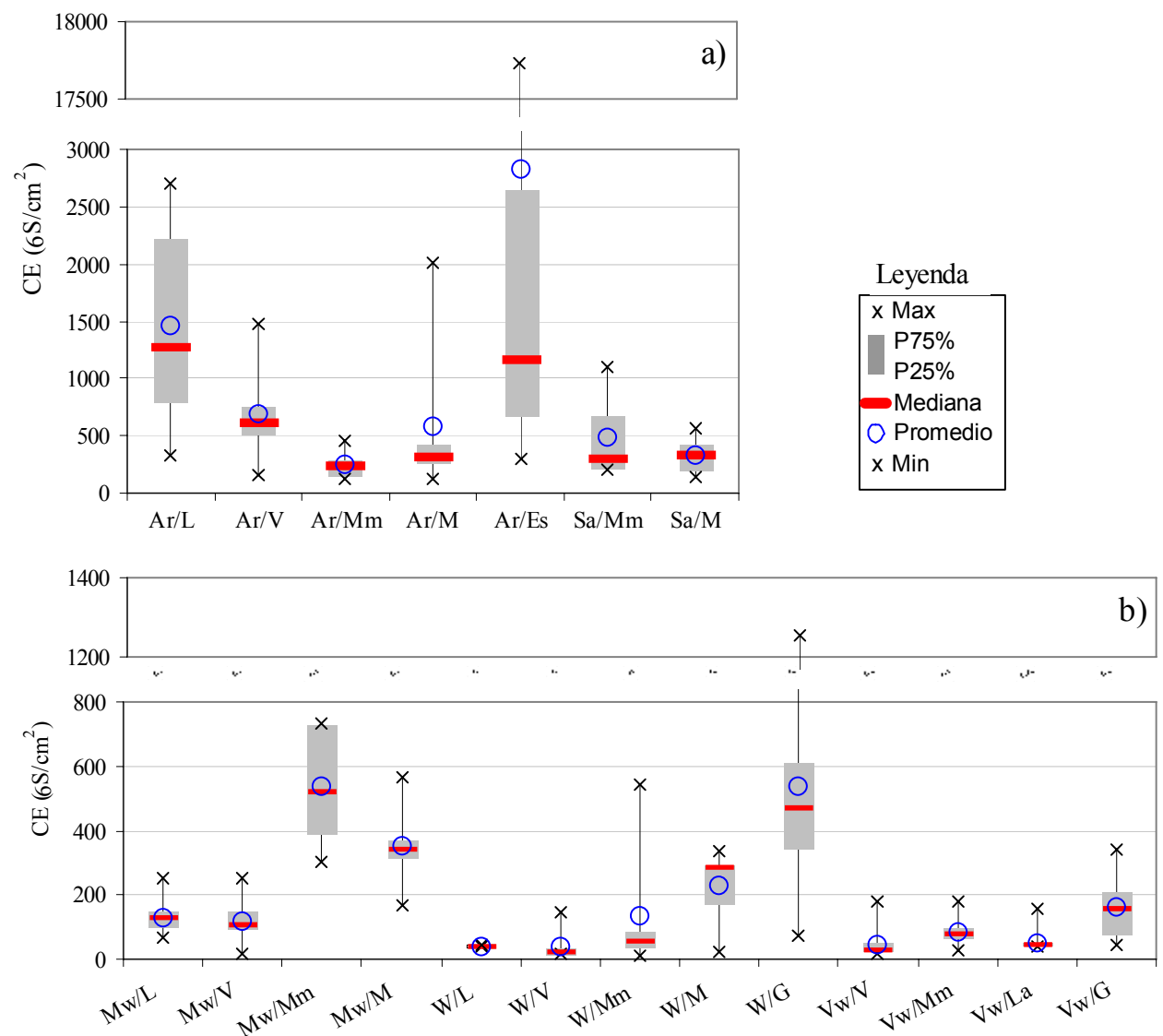

Figura 3.6. Gráfico de cajas y bigotes de la Conductividad eléctrica (CE) clasificadas mediante el segundo nivel de la REC-Chile. Clases asociadas a categorías de climas áridos (Fig. a) y clases asociadas a climas húmedos (Fig. b) . Para interpretación y características de las categorías consultar Tabla 2.1 
Analizando el comportamiento de la CE dentro de una misma clase del factor Clima, se aprecia que existen dos tendencias. La primera está relacionada a las clases asociadas a los rangos de precipitación acumulada ( $\mathrm{L}, \mathrm{V}, \mathrm{Mm})$, en donde a medida que ésta aumenta disminuye el valor medio de $\mathrm{CE}$, lo que es concordante con los niveles de precipitación que van lavando el terreno. La segunda tendencia está relacionada con las clases asociadas al deshielo proveniente de la cordillera de los Andes (M, Es, G); se observa que la CE aumenta a medida que aumenta la altitud del deshielo, lo que concuerda con aumentar el tiempo de lixiviación en litologías volcánicas.

Al aumentar al tercer nivel de clasificación (Figura 3.7), solo se analizó una clase del clima Árido (Ar/Es), 2 clases de clima subhúmedo (Mw/L y $\mathrm{Mw} / \mathrm{V})$ y 4 clases de clima muy húmedo (Vw/V, VwMm, Vw/La y Vw/G), ya que éstas tenían al menos dos categorías del factor Geología con más de 5 datos por clase, número mínimo para este análisis.

En la clase Ar/Es (Figura 3.7a) se observa que las rocas carbonatadas (Ca) tienen un alto valor promedio de CE, con máximos cercanos a 18000 $\mu \mathrm{S} / \mathrm{cm}^{2}$. La CE va disminuyendo para las clases volcánicas fracturadas (Vf), rocas mixtas sedimentarias-volcánicas $(\mathrm{Mx})$, rocas sedimentarias $(\mathrm{Ms})$ y finalmente Plutónicas (Pl). 
Capítulo 3 Evaluación de LA REC-ChILE

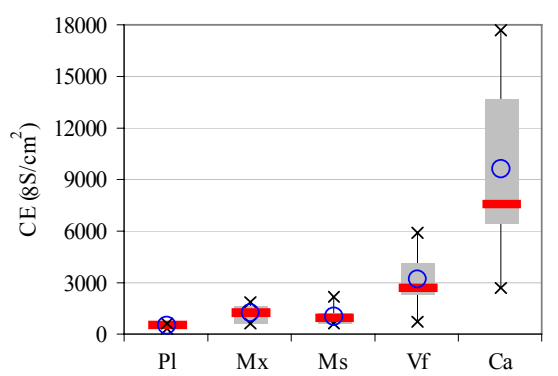

a)

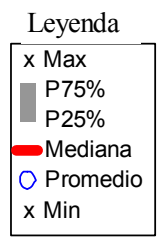

b)

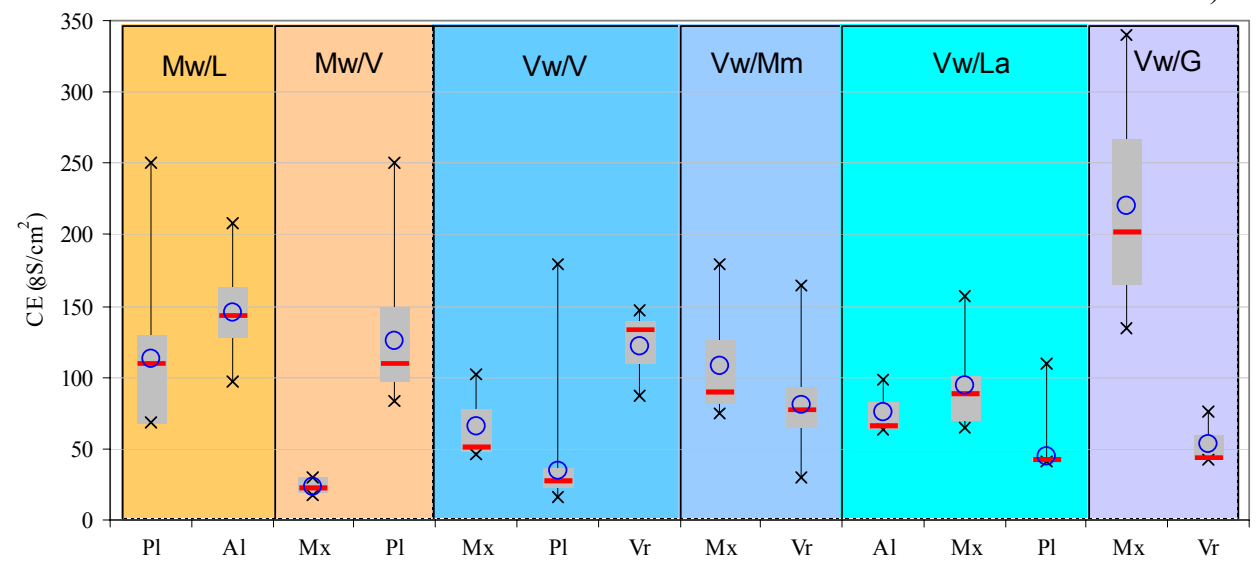

Figura 3.7. Gráfico de cajas y bigotes para los valores de conductividad eléctrica agrupados en las categorías del factor Geología. Para interpretación y características de las categorías, consultar Tabla 2.1.

En la clase subhúmeda (Mw) (Figura 3.7b), solo existen clases provenientes de la precipitación (Mw/L y Mw/V). En ellas, el comportamiento de la $\mathrm{CE}$ viene dado por valores promedios similares para las rocas plutónicas (Pl), un poco más elevado para las terrenos aluviales (Al) y valores bajos para las rocas mixtas Sedimento-volcánicas $(\mathrm{Mx})$. En las clases muy húmedas $(\mathrm{Vw})$ el comportamiento en clases provenientes de la precipitación $(\mathrm{Vw} / \mathrm{V})$ es similar, con bajos valores promedios de CE, sin embargo, los promedios más bajos corresponden a las rocas Plutónicas $(\mathrm{Pl})$, un poco mayores para rocas mixtas Sedimento-volcánicas $(\mathrm{Mx})$ y los valores máximos para rocas volcánicas (Vr). En clases provenientes del deshielo (Vw/M y Vw/G), los 102 
CAPÍtUlo 3 EVALUACIÓN DE LA REC-ChILE promedios mayores ocurren en rocas mixtas $(\mathrm{Mx})$, y los promedios más bajos ocurren en rocas volcánicas $(\mathrm{Vr})$. La clase $\mathrm{Vw} / \mathrm{La}$, se comporta de forma similar que las clases provenientes de precipitación, es decir con valores promedios bajos para rocas Plutónicas (P1), aumentando en terrenos Aluviales (Al) y mayores para rocas mixtas (Mx).

En general se puede comentar que las rocas plutónicas, volcánicas y terrenos aluviales presentan los promedios más bajos para una misma clase, en el segundo nivel de clasificación; mientras tanto las rocas mixtas, volcánicas fracturadas y carbonatadas presentan los valores más altos dentro de una misma clase, en el segundo nivel de clasificación. 


\subsection{DISCUSIÓN}

Distintos niveles jerárquicos de clasificación de la REC-Chile permiten discriminar estadísticamente significativa distintos objetivos de clasificación. La elección de los factores controladores para describir los distintos procesos fluviales se basó en la suposición de que el clima (mediante el factor Clima), la topografía (factor Origen de Flujo) y la Geología son las causas dominantes de los procesos hidrológicos a macro y mesoescala (Peredo-Parada et al. 2010, Snelder \& Biggs 2002). Además se agregó el factor controlador Posición Relativa de Subsubcuenca (PRScuenca) para lograr un mejor ajuste en los caudales medios a lo largo de una cuenca y en el efecto amortiguador en el cauce que se produce hacia aguas abajo.

El esquema CL, equivalente al primer nivel de clasificación, tanto como el esquema CLPR, construidos a partir de la combinación jerárquica de los factores Clima y PRScuenca, logran resultados satisfactorios en la discriminación de la magnitud media de los caudales. El esquema CLOF, equivalente al segundo nivel de clasificación, pareciera discriminar satisfactoriamente la estacionalidad del flujo. Por último, el tercer nivel de clasificación REC-Chile (compuesto por los factores Clima, Origen de Flujo y Geología) logra buenos resultados al momento de discriminar la calidad geoquímica natural de las aguas.

Como se aprecia, el esquema CLPR no sigue la estructura de los otros tres esquemas, los cuales equivalen a un nivel de clasificación REC-Chile. Si se 
Capítulo 3 EValuación de LA REC-Chile hubiese considerado el $4^{\circ}$ nivel como esquema de clasificación (desarrollado a partir del tercer nivel agregando el factor PRScuenca) en sustitución del esquema CLPR, seguramente se habrían obtenido valores de M menores, con su consiguiente aumento en el grado de clasificación. Sin embargo, la elección de los factores Clima y PRScuenca para la conformación del esquema que discrimina magnitudes de caudales obedece principalmente a la poca cantidad de datos disponibles para su evaluación. Esta razón obligó a disminuir el número de clases del esquema a utilizar. Por ello se consideraron solo los factores Clima y PRScuenca pues son los factores que más influyen sobre la discriminación del caudal en un tramo de río (Peredo-Parada et al. 2010). El esquema CLPR tiene un total de 40 clases, de la cuales 17 se están representadas en los datos utilizados en la evaluación. Si se hubiesen agregado jerárquicamente los factores Origen de Flujo y Geología (conformando el cuarto nivel de clasificación) el número de total de clases asciende a 1280, de los cuales 39 estarían representados en los datos. Con esta cantidad de clases, la evaluación resultante no tendría sentido estadístico.

Los resultados del test de clasificación entre los 4 esquemas de clasificación no son comparables entre sí, ya que no fueron realizados con la misma cantidad de datos ni persiguen el mismo objetivo de clasificación. Dentro de los resultados para la discriminación del patrón hidrológico que se muestra en la Tabla 3.1 se observa que el esquema CLOF logra mejores resultados que los otros dos esquemas, lo cual indicaría que la REC-Chile logra resultados con mayor poder de discriminación para la estacionalidad del flujo que para la magnitud. Sin embargo, la cantidad de clases efectivamente evaluadas es poca, solo un $41 \%$ de las clases representadas en los datos. 
Los esquemas CL y CLPR tienen el mismo objetivo de clasificación. De la Tabla 3.1 se desprende que ambos logran resultados satisfactorios en la discriminación de la magnitud de los caudales medios. CLPR logra una mejor discriminación que el esquema CL, pues al aumentar el número de clases se aumenta el grano o precisión en la discriminación (Bryan 2006).

La capacidad, por parte de la REC-Chile, para discriminar los patrones de la calidad geoquímica natural de las aguas fue evaluada considerando otro paquete de datos. Los resultados de la evaluación son similares que para los esquemas CL y CLPR. A medida que se aumenta el número de clases aumenta el poder de discriminación de la REC-Chile (Tabla 3.3), obteniendo mejores resultados con el tercer nivel de clasificación RECChile, lo que concuerda con lo expresado por Peredo-Parada et al. (2010) referente a que la superposición jerárquica de los factores controladores Clima, Origen de Flujo y Geología serían necesarios para lograr una adecuada discriminación de la calidad geoquímica de las aguas en Chile.

El índice $\mathrm{M}$ solo entrega resultados del comportamiento general del esquema como estructura de clasificación, pero no entrega información relevante respecto a cada una de las clases. Una representación gráfica de la variabilidad interna de cada una de las clases se presenta en los dendrogramas (Figura 3.2). Es importante tener en cuenta esta variabilidad de las clases al momento de realizar interpretaciones de éstas. Las cuales se discutirán más adelante. 
CAPÍtUlo 3 EVALUACIÓN DE LA REC-ChILE Los esquemas de clasificación REC-Chile bajo objetivos de clasificación del patrón hidrológico (CL, CLPR y CLOF) presentan mejor estructura de clasificación que sus correspondientes esquemas de la clasificación CHID. Estas diferencias podrían explicarse por la naturaleza de cada clasificación.

La REC-Chile es una clasificación a priori que representa la estructura jerárquica de organización de los ríos (Frissell et al. 1986) mediante la superposición jerárquica de los factores controladores. Además es capaz de reconocer los cambios longitudinales que se producen hacia aguas abajo en un río producto del cambio de las propiedades de las subcuencas (PeredoParada et al. 2010, Snelder et al. 2005, Snelder \& Biggs 2002). En cambio, la clasificación CHID se comporta como una clasificación similar a la definición de una ecorregión, las cuales que son incapaces de representar estos gradientes longitudinales en el río, siendo efectivas sólo cuando los ríos están plenamente dentro de sus límites (ríos de orden 1 a 3), en ríos mayores la ecorregión no es capaz de lograr una correcta discriminación de los procesos fluviales (Snelder \& Biggs 2002).

En Chile no existe una clasificación de ríos y/o cuencas basada en la calidad geoquímica de las aguas, razón por la cual no se realizó una comparación con la REC-Chile bajo este esquema de clasificación. Tampoco se creyó conveniente utilizar la CHID para este objetivo, ya que ésta está desarrollada para clasificar las cuencas desde un objetivo hidrodinámico y no bajo calidad geoquímica, lo cual daría ventajas a la REC-Chile.

La cantidad de clases evaluadas es en general baja con respecto al total posible en cada esquema, limitado por la cantidad de datos disponibles para 
Capítulo 3 EValuación de LA REC-ChILE

la evaluación. Si bien, esta situación no influye mayormente en la comparación de la REC-Chile con la CHID, sí puede ser un factor de importancia en los resultados de la evaluación de la REC-Chile.

Los análisis de correspondencia realizados para los objetivos de clasificación sobre el patrón hidrológico (Figura 3.3 y Figura 3.4) permitieron realizar una interpretación hidrológica de las clases. Los resultados de este análisis están acorde a lo esperado para cada categoría de los distintos factores controladores, tanto para la magnitud del flujo como para estacionalidad. Debido a la poca cantidad de datos disponibles no es posible ser categóricos al momento de asignar las correspondencias entre las variables hidrológicas y las clases REC-Chile, sin embargo, sí se aprecian tendencias de comportamiento definido para agrupaciones de clases, sobretodo en la magnitud del caudal medio y la estacionalidad.

Las categorías del factor Clima presentaron un aumento en el caudal a medida que la precipitación asociada a cada categoría aumenta, de igual forma para las clases del factor PRScuenca en donde el caudal circulante por el tramo de río aumenta a medida que las categorías están asociadas a zonas más bajas de la cuenca, así como la variación estacional disminuye por efecto amortiguador del cauce. Por otra parte, las categorías del factor Origen de Flujo presentaron correspondencias con las variables de estacionalidad de acuerdo a la proveniencia del flujo, la que se detalla en la Tabla 3.2.

Con respecto a la calidad geoquímica de las aguas, el efecto combinado de los dos primeros factores permite interpretar por qué clases de distintos 
Capítulo 3 EVAluación de LA REC-Chile orígenes de flujo y/o litología de roca puedan tener similares valores promedios de CE. Por ejemplo, al analizar la clase Árido (Figura 3.6a), si bien se observa una disminución de la $\mathrm{CE}$ en las clases asociadas a niveles de precipitación acumulada y un aumento de ésta al aumentar la altitud asociada a la clases de deshielo, y por tanto, al tiempo de lixiviación de las aguas, la clase $\mathrm{Ar} / \mathrm{V}$ tiene similar promedio que la clase $\mathrm{Ar} / \mathrm{Es}$. En este caso se esperaba que la clase $\mathrm{Ar} / \mathrm{V}$ tuviese menor valor promedio que $\mathrm{Ar} /$ Es dado el origen del flujo distinto entre las dos. Sin embargo, debido a su pertenencia a climas áridos, predomina el alto nivel de evaporación, con lo cual aumenta el nivel de sales disueltas en las aguas (Drever 2002).

A medida que las clases se asocian a climas con mayores niveles de precipitación, la $\mathrm{CE}$ disminuye $(\mathrm{L}, \mathrm{V})$ producto del alto caudal de dilución y del alto lavado del suelo existente (Drever 2002, Alan \& Castillo 2007) y aumentan en clases provenientes del deshielo debido a que este caudal de dilución cobra menor importancia a medida que aumentan los niveles de precipitación.

De igual forma, la CE de acuerdo a la composición litológica media de la cuenca se ve influenciada por los dos factores anteriores, y por tanto, existen clases con similares valores promedios de CE a pesar de su diferencia en la composición litológica de la subcuenca.

La alta influencia del factor Clima, así como de los factores Origen de Flujo y Geología pareciera indicar que la calidad geoquímica de las aguas en Chile está influenciada por los niveles de precipitación, evaporación, topografía y litología de la subcuenca y de las subcuencas aguas arriba. 
La interpretación de las clases REC-Chile puede hacerse en forma conjunta en donde es posible considerar el efecto combinado, tanto del patrón hidrológico como el de la calidad geoquímica. Así, para un tramo de río, la clasificación a un tercer nivel permite interpretar la magnitud del caudal anual medio su estacionalidad y valores medios de conductividad eléctrica.

La evaluación de las clasificaciones a priori, como la REC, es necesaria no solo para cuantificar el poder de discriminación de las clasificación sobre de la variabilidad de los objetos, sino también para determinar si la elección de los factores controladores y/o su división en las categorías propuestas es correcta.

Considerando la escala espacial de los factores perteneciente a estos dos esquemas y al porcentaje de clases representadas en la evaluación, se puede concluir que el esquema $\mathrm{CL}$ es un buen esquema cuando se requiere clasificar los ríos de Chile a una macroescala bajo el objetivo de clasificación de magnitud de caudales medios.

Estas correspondencias entre las variables del hidrograma y las clases RECChile permiten realizar una interpretación descriptiva del hidrograma medio anual. Esta interpretación sumada a la posibilidad de extrapolación a tramos de ríos en cuencas con escasa o nula información dotan a la REC-Chile de numerosos usos en la gestión de cuencas, por ejemplo, considerando que la REC-Chile es una clasificación de ríos en condiciones de régimen natural (Peredo-Parada et al. 2010), es posible determinar el nivel de alteración hidrológica que presentan los ríos de Chile comparando el régimen 
Capítulo 3 EVAluación de LA REC-Chile hidrológico actual de un tramo de río con el hidrograma medio anual en condiciones naturales correspondiente a ese tramo de río entregado por la REC-Chile. Asimismo, permite comparar y definir un ranking de alteración hidrológica entre distintos tramos de río pertenecientes a la misma clase (Detenbeck et al. 2000)

Asumiendo que la hidrología controla los procesos ecológicos en los ecosistemas acuáticos, es posible también utilizar la REC-Chile como plataforma en la gestión de los ecosistemas fluviales. Evaluando diversos índices que son asociados a la calidad del agua, es posible comparar el estado ecológico de los tramos de río que pertenecen a una misma clase (Detenbeck et al. 2000, Hawkins et al. 2000) y posteriormente determinar tramos de referencias en condiciones prístinas, lo cual es el comienzo para la formulación de planes de restauración fluvial. 


\subsection{CONCLUSIONES}

REC-Chile es una clasificación a priori, geográficamente independiente, basada en una superposición de factores controladores. Estas características dotan a la REC-Chile de ductilidad y flexibilidad para trabajar a varias escalas espaciales y bajo diferentes objetivos de clasificación, además permite extrapolar características de hidrograma a tramos de río en cuencas sin información las cuales son útiles para implementar en zonas con poca información hidrológica.

Los resultados del test de clasificación son satisfactorios, lo cual permite mostrar que la elección de los factores controladores y sus categorías parecieran ser adecuados para discriminar el patrón hidrológico y la calidad geoquímica de las aguas en los ríos de Chile. El esquema de clasificación CLPR, dado por la combinación de los factores controladores Clima y Posición Relativa de la Subsubcuenca, pareciera ser adecuado para discriminar la variación espacial del caudal medio anual y la magnitud de sus variaciones estacionales. Asimismo, el esquema de clasificación CLOF, dado por la combinación de los factores Clima y Origen de Flujo, pareciera serlo para discriminar la estacionalidad del hidrograma medio anual. Como también la superposición jerárquica de los factores Clima, Origen de Flujo y Geología lo es para discriminar adecuadamente la variación espacial geoquímica de los ríos de Chile

Los resultados del AC permiten realizar una interpretación basada en la magnitud del caudal medio anual y su estacionalidad de las clases de los 
CAPÍTUlo 3 EVALUACIÓN DE LA REC-CHILE esquemas anteriores, permitiendo desarrollar un hidrograma descriptivo para todos los tramos de río en condiciones naturales. Un análisis de estadísticos básicos permitió asociar valores de CE a diversas clases del tercer nivel de clasificación.

Las diversas propiedades y usos de la REC-Chile permiten que ésta sea una plataforma para la gestión y conservación de cuencas y ecosistemas acuáticos en los ríos de Chile. 


\subsection{BIBLIOGRAFÍA}

ALLAN D \& CASTILLO M. 2007. Stream ecology. Structure and function of running waters. $2^{\text {nd }}$ edition. Springer Dordrecht, the Netherlands. $436 \mathrm{pp}$.

BAILEY RG, RD PFISTER \& JA HENDERSON. 1978. Nature of Land and Resource Classification. A review. Journal of Forestry 76: 650-655.

BRYAN B. 2006. Synergistic techniques for better understanding and classifying the environmental structure of landscape. Environmental Management 37(1): 126140 .

COMISION EUROPEA. 2000. Directive 2000/60/EC of the European Parliament of the Council of 23 October 2000 establishing a framework for Community action in the field of water policy. OJEC (Official Journal of the European Communities) 22.12.2000. $72 \mathrm{pp}$.

DETENBECK N, S BATTERMAN, V BRADY, J BRAZNER, V SNARSKI, D TAYLOR, J THOMPSON \& J ARTHUR. 2000. A Test of watershed classification systems for ecological risk assessment. Environmental Toxicology and Chemistry 19(4): 1174-1181.

DREVER J. 2002. The Geochemistry of Natural Waters. Surface and Groundwater Environments. Prentice Hall. Third Edition. New Jersey. ISBN: 0-13-272790-0. $436 \mathrm{pp}$.

DGA (2004). Diagnóstico y clasificación de los cuerpos de agua según objetivos de calidad. Informe Técnico. CADE IDEPE.

FERRANDO F. 1992-1993. Clasificación Hidrodinámica de Chile. Cuadernos I. Geográfica. 18-19: 57-74.

GREENACRE M \& O NENADIC. 2007. Simple, Multiple and Joint Correspondence Analysis. $\mathrm{R}$ package version 10.21. URL http://CRAN.Rproject.org/

GREENACRE M. 2008. La práctica del análisis de correspondencia. ISBN 97884-96515-71-0. Eds. Fundación Banco Bilbao Vizcaya Argentaria. 351pp. www.ffbbva.es

GONZÁlEZ DEL TÁNAGO M \& D GARCÍA DE JALÓN. 2006. Propuesta de caracterización jerárquica de los ríos españoles para su clasificación según la Directiva Marco de la Unión Europea. Limnética 25 (3): 693-712. 
Capítulo 3 Evaluación de LA REC-ChILE HARRIS N, A GURNELL, D HANNAH \& G PETTS. 2000. Classification of rivers regimes: a context for hydroecology. Hydrological Process. 14: 2831-2848.

HAWKINS C, R NORRIS, J GERRITSEN, R HUGHES, S JACKSON, R JOHNSON \& J STEVENSON. 2000. Evaluation of the use of landscape classifications for the prediction of freshwater biota: synthesis and recommendations. Journal of American Benthological Society. 19 (3): 541-556.

HOPKINS II RL \& BM BURR. 2009. Modelling freshwater fish distributions using multiscale landscape data: A case study of six narrow range endemics. Ecological Modelling 220, 2024-2034.

NAIMAN RJ. 1998. Biotic stream classification. Pages 97-119 in R.J. Naiman and R.E. Bilby (Editors). River Ecology and Management: Lessons from the Pacific Coastal Ecoregion. Springer-Verlag, New York.

OMERNIK J. 1987. Ecoregions of the conterminous United States. Annals of the Association of American Geographers 77: 118-125.

OMERNIK J \& R BAILEY. 1997. Distinguish between watersheds and ecoregions. Journal of the American Water Resources Association. 33 (5): 935949 .

PEREDO-PARADA $\mathrm{M}, \mathrm{F}$ MARTÍNEZ-CAPEL, D QUEVEDO \& A HERNÁNDEZ-MASCARELL. 2010. Implementation of an Eco-Hydrological Classification in Chilean Rivers. Gayana. En prensa.

R DEVELOPMENT CORE TEAM. 2008. R: A language and environment for statistical computing. R Foundation for Statistical Computing, Vienna, Austria. ISBN 3-900051-07-0, URL http://www.R-project.org.

RONCHAIL J. 1989. Advecciones polares en Bolivia: Caracterización de los efectos climáticos. Bulletin. Inst. Fr. Et. And. 18 (1): 65-73.

SNELDER T \& B BIGGS. 2002. Multiscale River Enviroment Classification for Water Resources Management. American Water Resources Association 38 (5): 1225-1239.

SNELDER T, B BIGGS \& R WOODS. 2005. Improved Eco-Hydrological Classificaction of Rivers. River Research and Applications. 21:609-628.

SNELDER T, N LAMOUROUX, J LEATHWICK, H PELLA, E SAQUET \& U SHANKAR. 2009. Predictive mapping of the natural flow regimes of France. Journal of Hydrology 373: 57-67. 
Capítulo 3 Evaluación DE LA REC-CHILE

VANNOTE RL, GW MINSHALL1, KW CUMMINS, JR SEDELL \& CE CUSHING. 1980. The River Continuum Concept. Canadian Journal of Fisheries and Aquatic Science. 37: 130-137.

VAN SICKLE J. 1997. Using mean similarity dendrograms to evaluate classifications. Journal of agricultural, Biological and Environmental Statistics 2: 370-388.

VAN SICKLE J \& R HUGHES. 2000. Classification strengths of ecorregions, catchments, and geographic cluster for aquatic vertebrates in Oregon. Journal of North American Benthological Society 19 (3): 370-384.

VILA I. \& X MOLINA. 2006. Capítulo 3: Variables químicas y biológicas. En: Manual de Calidad del Agua. Servicio Agrícola y Ganadero. (Eds. Molina X. \& I. Vila). Santiago 106 pp 


\section{Capitula 4}

Base de datos Eco-Hidrológica de los ríos de Chile: una herramienta

de gestión para los ecosistemas acuáticos 



\subsection{RESUMEN}

El conocimiento de las especies nativas de Chile se caracteriza por ser insuficiente, incompleto, fragmentado y poco actualizado, lo cual constituye una de las principales limitantes para desarrollar estrategias de conservación de especies o planes de conservación y recuperación de sistemas acuáticos. Lo anterior cobra mayor importancia dada la mayor presión que tienen actualmente estos ecosistemas en el país. Para paliar este vacío se ha confeccionado una Base de Datos Eco-Hidrológica de los ríos de Chile. Esta base de datos cuenta con información hidrológica, fisicoquímica de las aguas, sedimentológicas e información íctica. Esta última información se ha basado en recopilación bibliográfica de literatura referente a distribución de peces, dividida en tres niveles (I, II y III) de acuerdo al detalle y escala espacial de la información (macroescala, mesoescala y microescala, respectivamente). La Base de Datos Eco-Hidrológica confeccionada la conforman 393 estaciones fluviométricas, 361 estaciones de calidad del agua, 92 estaciones sedimentológicas y un total de 1580 registros de peces distribuidos a lo largo de todo Chile. Esta base de datos se ha integrado en la plataforma de gestión Clasificación Eco-Hidrológica de los ríos de Chile (REC-Chile), la cual se construyó basándose en los factores medioambientales que controlan el régimen hídrico de un río. La utilización conjunta de la base de datos junto con REC-Chile permitirá desarrollar una mejor planificación y gestión de los ecosistemas acuáticos en Chile. 


\subsection{INTRODUCCIÓN}

Las características hidrológicas y morfológicas de Chile han sido los factores claves en la evolución de los peces dulceacuícolas de Chile (Vila et al. 1999). El aislamiento biogeográfico y las características hidrológicas de los ríos son los principales factores que justifican la baja riqueza específica y el alto endemismo junto con la tendencia generalizada a poseer tamaños pequeños (Habit et al. 2006, Vila et al. 2006a, Dyer 2000a, Vila et al. 1999).

En Chile, la fauna íctica nativa está comprendida por 11 familias, 17 géneros y 44 especies (Habit et al. 2006, Dyer 2000a). Los grupos más representativos corresponden a los órdenes Siluriformes (11 especies), Osmeriformes (9 especies), Atheriniformes ( 7 especies). También se representan en menor grado los Petromyzontiformes (2 especies), Characiformes (4 especies), Cyprinodontiformes (6 especies), Perciformes (4 especies) y Mugiliformes (1 especie).

La mayor riqueza de especies nativas se encuentra en la zona centro-sur del país entre los ríos Maule e Imperial (Dyer 2000a). El río Biobío es aquél que posee la mayor diversidad íctica con 18 especies nativas (Dyer 2000a, Vila et al. 1999). El extremo norte del país presenta un alto índice de endemismo, con especies que sólo habitan en una cuenca determinada (Dyer 2000a, Vila et al. 1999). 
CAPÍTULO 4 BASE DE DATOS ECO-HIDROLÓGICA. A la riqueza íctica nativa del país hay que añadir 22 especies exóticas, de las cuales sólo dos (Cheirodon interruptus y Odontesthes bonaerensis) están emparentadas a nivel de familia con alguna especie chilena (Dyer 2000a). Entre las especies exóticas, la trucha arcoiris (Oncorhynchus mykiss), trucha café o común (Salmo trutta), salmón del atlántico (Salmo salar) y carpa (Ciprinius carpio) son las más importantes desde el punto de visto económico y de distribución en el país, destacando en el extremo sur del país el predominio de la familia exótica Salmonidae debido a su alto nivel de adaptación a las aguas frías del sur de Chile (Soto et al. 2006).

El estado de conservación de los peces nativos de Chile presenta grandes problemas, ya que solo trece especies están consideradas "fuera de peligro". Los taxa con mayor número de especies con problemas en nivel de "peligro de extinción" son los Siluriformes (7 especies) y Cyprinodontiforme (4 especies). Estos dos órdenes coinciden con los de mayor endemismo en cuanto a número de especies y a su limitada distribución geográfica (Habit et al. 2006 Dyer 2000a, Vila et al. 1999).

Dentro de las principales causas del deteriorado estado de conservación de los peces dulceacuícolas de Chile, está la modificación del régimen hidrológico debido a canalizaciones, represamiento de los ríos (Vila et al. 1999) y extracción de agua para riego (Habit et al. 2006), lo cual se traduce en la destrucción del hábitat físico de las especies. La alteración de la calidad fisicoquímica del agua, es otra causa importante del deterioro del hábitat (Vila et al. 1999). Las acciones más comunes son vertidos de residuos industriales líquidos y aguas residuales, extracción de áridos, sustitución de bosque nativo por bosque forestal y contaminación difusa por 
CAPITULO 4 BASE de DATOS ECO-HIDROLÓGICA.

pesticidas (Habit et al. 2006). Y finalmente, la acción de las especies introducidas en los inicios del siglo XX, principalmente en la zona centrosur de Chile.

Por otro lado, el conocimiento que se tiene de las distintas especies nativas en relación a su distribución, abundancia o densidad, preferencia de hábitat y biología poblacional y reproductiva, es escaso (Habit et al. 2006), incompleto, fragmentado y poco actualizado (Vila 2001). Dicha información está en relación a Siluriformes (Scott et al. 2007, Pardo et al. 2005, Habit 2005, Habit et al. 2003, Pardo 2002, Arratia 1983), Osmeriformes (Cussac et al. 2004, Murillo \& Ruiz 2002, Peredo \& Sobarzo 1994), Atheriniformes (Dyer 2000b, Comte \& Vila 1987, Bahamondes et al. 1979), Perciformes (Habit \& Belk 2007, Habit 1998), Cyprinodontiformes (Vila et al. 2006b, Vila 2006). Recopilación de los estudios realizados a la fecha pueden ser revisados en Habit et al. (2006), Vila et al. (2006a) y Ruiz et al. (2006). Una completa lista sistemática de las especies puede ser revisada en Dyer (2000a) y en Vila et al. (2006a).

De acuerdo a lo anterior, el escaso conocimiento existente a la fecha no permite avanzar en políticas o gestión para la conservación y/o rehabilitación de ecosistemas acuáticos, como por ejemplo, en el uso de metodologías de simulación del hábitat físico para la determinación del régimen ambiental de caudales (Benetti et al. 2004, Tharme 2003); en la elaboración de directrices en proyectos de rehabilitación fluvial (Bond \& Lake 2003) y en el desarrollo de medidas de mitigación y planes de conservación de las especies dulceacuícolas de Chile (Habit et al. 2006). 
CAPÍTULO 4 BASE DE DATOS ECO-HIDROLÓGICA. Como una medida para paliar estos vacíos de información y avanzar en políticas de conservación y rehabilitación de ecosistemas acuáticos, se ha confeccionado una base de datos acoplada a la plataforma de gestión "Clasificación Eco-Hidrológica de los ríos de Chile" (REC-Chile) (PeredoParada et al. 2010). La plataforma de gestión REC-Chile es la adaptación a las condiciones ambientales de Chile de la herramienta River Environment Classification (REC) desarrollada por Snelder \& Biggs (2002). La utilización conjunta de un sistema de clasificación o zonificación, como REC-Chile, y una base de datos de peces constituyen la base para la determinación del estado ecológico de los ríos basado en índices ícticos (Noble et al. 2007, Schmutz et al. 2007), entre otros.

Dentro de las potencialidades de uso que permite la REC están aquellas destinadas al manejo del recurso hídrico a nivel de cuenca (Snelder \& Huguey 2005), a la caracterización fisicoquímica de los ríos (Snelder et al. 2004a), a la conservación de ecosistemas acuáticos y a la planificación y gestión medioambiental (Peredo-Parada et al. 2009, Snelder et al. 2004b).

La Clasificación Eco-Hidrológica de los ríos de Chile, es una herramienta que consiste en la superposición jerárquica de las variables o factores ambientales que son los causantes principales de la variación espacial del patrón hidrológico en un río (Snelder et al. 2005, Snelder \& Biggs 2002). Entre los factores que controlan este patrón hidrológico en Chile están el clima, la topografía y la geología (Peredo-Parada et al. 2010). De estas variables se definieron los factores controladores aplicables a Chile, los cuales resultaron ser: Clima, Origen de flujo (que engloba las variables pertenecientes a altitud, glaciares, lagos), Geología, Posición relativa de 
CAPITULO 4 BASE DE DATOS ECO-HIDROLÓGICA.

subsubcuenca (definiendo zonas de cabecera, tramos medio y alto $\mathrm{y}$ desembocadura) Uso de suelo y Pendiente del tramo (Peredo-Parada et al. 2010).

Los factores controladores que influyen sobre la magnitud y estacionalidad del flujo son el Clima, Origen de Flujo y Posición relativa de subsubcuenca. Aquellos que tienen mayor influencia sobre la calidad de las aguas son los factores Geología y Uso de suelo, mientras que el factor Pendiente del tramo influye sobre las condiciones hidráulicas (Snelder et al. 2005, Snelder \& Biggs 2002, Peredo-Parada et al. 2010).

El resultado de la combinación de estos factores controladores es una clasificación única para cada tramo de río, el cual permite ser caracterizado de acuerdo a variables hidrológicas, fisicoquímicas o una combinación de éstas. Los resultados de la aplicación de la REC en Chile han sido satisfactorios pudiendo caracterizar los tramos de ríos de acuerdo al régimen natural hidrológico, tanto en magnitud como en estacionalidad del hidrograma. (Peredo-Parada et al. 2010).

En relación a lo anterior, el objetivo del presente trabajo ha sido confeccionar una base de datos georreferenciada que integre información íctica, hidrológica y fisicoquímica de los ríos de Chile, la cual en conjunto con la REC-Chile o algún sistema de clasificación alternativo, puedan servir de base para planificar y gestionar el ecosistema acuático de los ríos de Chile. 


\subsection{METODOLOGÍA}

La base de datos se generó a partir de dos grandes bloques de información.

El primer bloque corresponde a información gubernamental proporcionada por la Dirección General de Aguas (DGA) del Ministerio de Obras Públicas (MOP), relativas a fluviometría, calidad del agua y sedimentología. El segundo bloque es información íctica obtenida a partir de recopilación bibliográfica de publicaciones científicas e informes públicos generados de diversos proyectos. En la selección de la literatura se ha dado preferencia a aquella que incluye información de especies nativas.

\subsubsection{Información gubernamental.}

La información fluviométrica corresponde a la información de cada estación fluviométrica existente en Chile: Coordenadas UTM, código BNA (código oficial de identificación de la estación), y códigos de cuenca, subcuenca y subsubcuenca en la cual se sitúan. Análogamente, la información fisicoquímica es la información de cada estación de calidad de aguas: coordenadas UTM, código BNA, parámetros fisicoquímicos que se miden en cada una de ellas y la frecuencia a la cual son medidos dichos parámetros. Y por último, para cada estación sedimentológica se dispone de las coordenadas UTM y código BNA. 
CAPITULO 4 BASE de dATOS ECO-HIDRológICA.

\subsubsection{Información de fauna íctica.}

Dada la heterogeneidad en la escala espacial de la información entregada en la bibliografía especializada e informes de proyectos públicos, se definieron tres niveles (I, II, III) de información de fauna íctica, de acuerdo al detalle de información por parte del autor y a la escala espacial de ésta.

La información del Nivel I se define a una escala espacial macro $\left(10^{5}\right.$ a $10^{3}$ $\mathrm{km}^{2}$ ). Este nivel de información corresponde a aquel en donde el autor señala distribuciones geográficas de peces que abarcan cuencas e incluso regiones. Aquí se incluyen campos con información de la especie, su rango de distribución, autor y fecha de la publicación.

La información del Nivel II comprende información a nivel de mesoescala, teniendo como escala espacial desde la cuenca hasta un tramo local de río $\left(10^{3}\right.$ a $\left.10^{1} \mathrm{~km}^{2}\right)$. Se ha incluido en este nivel aquella información señalada por el autor(es) referente al río, tramo y/o localidad en donde se practicó el muestreo. Sin embargo, el autor(es) no especifica mayores detalles sobre el tipo de muestreo utilizado, resultados cuantitativos del muestreo, fecha de realización de éste, entre otros. Los campos utilizados para este nivel contienen información de la especie, fecha de muestreo, año y autor de la publicación, código de la cuenca, subcuenca y/o subsubcuenca de distribución.

La información del Nivel III es una información más detallada actuando a nivel de microescala. El autor(es) señala las coordenadas de la estación de muestreo, fecha de realización, tipo de muestreo y resultados, así como 
CAPÍTULO 4 BASE DE DATOS ECO-HIDROLÓGICA. también puede indicar valores de parámetros fisicoquímicos del agua. Para este nivel de información se incluyen campos con información sobre la especie, autor y año de publicación, lugar de muestreo, características del muestreo, parámetros fisicoquímicos del agua y código de cuenca, subcuenca y subsubcuenca.

Para todos los niveles de información señalados anteriormente, se incluyeron campos sobre el código de cuenca, subcuenca y subsubcuenca. Estos códigos son los oficiales asignados por la DGA, permitiendo así que la base de datos pueda estar conectada con la REC-Chile y con información oficial de la DGA.

La totalidad de la información recopilada (fluviométrica, fisicoquímica, sedimentológica e íctica) se implementó en un Sistema de Información Geográfico, con sistemas de coordenadas PSAD 1956 UTM Huso 18S. 


\subsection{RESULTADOS}

\subsubsection{Información gubernamental}

La base de datos obtenida cuenta con 393 estaciones fluviométricas, 361 estaciones de calidad del agua, 62 estaciones sedimentológicas. Además cuenta con 124 registros de información Nivel I, 450 registros de información Nivel II y con 171 estaciones de muestreo específicos de colecta de peces con 1010 registros pertenecientes a la información Nivel III.

La información hidrológica se obtuvo a partir de 393 estaciones fluviométricas. Actualmente sólo 321 están vigentes (Tabla 4.1). Las estaciones fluviométricas que no están vigentes se han incluido en la base de datos, ya que permite realizar un análisis suplementario en aquellos años donde la estación estaba en vigencia. Las estaciones de calidad de aguas y sedimentológicas también se obtuvieron de la información entregada por la DGA con 361 y 62 estaciones respectivamente. Para ambos casos se incluyeron las estaciones suspendidas, (Tabla 4.1).

\subsubsection{Información íctica}

La información Nivel I contiene 120 registros abarcando la totalidad de las especies ícticas nativas del país. La distribución geográfica de esta información abarca todo Chile, desde la cuenca del río Lluta $\left(18^{\circ} \mathrm{S}\right)$ hasta la 
CAPÍTULO 4 BASE DE DATOS ECO-HIDROLÓGICA. Tierra del Fuego $\left(55^{\circ} \mathrm{S}\right)$. En este nivel de información se indican las cuencas, y sus códigos asociados, de los extremos norte y sur de distribución definiendo así el rango de distribución para cada especie.

\begin{tabular}{|c|c|c|c|c|c|c|}
\hline \multirow[t]{2}{*}{ Región } & \multicolumn{2}{|c|}{$\begin{array}{l}\mathbf{N}^{0} \text { Estaciones } \\
\text { fluviométricas }\end{array}$} & \multicolumn{2}{|c|}{$\begin{array}{l}N^{0} \text { Estaciones } \\
\text { fisicoquímicas }\end{array}$} & \multicolumn{2}{|c|}{$\begin{array}{c}\mathrm{N}^{0} \text { Estaciones } \\
\text { sedimentológicas }\end{array}$} \\
\hline & Vig. & Susp. & Vig. & Susp. & Vig. & Susp. \\
\hline I Región & 5 & 0 & 4 & 0 & 0 & 0 \\
\hline II Región & 33 & 15 & 21 & 37 & 3 & 0 \\
\hline III Región & 29 & 0 & 28 & 0 & 3 & 0 \\
\hline IV Región & 46 & 3 & 54 & 0 & 6 & 7 \\
\hline V Región y RM & 41 & 0 & 68 & 0 & 12 & 2 \\
\hline VI Región & 10 & 0 & 24 & 0 & 1 & 0 \\
\hline VII Región & 36 & 41 & 27 & 0 & 5 & 0 \\
\hline VIII Región & 47 & 0 & 22 & 0 & 10 & 2 \\
\hline IX Región & 29 & 0 & 18 & 0 & 8 & 0 \\
\hline X Región & 45 & 16 & 25 & 33 & 2 & 1 \\
\hline Total & 321 & 72 & 291 & 70 & 50 & 12 \\
\hline
\end{tabular}

La información Nivel II contiene 450 registros abarcando 37 especies nativas (25 spp. endémicas, 12 spp. nativas) y 6 especies introducidas. Dentro de las especies nativas con más número de registros están Trichomycterus areolatus (56 registros), Galaxias maculatus (39 registros), Basilichthys australis (27 registros) y Percichthys trucha (27 registros) (Tabla 4.2). Dentro de las introducidas la especie O. mykiss con 83 registros es la especie con mayor abundancia. El rango geográfico de este tipo de información va desde del extremo norte de Chile $\left(18^{\circ} \mathrm{S}\right)$ hasta la zona de Aysén $\left(47^{\circ} \mathrm{S}\right)$. 
La información Nivel III contiene 171 estaciones de muestreo de peces con 1010 registros correspondientes a 34 especies nativas (7 spp. nativas y 27 spp. endémicas) y 8 especies introducidas. Dentro de las especies nativas con mayor número de registros se encuentran T. areolatus (128 registros), P. gillissi (80 registros), G. maculatus (67 registros) y B. australis con 65 registros (Tabla 2.1). En las especies introducidas aquellas con mayor cantidad de registros son $O$. mykiss (113 registros) y S. trutta (94 registros). En este nivel de información se han incluido los puntos de muestreo en los cuales no se han capturado peces ni nativos ni introducidos.

De la Tabla 4.2 se observa que las especies T.areolatus, G. maculatus, $P$. gillissi, B. australis y $P$. trucha, son las especies que mayor cantidad de registros tienen al sumar ambos niveles de información (II y III). Las especies distribuidas en la zona norte de Chile, (O. agassii, O. ascotanensis, O. chungaraensis, O. laucaensis, O. parinacotensis, O. piacotensis, $T$. chungaraensis, T. laucaensis y B. semotilus) tienen una escasa cantidad de registros en ambos niveles de información. Bajo esta misma situación de baja cantidad de registros se encuentran las especies D. chilensis, G. globiceps, H. macrei, M. lapicida y O. brevianalis. Además existen especies que no tienen información de Nivel II ni tampoco de Nivel III, como son: $B$. gothei, C. killiani, G. alpinus, O. itatanum y O. hatcheri. 
CAPÍTULO 4 BASE DE DATOS ECO-HIDROLÓGICA.

Tabla 4.2. Rango geográfico de cuencas vertientes (límites norte-sur) en que cada especie nativa fue localizada, y números de registros (entre paréntesis) según fuese su nivel de información (II y III).

\begin{tabular}{|c|c|c|}
\hline \multirow{2}{*}{ Especie } & Nivel II & Nivel III \\
\hline & Distribución & Distribución \\
\hline Aplochiton marinus? Eigenmann 1928 & $\mathrm{~S} / \mathrm{I}$ & $\mathrm{S} / \mathrm{I}$ \\
\hline Aplochiton taeniatus Jenyns 1842 & Chiloé-Aysén (12) & $\mathrm{S} / \mathrm{I}$ \\
\hline Aplochiton zebra Jenyns 1842 & Biobío-Maullín (16) & Itata (1) \\
\hline Basilichthys australis Eigenmann 1928 & $\begin{array}{l}\text { Aconcagua-Chiloé } \\
\text { (27) }\end{array}$ & $\begin{array}{l}\text { Aconcagua-Valdivia } \\
\text { (65) }\end{array}$ \\
\hline Basilichthys cf. semotilus Cope 1874 & $\mathrm{~S} / \mathrm{I}$ & Loa $(2)$ \\
\hline $\begin{array}{l}\text { Basilichthys microlepidotus Jenyns } \\
1841\end{array}$ & $\begin{array}{l}\text { Huasco-Aconcagua } \\
\text { (4) }\end{array}$ & Huasco-Choapa (11) \\
\hline Brachygalaxias bullocki Regan 1908 & Biobío-Chiloé (6) & Itata-Valdivia (9) \\
\hline Brachygalaxias gothei Busse 1982 & $\mathrm{~S} / \mathrm{I}$ & $\mathrm{S} / \mathrm{I}$ \\
\hline Bullockia maldonadoi Eiegnmann 1928 & Andalién-Biobío (5) & Andalién-Toltén (36) \\
\hline Cheirodon australe Eigenmann 1928 & Valdivia-Chiloé (3) & Maule-Valdivia (16) \\
\hline Cheirodon galusdae Eigenmann 1928 & Biobío (5) & Maule-Toltén (51) \\
\hline Cheirodon killiani Campos 1982? & $\mathrm{S} / \mathrm{I}$ & $\mathrm{S} / \mathrm{I}$ \\
\hline Cheirodon pisciculus Girard 1855 & Huasco-Biobío (10) & Maipo-Maule (9) \\
\hline Diplomystes camposensis Arratia 1987 & Biobío-Valdivia (2) & Imperial -Valdivia (28) \\
\hline Diplomystes chilensis Molina 1782 & Valdivia (1) & Rapel e Imperial (6) \\
\hline $\begin{array}{l}\text { Diplomystes nahuelbutaensis Arratia } \\
1987\end{array}$ & Biobío (6) & Maule-Biobío (9) \\
\hline Galaxias alpinus Jenyns 1842 & $\mathrm{~S} / \mathrm{I}$ & $\mathrm{S} / \mathrm{I}$ \\
\hline Galaxias globiceps Eigenmann 1928 & Maullín-Chiloé (2) & $\mathrm{S} / \mathrm{I}$ \\
\hline Galaxias maculatus Jenyns 1842 & Huasco-Aysén (39) & Itata-Valdivia (67) \\
\hline Galaxias platei Steindachner 1898 & Valdivia-Aysén (11) & Valdivia-Bueno (8) \\
\hline Geotria australis Gray 1851 & Biobío-Aysén (7) & Andalién-Valdivia (38) \\
\hline Hatcheria macrei Girard 1855 & Aysén (2) & $\mathrm{S} / \mathrm{I}$ \\
\hline Mordacia lapicida Gray 1851 & Biobío (1) & Andalién (7) \\
\hline Mugil cephalus Linnaeus 1758 & Huasco-Valdivia (5) & Andalién (8) \\
\hline Nematogenys inermis Guichenot 1848 & Aconcagua-Biobío (7) & Andalién-Imperial (16) \\
\hline $\begin{array}{l}\text { Odontesthes (Cauque) breavianalis } \\
\text { Günther } 1880\end{array}$ & Choapa-Biobío (3) & $\mathrm{S} / \mathrm{I}$ \\
\hline $\begin{array}{l}\text { Odontesthes (Cauque) } \\
\text { Steindachne } 1896\end{array}$ & $\mathrm{~S} / \mathrm{I}$ & $\mathrm{S} / \mathrm{I}$ \\
\hline $\begin{array}{l}\text { Odontesthes (Cauque) mauleanum } \\
\text { Steindachne } 1896\end{array}$ & Biobío-Bueno (14) & Itata-Andalién (3) \\
\hline
\end{tabular}


CAPÍtulo 4 BAse de dATOS ECo-HidRolóGicA.

Tabla 4.2. Rango geográfico de cuencas vertientes (límites norte-sur) en que cada especie nativa fue localizada, y números de registros (entre paréntesis) según fuese su nivel de información (II y III).

\begin{tabular}{|c|c|c|}
\hline \multirow{2}{*}{ Especie } & Nivel II & \multirow{2}{*}{$\begin{array}{c}\text { Nivel III } \\
\text { Distribución }\end{array}$} \\
\hline & Distribución & \\
\hline Odontesthes hatcheri Eigenmann 1909 & $\mathrm{~S} / \mathrm{I}$ & $\mathrm{S} / \mathrm{I}$ \\
\hline Orestias agassi Valenciennes 1846 & Salar Huasco (1) & $\begin{array}{l}\text { Salar Huasco, Isluga } \\
\text { (3) }\end{array}$ \\
\hline Orestias ascotanensis Parenti 1984 & Salar Ascotán (1) & $\begin{array}{l}\text { S. Ascotán y Carcote } \\
\text { (3) }\end{array}$ \\
\hline $\begin{array}{l}\text { Orestias chungaraensis Vila \& Pinto } \\
1986\end{array}$ & Lago Chungará (1) & Lago Chungará (1) \\
\hline Orestias laucaensis Arratia 1982 & Lauca (1) & Río Lauca (1) \\
\hline Orestias parinacotensis Arratia 1982 & Parinacota (1) & Parinacota (1) \\
\hline Orestias piacotensis Vila 2006 & Piacota (1) & Piacota (1) \\
\hline Percichthys melanops Girard 1855 & $\begin{array}{l}\text { Aconcagua-Valdivia } \\
\text { (5) }\end{array}$ & Mataquito-Andalién (7) \\
\hline Percichthys trucha Valenciennes 1833 & $\begin{array}{l}\text { Aconcagua-Bueno } \\
(22)\end{array}$ & $\begin{array}{l}\text { Mataquito-Valdivia } \\
(60)\end{array}$ \\
\hline Percilia gillissi Girard 1855 & Maipo-Valdivia (16) & $\begin{array}{l}\text { Mataquito-Valdivia } \\
(80)\end{array}$ \\
\hline Percilia irwini Eigenmann 1928 & Biobío (8) & Maule-Biobío (44) \\
\hline $\begin{array}{l}\text { Trichomycterus areolatus Valenciennes } \\
1840\end{array}$ & Huasco-Chiloé (56) & $\begin{array}{l}\text { Aconcagua-Bueno } \\
\text { (128) }\end{array}$ \\
\hline $\begin{array}{l}\text { Trichomycterus chiltoni Eigenmann } \\
1928\end{array}$ & Aconcagua-Biobío (5) & Maule-Biobío (9) \\
\hline $\begin{array}{l}\text { Trichomycterus chungaraensis Arratia } \\
1983\end{array}$ & Lago Chungará (1) & Lago Chungará (1) \\
\hline $\begin{array}{l}\text { Trichomycterus laucaensis Arratia } \\
1983\end{array}$ & Lauca (1) & Lauca y Parinacota (2) \\
\hline $\begin{array}{l}\text { Trichomycterus rivulatus Valenciennes } \\
1840\end{array}$ & Salar Huasco (1) & Altiplánicas (4) \\
\hline
\end{tabular}

S/I: Sin Información

La distribución geográfica de las estaciones de muestreo de peces recopilada a nivel de regiones, señalando cuencas involucradas, rango de fecha en el cual se realizaron los muestreos y las referencias de éste se muestran en la Tabla 4.3. De ésta se destaca la heterogeneidad en la densidad de estaciones por muestreo en cada región, existiendo zonas de Chile con mayor densidad (entre las cuencas Rapel e Imperial) y otras con menor densidad (entre las 
CAPÍTULO 4 BASE DE DATOS ECO-HIDROLÓGICA. cuencas Loa y Choapa). Al sur de la cuenca del río Bueno no hay estaciones de muestreo.

\begin{tabular}{|c|c|c|c|c|}
\hline Región & Cuenca & $\begin{array}{c}\mathrm{N}^{0} \\
\text { estac. }\end{array}$ & $\begin{array}{c}\text { Fechas } \\
\text { muestreos }\end{array}$ & Referencias \\
\hline \multirow{2}{*}{$\begin{array}{l}\text { Región I de } \\
\text { Tarapacá }\end{array}$} & Altiplánica & 8 & 2006 & U. de Chile Lab. Limnología \\
\hline & $\begin{array}{l}\text { Qbda. } \\
\text { Camarones }\end{array}$ & 1 & 2006 & U. de Chile Lab. Limnología \\
\hline \multirow{2}{*}{$\begin{array}{l}\text { Región II de } \\
\text { Antofagasta }\end{array}$} & Río Loa & 4 & 2006 & U. de Chile Lab. Limnología \\
\hline & Salar Michinca & 2 & 2006 & U. de Chile Lab. Limnología \\
\hline \multirow{2}{*}{$\begin{array}{l}\text { Región III de } \\
\text { Copiapó }\end{array}$} & Río Copiapó & 2 & 2006 & U. de Chile Lab. Limnología \\
\hline & Río Huasco & 4 & 2006 & U. de Chile Lab. Limnología \\
\hline \multirow{4}{*}{$\begin{array}{l}\text { Región IV de } \\
\text { Coquimbo } \\
\text { Región V de } \\
\text { Aconcagua y } \\
\text { Región } \\
\text { Metropolitana } \\
\text { Región VI del } \\
\text { Gral. O'higgins }\end{array}$} & Río Choapa & 1 & $1982-1984$ & Comte et al. \\
\hline & Río Aconcagua & 9 & $2001-2006$ & CADE IDEPE, EDIC. \\
\hline & $\begin{array}{l}\text { Costeras } \\
\text { (Maipo) }\end{array}$ & 4 & 2007 & Fernández \\
\hline & Río Rapel & 21 & $2002-2006$ & $\begin{array}{l}\text { M\&P Ltda, Conc. Convento } \\
\text { Viejo, Geotécnica. }\end{array}$ \\
\hline \multirow{2}{*}{$\begin{array}{l}\text { Región VII del } \\
\text { Maule }\end{array}$} & Río Mataquito & 12 & $2000-2006$ & DGA, Est. y Gest. Ambiental \\
\hline & Río Maule & 21 & $2000-2006$ & $\begin{array}{l}\text { A\&C Cons. Gesam Cons., } \\
\text { Ingendesa, }\end{array}$ \\
\hline \multirow{3}{*}{$\begin{array}{l}\text { Región VIII de } \\
\text { Biobío }\end{array}$} & Río Itata & 10 & $1993-2000$ & Habit, DGA \\
\hline & Río Biobío & 9 & $2001-2003$ & Habit \\
\hline & Río Andalién & 17 & $1986-2000$ & Habit, Ruiz \\
\hline \multirow{2}{*}{$\begin{array}{l}\text { Región IX de } \\
\text { la Araucanía }\end{array}$} & Río Imperial & 22 & $1997-2003$ & Campos, DGA, Gutiérrez \\
\hline & Río Toltén & 5 & 1997 & Campos \\
\hline \multirow{2}{*}{$\begin{array}{l}\text { Región X de } \\
\text { los Lagos }\end{array}$} & Río Valdivia & 7 & $2005-2006$ & EULA \\
\hline & Río Bueno & 9 & $2003-2005$ & Velasco Asoc. \\
\hline
\end{tabular}

La utilización conjunta de los tres niveles de información (I, II y III) en la plataforma REC-Chile, permite visualizar por completo el rango de distribución de la especie en estudio. 

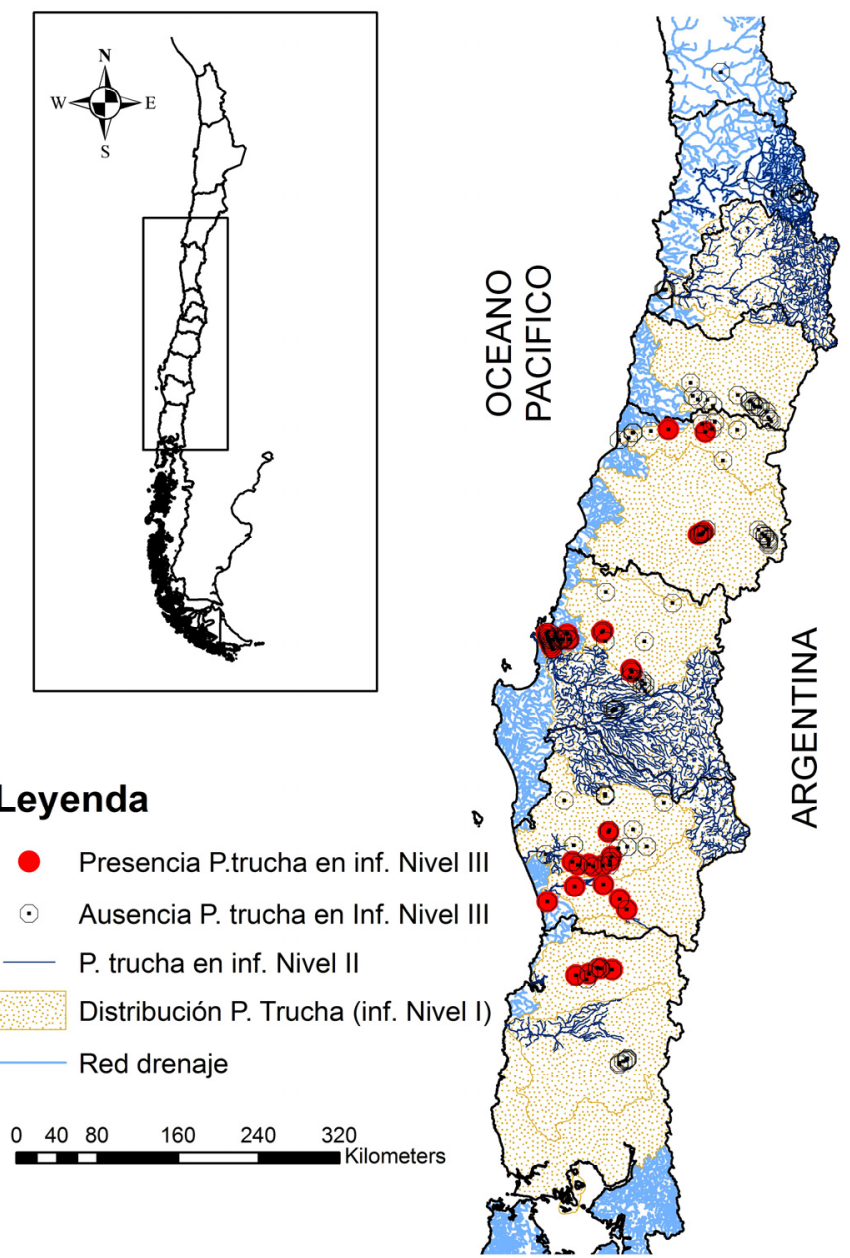

\section{Leyenda}

Figura 4.1. Representación gráfica de los
Niveles de información I, II y III, en el caso
ejemplo de la especie Percichthys trucha.

Poniendo como ejemplo la especie Percichthys trucha (Figura 4.1), se aprecia que el rango de distribución mostrado por la información Nivel I va desde la cuenca del río Aconcagua hasta el río Maullín (Dyer 2000a). La información Nivel II para esta especie se sitúa dentro del rango definido por Dyer (2000a), abarcando la totalidad de las cuencas Aconcagua, Maipo y Biobío, sin embargo, las cuencas intermedias no muestran información de Nivel II, y sólo en las cuencas Imperial, Toltén, Valdivia y Bueno se aprecia 
CAPÍTULO 4 BASE DE DATOS ECO-HIDROLÓGICA. que existe información sobre la presencia de esta especie en algunas de sus subsubcuencas. Por último, la información de Nivel III se distribuye entre los ríos Mataquito y Valdivia. Se observa también la ausencia de esta especie desde la cuenca del río Rapel hacia el norte. 


\subsection{DISCUSIÓN}

La información del Nivel I es la más abundante en extensión geográfica, pero también es aquella con mayor incertidumbre, puesto que para una misma especie diversos autores difieren en el rango de distribución, por ejemplo, para Nematogenys inermis, Campos et al. (1993) señalan que está distribuida entre los ríos Rapel y Biobío, pero Dyer (2000a) señala que la distribución corresponde entre los ríos Maipo y Biobío. Otro ejemplo sucede con Percilia gillissi a la cual Vila et al. (1999) la distribuye entre los ríos Maipo a Maullín, pero Dyer (2000a) extiende esa distribución por el norte hasta la cuenca del Aconcagua. A esta incertidumbre de la distribución de la especie se le debe agregar el hecho que bajo este nivel de información no se puede deducir sectores específicos de presencia o distribución de una especie dentro de una cuenca, por tanto, es imposible indicar si la especie se ubica preferentemente en zona de desembocadura o de cabecera, etc., dentro de una cuenca. En consecuencia, se asume que la especie se distribuye en toda cuenca dentro del rango geográfico definido para ésta.

La información íctica del Nivel II trabaja a una escala menor que el Nivel I, por lo cual la precisión en la ubicación, distribución y preferencias de hábitat de la especie es mayor, pudiendo así incorporar 450 registros para este nivel. La escala espacial mínima de distribución viene dada por la subsubcuenca, por tanto, se supondrá que la especie se distribuye en toda la subsubcuenca (como mínimo) a pesar de que el autor la haya señalado sólo para una localidad determinada. Dado el tipo de información perteneciente a este nivel, se puede deducir la presencia de una especie, pero no su ausencia, 
CAPÍTULO 4 BASE DE DATOS ECO-HIDROLÓGICA. puesto que sólo se indica la subsubcuenca, subcuenca y/o cuenca en la cual se muestreó la especie, pero no en las que la especie está ausente.

La información del Nivel III define el nivel más completo de la base de datos, contiene 171 estaciones de muestreo con 1010 registros. Como en cada estación de muestreo se indica la totalidad de las especies obtenidas en las campañas de muestreo, este nivel no sólo permite determinar presencia de una especie sino también la ausencia de ésta. El rango geográfico de este nivel es el más limitado de los tres niveles, definiéndose entre las cuencas del río Lluta y la cuenca del río Bueno. Hacia el sur de éste no hay datos.

En la zona Norte de Chile $\left(18^{\circ}-26^{\circ} \mathrm{S}\right)$, la información es escasa y se traduce en algunos puntos tomados en sectores altiplánicos y a lo largo de río Loa. En la zona denominada el Norte Chico $\left(26^{\circ}-31^{\circ} \mathrm{S}\right)$, los puntos de muestreos son heterogéneamente distribuidos, los ríos Huasco y Copiapó cuentan con información reciente, sin embargo, los muestreos en el río Choapa datan de 1987. Más preocupante son los casos de los ríos Elqui y Limarí que no presentan información de Nivel III.

La zona Central de Chile $\left(31^{\circ}-39^{\circ} \mathrm{S}\right)$ tiene una mayor densidad de información por cuenca, sin embargo, existen cuencas que no presentan puntos de muestreo, como es el caso de los ríos Maipo, Mapocho y Cachapoal.

En la zona Sur $\left(39^{\circ}-42^{\circ} \mathrm{S}\right)$, la densidad disminuye drásticamente, distinguiéndose dos zonas. La primera de ellas, comprendida entre los ríos Imperial y Bueno, presenta una buena cantidad de información, a excepción 
CAPITULO 4 BASE dE DATOS ECO-HIDROLÓGICA.

del río Toltén que presentan una baja densidad de puntos y no presenta información actualizada. La segunda zona se ubica desde la cuenca del Río Maullín hacia el sur, en donde la cantidad de información de Nivel III es nula.

Es importante destacar que existen cuencas en las cuales no existe información científica disponible de Nivel III, como por ejemplo, en las cuencas del río Aconcagua, Maipo, Rapel, Maule, etc. (Tabla 4.2). La información disponible en estas cuencas es información procedente de proyectos de ingeniería que según la Ley 19.300 deben someterse al Sistema de Evaluación de Impacto Ambiental.

La cantidad de registros de información en los niveles II y III para cada especie va acorde al estado de conservación de las especies definido en Habit et al. (2006). Las especies consideradas como "Vulnerable" presentan una mayor cantidad de registros y aquellas con un alto endemismo y/o consideradas como en "Peligro de extinción" o "Indeterminadas" tienen escasa cantidad de registros. El género Orestias es un claro ejemplo, ya que su marcado endemismo va acorde a una baja cantidad de registros. De la misma forma, las especies distribuidas en el norte de Chile (B. semotilus, $T$. chungaraensis y $T$. laucaensis) y las distribuidas en el extremo sur $(H$. macrei y $G$. globiceps) también presentan baja cantidad de registros. Un caso especial lo presenta la especie $O$. brevianalis que está considerada como vulnerable y el rango de distribución comprende las cuencas Elqui y Chiloé (Dyer 2000a), sin embargo, sólo presenta registros en la información del Nivel II. Esto quiere decir que al no presentar registros en la información de Nivel III ningún autor ha encontrado esta especie en los muestreos 
CAPÍTULO 4 BASE DE DATOS ECO-HIDROLÓGICA. ubicados dentro del rango de distribución. Por tanto, podría sugerirse reconsiderar el estado de conservación de esta especie.

La utilización de los tres niveles de información permite aprovechar la escasa información dulceacuícola existente, pudiendo utilizarse como complementarios o en su caso para redelimitar la distribución de una especie. Para el caso ilustrado en la figura 4.1, se indica que el rango de distribución de la especie $P$. trucha definida por los niveles I y II está comprendido entre los ríos Aconcagua y Maullín; sin embargo la información de Nivel III señala que se distribuye entre los ríos Mataquito y Valdivia, presentando una ausencia hacia el norte del río Rapel, lo cual contradice al rango de distribución definido en la literatura (Dyer 2000a, Vila et al. 1999, Campos et al. 1993). Si se considera que los muestreos en las cuencas Aconcagua y Maipo son recientes, se podría deducir que el rango de distribución para esta especie está en retroceso.

La inclusión de información hidrológica y fisicoquímica a la base de datos expande las posibilidades de uso de esta base de datos, permitiendo abordar los problemas y sus soluciones de una manera más holística. Además, al incorporar las estaciones fluviométricas y fisicoquímicas que están actualmente suspendidas, permitiría conocer el estado anterior de la calidad de las aguas en los ríos y eventualmente estos niveles podrían ser usados como niveles de referencia en posteriores estudios. 


\subsection{CONCLUSIÓN}

Se entrega, ante la comunidad científica, una base de datos ecohidrológica confeccionada a partir de la recopilación de información hidrológica, fisicoquímica e íctica que contiene información de diversa escala espacial. Esta base de datos se implementó en un Sistema de Información Geográfico, con sistemas de coordenadas PSAD 1956 UTM Huso 18S.

Esta base de datos está acoplada a la plataforma de gestión "Clasificación Eco-Hidrológica de los ríos de Chile" (REC-Chile), transformándose así, en una herramienta eficaz para sentar las bases de proyectos de gestión, planificación y conservación del medio acuático. 


\subsection{BIBLIOGRAFÍA}

ARRATIA G. 1983. Preferencias de hábitat de peces siluriformes de aguas continentales de Chile (Fam. Diplomystidae y Trichomycteridae). Studies on Neotropical Fauna and Environment 18 (4): 217-237.

BAHAMONDES I, D SOTO \& I VILA. 1979. Hábitos alimentarios de los pejerreyes (Pisce:Atherinidae) del Embalse Rapel. Medio Ambiente 4 (1): 3-18.

BENETTI AD, AE LANNA \& MS COLBACHINI. 2004. Current practices for establishing environmental flows in Brazil. River Research and Applications 20: 427-444.

BOND NR \& PS LAKE. 2003. Characterizing fish-habitat associations in streams as the first step in ecological restoration. Austral Ecology 28: 611-621.

CAMPOS H, V RUIZ \& JF GAVILÁN. 1993. Peces del río Biobío. Publicaciones de divulgación. EULA 5. 100pp.

COMTE S \& I VILA. 1987. Modalidad reproductiva de Basililchthys microlepidotus (Jenyns), en el río Choapa (Pisces:Atherinidae). Anales Museo de Historia Natural, Valparaíso 18: 85-94.

CUSSAC V, S ORTUBAY, G IGLESIAS, D MILANO, M LATTUCA, J BARRIGA, M BATTINI \& M GROSS. 2004. The distribution of South American galaxiid fishes: the role of biological traits and post-glacial history. Journal of Biogeography 31: 103-121.

DYER B. 2000a. Revisión sistemática y biogeográfica de los peces dulceacuícolas de Chile. Estudios Oceanológicos, Antofagasta 19: 77-98.

DYER B. 2000b. Revisión sistemática de los pejerreyes de Chile (TELEOSTEI, ATHERINIFORMES). Estudios Oceanológicos, Antofagasta 19: 99-127.

HABIT E. 1998. Análisis de la dieta de Percilia gillissi (Pisces: Percilidae) en poblaciones del río y canales de riego (Cuenca del Itata, VIII Región) Theoria 7: $33-46$.

HABIT E. 2005. Aspectos de la biología y hábitat de un pez endémico de Chile en peligro de extinción (Diplomystes nahuelbutaensis Arratia, 1987). Interciencia 30 (1): 8-11. 
CAPITULO 4 BASE dE DATOS ECO-HIDROLÓGICA.

HABIT E \& M BELK. 2007. Threatened fishes of the world: Percilia irwini (Eigenmann 1927) (Perciliidae). Environmental Biology of Fishes 78: 213-214.

HABIT E, P VICTORIANO \& H CAMPOS. 2003. Ecología trófica y aspectos reproductivos de Trichomycterus areolatus (Pisces, Trichomycteridae) en ambientes lóticos artificiales. Revista de Biología Tropical 53 (1-2): 195-210.

HABIT E, B DYER \& I VILA. 2006. Estado de conocimiento de los peces dulceacuícolas de Chile. Gayana 70 (1): 100-112.

MURILLO V \& VH RUIZ. 2002. El puye Galaxias globiceps EIGENMANN 1927 (Osteichthyes: Galaxiidae): ¿Una especie en peligro de extinción?. Gayana 66 (2): 191-197.

NOBLE R, R COWX, D GOFFAUX \& P KESTEMONT. 2007. Assessing the health of European rivers using functional ecological guilds of fish communities: standardising species classification and approaches to metric selection. Fisheries Management and Ecology 14: 381-392.

PARDO R. 2002. Diferenciación morfológica de poblaciones de Trichomycterus areolatus Valenciennes 1846 (Pisces: Siluriformes: Trichomycteridae) de Chile. Gayana 66 (2): 203-205.

PARDO R, S SCOTT \& I VILA. 2005. Análisis de formas en especies chilenas del género Trichomycterus (Osteichthyes: Siluriformes) utilizando morfometría geométrica. Gayana 69 (1): 180-183.

PEREDO S \& C SOBARZO. 1994. Actividad gonádica estacional de Galaxias maculatus (Jenyns, 1842) en el río Cautín IX Región, Chile. Boletín Sociedad Biología, Concepción 65: 65-70.

PEREDO-PARADA M, F MARTÍNEZ-CAPEL, JD ALCARAZ \& M BARGAY. 2009. Potential geographical distribution fo two endemic freshwater fishes $(P$. gillissi and D. camposensis) of Chile. Proceedings of $7^{\text {th }}$ international Symposium on Ecohdraulics. Concepcion. Chile. ISBN: 978-978-08-2100-5.

PEREDO-PARADA $\mathrm{M}, \mathrm{F}$ MARTÍNEZ-CAPEL, D QUEVEDO \& A HERNÁNDEZ-MASCARELL. 2010. Implementation of an Eco-Hydrological Classification in Chilean rivers. Gayana. En prensa.

RUIZ VH, R FIGUEROA \& B NEIRA 2006. Índice bibliográfico sobre peces de aguas continentales y estuarinas de Chile. Boletín Sociedad Biología de Concepción 77: 73-110. 
CAPÍTULO 4 BASE DE DATOS ECO-HIDROLÓGICA. SCHUMTZ S, I COWX, G HAIDVOGL \& D PONT. 2007. Fish-based methods for assessing European running waters: a synthesis. Fisheries Management and Ecology 14: 369-380.

SCOTT S, R PARDO \& I VILA. 2007. Trophic niche overlap between two Chilean endemic species of Trichomycterus (Teleostei: Siluriformes). Revista Chilena de Historia Natural 80 (4): 431-437

SNELDER T \& B BIGGS. 2002. Multiscale river environment classification for water resources management. Journal of the American Water Resources Association 38 (5): 1225-1239.

SNELDER T \& KFD HUGHEY. 2005. The use of an Ecological Classification to improve Water Resource Planning in New Zealand. Environmental Management 36 (5): 741-756.

SNELDER T, B BIGGS \& M WEATHERHEAD. 2004a. Nutrient concentration criteria and characterization of patterns in trophic state for rivers in heterogeneous landscapes. Journal of the American Water Resources Association 40 (1): 1-14.

SNELDER T, F CATTANÉO, A SUREN \& B BIGGS. 2004b. Is the River Environment Classification an improved landscape-scale classification of rivers?. Journal of North American Benthological Society 23 (3): 580-598.

SNELDER T, B BIGGS \& R WOODS. 2005. Improved eco-hidrological classifications of river. River Research and Applications 21: 609-628.

SOTO D, I ARISMENDI, J GONZALEZ, J SANZANA, F JARA, C JARA, E GUZMAN \& A LARA. 2006. Southern Chile, trout and salmon country: invasion patterns and threats for native species. Revista Chilena de Historia Natural 79 (1): 97-117.

THARME RE. 2003. A gobal perspective on environmental flow assessment: emerging trends in the development and application of environmental flow methodologies for rivers. River Research and Applications 19: 397-441.

VILA I. 2001. La diversidad acuática: estructura, funciones y salud de las cuencas, bases para el Manejo Integrado en Sustentabilidad, Proyecto PMRH, Ministerio de Obras Públicas, Gobierno de Chile.

VILA I. 2006. A new species of killifish en the genus Orestias (Teleostei: Cyprinodontidae) from the Southern high Andes, Chile. Copeia 3: 472-477.

VILA I, L FUENTES \& M CONTRERAS. 1999. Peces límnicos de Chile. Boletín Museo de Historia Natural, Chile 48: 61-75. 
CAPITULO 4 BASE dE DATOS ECO-HIDROLÓGICA.

VILA I, R PARDO, B DYER \& E HABIT. 2006a. Peces límnicos: Diversidad, origen y estado de conservación. En: Vila I, A Veloso, R Schlater \& C Ramirez (eds.) Macrófitas y vertebrados de los sistemas límnicos de Chile: 73-103. Editorial Universitaria, Stgo, Chile.

VILA I, M MENDEZ, S SCOTT, P MORALES \& E POULIN. 2006b. Threatened fishes of the world: Orestias ascotanensis Parenti, 1984 (Cyprinodontidae). Environmental Biology Fisheries 80: 491-492. 


\subsection{ANEXOS}

\subsubsection{Literatura citada en la base datos}

\subsubsection{Información Nivel I}

DYER B (2000a) Revisión sistemática y biogeográfica de los peces dulceacuícolas de Chile. Estudios oceanológicos. 19:77-98 ISSN 0071-173X.

DYER B (2000b) Revisión sistemática de los pejerreyes de Chile (TELEOSTEI, ATHERINIFORMES). Estudios oceanológicos 19:99-127

CAMPOS H, RUIZ V \& JFGAVILÁN (1993) Peces del río Biobío. Publicaciones de divulgación. Eula 5 100pp.

HABIT E \& M BELK (2007) Threatened fishes of the world: Percilia irwini (Eigenmann 1927) (Preciliidae). Environmental Biology 78:213-214

VILA I, FUENTES L \& M CONTRERAS (1999). Peces límnicos de Chile. Boletín. Museo Historia Natural, Chile 48:61-75

\subsubsection{Información Nivel II}

ARRATIA G (1983) Preferencias de hábitat de peces siluriformes de aguas continentales de Chile (Fam. Diplomystidae y Trichomycteridae). Studies on Neotropical Fauna and Environment 18 (4): 217-237.

CAMPOS H (1984) Productividad íctica de ríos y lagos araucanos. Taller internacional sobre ecología y manejo de peces en lagos y embalses.FAO.Stgo, Chile. Vila y Fagetti (eds). 4:237 pp.

CAMPOS H (1985). Distribution of the fishes in the andean rivers in the South of Chile. Arch. Hydrobiol. Suppl. 104 (2): 169-191.

CAMPOS H, RUIZ V \& JFGAVILÁN (1993) Peces del río Biobío. Publicaciones de divulgación. Eula 5 100pp. 
CAPITULO 4 BASE de DATOS ECO-HIDROLÓGICA.

HABIT E, VICTORIANO P \& H CAMPOS (2003) Ecología trófica y aspectos reproductivos de Trichomycterus areolatus (pisces, Trichomycteridae) en ambientes lóticos artificiales. Rev. Biol. Trop. 53 (1-2): 195-210

HABIT E (2005a) Aspectos de la biología y hábitat de un pez endémico de Chile en peligro de extinción (Diplomystes nahuelbutaensis ARRATIA, 1987). Interciencia $30(1): 8-11$

HABIT E, BELK M, TUCKFIELD R \& O PARRA (2005b) Response of the fish community to human-induced changes in the Biobío River in Chile. Freshwater biology. Doi:10.1111/j.1365-2427.2005.01461.x

LÓPEZ M, VARGAS A \& G LOBOS (1999). Tramas tróficas y su importancia en estudios hidrológicos integrales, aplicación al río Polcura. VI jornadas del Conaphi-Chile.

MANRÍQUEZ A, HUAQUIN L \& M ARELLANO (1984) Peces nativos y problemática planterada para la inducción de desove. Taller internacional sobre ecología y manejo de peces en lagos y embalses.FAO.Stgo, Chile. Vila y Fagetti (eds). 4:237 pp.

OLMOS VL, VICTORIANO P, HABIT E \& C VALDOVINOS (2003) Parásitos de peces nativos de la cuenca del río Laja (Chile Central) y alcances sobre sus ciclos de vida. Arch. Med. Vet. 35 (2): 195-203.

PEREDO S \& C SOBARZO (1994) Actividad gonádica estacional de Galaxias maculatus (JENYNS, 1842) en el río Cautín IX Región, Chile. Boletin Sociedad Biologica, Concepción. 65:65-70

QUANTITATIVA LTDA (2004) Antecedentes respecto a la biodiversidad acuática en la cuenca del Maipo. Informe técnico. 98 pp.

SIGA INGENIERÍA \& CONSULTORÍA (2003) Estudio de Impacto Ambiental "Embalse Ancoa, sitio Ancoa alto, VII Región" Informe final. Dirección General de Obras Hidráulicas. Ministerio de Obras Públicas. Gobierno de Chile

UNIVERSIDAD DE CHILE (1998) Análisis de criterios hidroambientales en el manejo de recursos hídricos. Pautas para la determinación de caudales ecológicos. Informe técnico. Dirección General de Aguas. 199 pp.

VARGAS C, CONTRERAS M \& I VILA (2002). Edad y crecimiento de Odontesthes brevianalis (GÜNTHER 1880) en la laguna Conchalí $\left(31^{\circ} 35^{\prime} ; 71^{\circ} 32^{\prime}\right)$, Chile. Gayana. 66 (2): 199-202 
CAPÍTULO 4 BASE DE DATOS ECO-HIDROLÓGICA. VILA I, FUENTES L \& M CONTRERAS (1999). Peces límnicos de Chile. Boletín Museo Historia Natural, Chile. 48:61-75.

VILA I (2006). A new species of killifish in the Genus Orestias (Telestei:Cyprinodontidae) form high Andes, Chile. Copeia 2006 (3):472-477

\subsubsection{Información Nivel III}

AYALA Y CABRERA CONSULTORES (2001) Estudio de Impacto Ambiental Embalse Ancoa, VII región. Informe final. Dirección Regional de Obras Públicas. Ministerio de Obras Públicas. Gobierno de Chile.

CADE IDEPE 2006. Estudio de Impacto Ambiental Proyecto Embalse Puntilla del Viento. Ministerio de Obras Públicas. Gobierno de Chile.

CAMPOS H (1998) Manejo e investigación aplicada polación de salmonídeos de importancia deportiva, IX región. Informe técnico para SERNAPESCA. Universidad Austral de Chile.

COMTE S \& I VILA (1987) Modalidad reproductiva de Basilichthys microlepidotus (JENYNS), en el río Choapa. (PISCES: ATHERINIDAE). Anales Museo Historia Natural $18: 85-94$.

CONCESIONARIA CONVENTO VIEJO (2006) Declaración de Impacto Ambiental Canal Matriz Nilahue, Tramo II. Obras de Regadío Embalse Convento Viejo Etapa II. Dirección de Obras Hidráulicas. Ministerio de Obras Públicas. Gobierno de Chile.

DIRECCIÓN GENERAL DE AGUAS (2002) Programa de manejo de recursos hídricos a nivel de cuencas hidrográficas. Ministerio de Obras Públicas, Unidad Técnica PMRH. Santiago.

EDIC INGENIEROS CONSULTORES (2003) Estudio de Impacto Ambiental Central Hidroeléctrica Hornitos. Informe Final. Hidroeléctrica Guardia Vieja. S.A.

EULA (2007) Estudio de Impacto Ambiental Central Hidroeléctrica San Pedro. Colbun S.A.

ESTUDIO Y GESTIÓN AMBIENTAL SA (2006). Linea base ambiental. Planta Licancel. Celulosa Arauco y Constitución S.A. Informe final. 275 pp

FERNÁNDEZ MAC-AULIFFE. 2007. Estudio de Impacto Ambiental Embalse Tricao. Region V. 
CAPITULO 4 BASE dE DATOS ECO-HIDROLÓGICA.

GEOTÉCNICA CONSULTORES (2003) Estudio de Impacto Ambiental Embalse Convento Viejo. Informe final. Dirección de Obras Hidráulicas. Ministerio de Obras Públicas. Gobierno de Chile.

GESAM CONSULTORES LTDA (2002) Estudio específico de línea de base flora y fauna acuática y calidad de las aguas: Proyecto Embalse Ancoa, VII Región. Informe Final Ministerio de Obras Públicas. Gobierno de Chile

GUTIERREZ J (2005) Estudio del ensamble de peces de la subcuenca del río Traiguén,IX región, y su relación con la calidad del agua. Tesis de Licenciatura, Facultad de Ciencias, Universidad Católica de Temuco. 114 pp.

HABIT E (1994). Contribución al conocimiento de la fauna íctica del río Itata. Boletín Sociedad Biológica 65: 143-147.

HABIT E, VICTORIANO P \& O PARRA (2002) Translocación de peces nativos en la cuenca del río Laja (región del Biobío, Chile). Gayana 66 (2):181-190

HABIT E, VICTORIANO P \& A RODRÍGUEZ-RUÍZ (2003) Variaciones espacio-temporales del ensamble de peces de un sistema fluvial de bajo orden del centro-sur de Chile. Revista Chilena de Historia Natural 76: 3-14

HABIT E, PARRA O \& C VALDOVINOS (2005) Ictiofauna de un sistema fluvial receptor de aguas servidas: respuestas a una nueva planta de tratamiento (río Quilque, Chile central). Gayana 69 (1):94-103

INGENDESA SA (2007) Estudio de Impacto Ambiental Proyecto Central Hidroeléctrica Los Cóndores. Informe Final. Endesa Chile. Informe Final.

JARA F, SOTO D \& R PALMA (1995) Reproduction in Captivity of the Endangered Killifish Orestias ascotanensis (Teleostei: Cyprinodontidae). Copeia, 1995 (1):226-228

RUIZ VH (1993) Icthyofaune of the Andalien Basin (Concepción, Chile). Gayana $1: 109$.

UNIVERSIDAD DE CHILE (2006) Obtención de la información para la clasificación de la fauna acuática continental de la I a la III región. Informe. Servicio Nacional de Pesca. Gobierno de Chile.

VELASCO V ASOC (2003) Estudio de Impacto Ambiental "Proyecto Hidroeléctrico Licán. X Región”. Informe final. Inversiones Candelaria Ltda.

VELASCO V ASOC (2005) Declaración Impacto Ambiental "Modificación Proyecto Hidroeléctrico Licán". Candelaria Inversiones Ltda. 
Capitulas

\section{Determinación de la distribución}

geográfica potencial de las especies

dulceacuícolas 



\subsection{RESUMEN}

Entre la falta de información sobre los ecosistemas acuáticos en Chile, destaca la falta en el conocimiento de la distribución geográfica de las especies ya que éste es uno de los aspectos más relevantes para definir planes de conservación. Los Modelos de Distribución Espacial (MDE) se han convertido en una herramienta muy utilizada por los ecólogos para determinar los rangos de distribución geográfica de las especies. Para suplir la falta de información en Chile, se ha planteado utilizar las clases de la clasificación Eco-Hidrológica de los ríos de Chile (REC-Chile) como variables de entrada en modelos de distribución espacial.

Se utilizó la técnica Random Forests (RF) para desarrollar 32 MDE, uno para cada especie íctica nativa analizada. Se evaluó el poder de discriminación de los 6 niveles de la REC-Chile sobre la distribución espacial de las especies dulceacuícolas nativas de Chile mediante el análisis de disimilaridades, obteniéndose resultados satisfactorios $(\mathrm{Cs}=0.248)$. De las 32 modelos RF analizados, 18 entregaron resultados buenos resultados con AUC y Kappa mayores que 0.739 y 0.265 respectivamente. De las clases REC-Chile utilizadas, las categorías del factor Clima y las del factor Origen de Flujo son las variables que más influyen sobre la distribución espacial de las especies.

Pareciera que la utilización de la REC-Chile, permite suplir la falta de información para determinar MDE necesarios para determinar planes de conservación. 


\subsection{INTRODUCCIÓN}

El aislamiento biogeográfico y las características hidrológicas de los ríos de

Chile son los principales factores que justifican el alto endemismo, la baja riqueza de especies dulceacuícolas y la tendencia generalizada a poseer tamaños pequeños (Habit et al. 2006, Dyer 2000, Vila et al. 1999). Lo anterior se traduce en un alto valor biogeográfico y ecológico de estas comunidades. Sin embargo, dichas especies presentan grandes problemas de conservación, considerándose cerca del $40 \%$ en peligro de extinción (Habit et al. 2007). La principal causa de este estado es el deterioro de su hábitat por razones antrópicas (Orrego et al. 2009, Habit et al. 2006, Dyer 2000, Vila et al. 1999) así como posibles desplazamientos por competencia por con especies introducidas (Pardo et al. 2009, Habit et al. 2006, Soto et al. 2006).

Dadas estas condiciones, se hace necesario implementar planes de gestión integral de cuencas que garanticen la conservación de la biodiversidad íctica, lo que implica tener un conocimiento detallado de las especies y su distribución geográfica (Elith et al. 2006). Así pues, los modelos de distribución de especies (MDE) se han transformado en una herramienta muy útil para desarrollar planes de conservación (Hopkins II \& Burr 2009). Sin embargo, la calidad y cantidad de información de las especies nativas en Chile es deficiente, por ser escasa, fragmentada y poco actualizada (PeredoParada et al. 2009), resultando necesario ahondar en la distribución, abundancia, sistemática y biología de las especies (Habit et al. 2006), así 
CAPITULO 5 DISTRIBUCIÓN GEOGRÁFICA POTENCIAL. como en sus patrones de distribución y biodiversidad (Oyanedel et al. 2008).

Los MDE han tenido una alta aceptación en el área de la eco-informática. Ésta es un área interdisciplinaria que promueve el uso de tecnología computacional avanzada para revelar procesos ecológicos y patrones a través de los niveles de complejidad del ecosistema (Recknagel 2003). Estos MDE son capaces de modelar las relaciones no lineales y complejas que existen en la ecología (Olden et al. 2008) con un alto poder de explicación y predicción de los patrones ecológicos.

El uso de este tipo de técnicas en la ecología acuática ha ido en aumento a medida que los resultados han demostrado una gran eficiencia en su implementación, así como, la posibilidad de generar mapas de predicción mediante los Sistemas de Información Geográfica (SIG). Por lo general, los MDE presentan variables ambientales, de diversa índole y escala, como variables predictoras, y los datos utilizados en el entrenamiento de estas técnicas provienen de observaciones de presencia/ausencia.

Entre las técnicas más utilizadas en estudios de ecosistemas acuáticos se encuentran Artificial Neuronal Networks (Peter et al. 2007, Oakes et al. 2005, Aguilar et al. 2003), Classification and Regression Trees (Sowa et al. 2007, Oakes et al. 2005), Boosted Regression Trees (Hopkins II \& Burr 2009), Assessment by a Nearest Neighbour Analysis (Hermoso et al. 2009, Mugodo et al. 2006), Genetics Algorithms for Rule Set Prediction (Domínguez-Domínguez et al. 2006, McNyset 2005), Generalized Aditive 
CAPÍTULO 5 DISTRIBUCIÓN GEOGRÁFICA POTENCIAL.

Models (Buisson et al. 2008, Leathwick et al. 2006), Multivariate Adaptive Regression Splines (Leathwick et al. 2006), entre otros.

Algunos estudios han comparado diversas técnicas (Tirelli \& Pessani 2009, Marmion et al. 2008, Leathwick et al. 2006, Olden et al. 2002). Otros han analizado su comportamiento bajo distintas escalas espaciales de trabajo (Hopkins II \& Burr 2009, Olden et al. 2006, Brosse et al. 2003).

Las características de la información existente en Chile (al menos en algunas cuencas hidrográficas) no permiten implementar MDE con resultados confiables y con poder de discriminación, ya que para esto se requeriría un modelo de datos adecuado a la escala espacial y al objetivo de implementación (Austin 2007), evitando el sesgo y/o limitación de los resultados. Para suplir esta falta de información, las clasificaciones hidrológicas pueden tener un rol importante permitiendo deducir, ya sea de forma explícita o implícita, información de variables ambientales de cada una de sus clases. Esta información podría ser utilizada como variables predictoras en los MDE.

La Clasificación Eco-Hidrológica de los ríos de Chile (REC-Chile) desarrollada por Peredo-Parada et al. (2010) es del tipo a priori (sensu Snelder et al. 2005) basada en que el clima, la topografía y la geología son los principales causantes de la variación espacial del patrón hidrológico en Chile. Como resultado, la clasificación REC-Chile se construyó a partir de la superposición jerárquica de seis factores controladores (Clima, Origen de flujo, Geología, Posición relativa de subsubcuenca, Uso del suelo y Pendiente del tramo) según su escala espacial de actuación. 
Esta estructura jerárquica es flexible y permite definir niveles de clasificación. Dichos niveles se definen de acuerdo a cuales son los factores controladores que se han superpuesto. El primer nivel corresponde al primer factor controlador (Clima); el segundo nivel corresponde a la superposición jerárquica de los dos primeros factores (Clima y Origen de Flujo); el tercer nivel se define agregando jerárquicamente el tercer factor (Geología) al segundo nivel de clasificación. De la misma forma se obtienen los sucesivos niveles jerárquicos de clasificación hasta el sexto y último nivel definido como la superposición jerárquica de todos los factores controladores.

Cada factor está dividido en categorías de carácter cualitativo. Las clases REC-Chile se construyen a partir de dichas categorías y de acuerdo al nivel de clasificación. Como resultado se obtiene una clasificación con estructura de árbol donde las categorías del factor i es dividido entre las categorías del factor $\mathrm{i}+1$ conformando las clases del nivel $\mathrm{i}+1$.

Las características de la REC-Chile permiten utilizar sus clases como variables predictoras en diversas técnicas MDE. Éstas son su estructura jerárquica, flexibilidad para situarse bajo distintas escalas espaciales, posibilidad de clasificar los tramos de ríos bajos distintos objetivos de clasificación, su unidad de clasificación es el tramo de río, así como su facilidad para la interpretación de las clases, tanto hidrológicas como de calidad geoquímica de las aguas (Ver Capítulo 3).

Este tipo de estructura jerárquica de la REC-Chile permite abarcar diversas escalas espaciales en la obtención de los predictores lo que resulta coherente 
CAPÍTULO 5 DISTRIBUCIÓN GEOGRÁFICA POTENCIAL.

con la teoría de la estructura jerárquica de los ecosistemas (Frissell et al. 1986) y a su vez, permitiría obtener mejor precisión en los MDE (Olden et al. 2006), así como en una correcta interpretación ecológica de los MDE.

La técnica Random Forests (RF) desarrollada por Breiman (2001) es una técnica de MDE que no ha sido muy difundida en la limnología. Ésta ha sido utilizada principalmente para determinar distribución espacial de especies vegetales (Leng et al. 2008, Cutler et al. 2007, Peters et al. 2007, Prasad et al. 2006). El funcionamiento de RF, en base a múltiples árboles de decisión no podados construidos a partir de una elección aleatoria de los datos, le atañen resultados sin sesgo y con una alto poder de discriminación eliminando el sobreentrenamiento (Cutler et al. 2007, Breiman 2001).

Sumado a lo anterior y considerando que RF permite incorporar variables categóricas como predictores y que es capaz de desarrollar un MDE con una escasa cantidad de datos (Breiman 2001), RF en una técnica de interés para aplicar utilizando la escasa información íctica en Chile y las clases RECChile como variables predictoras.

El objetivo de este capítulo es realizar modelos de distribución espacial de las especies nativas de Chile mediante la técnica Random Forests utilizando las clases REC-Chile como variables predictoras. El estudio responde dos interrogantes: 1) ¿La clasificación REC-Chile es una clasificación capaz de discriminar la distribución espacial de los peces nativos de Chile? 2) ¿Es posible desarrollar modelos predictivos mediante Random Forests utilizando las clases REC-Chile como variables predictoras? 


\subsection{METODOLOGÍA}

\subsubsection{Datos y variables}

Se han utilizado los datos correspondientes al Nivel III de la base de datos Eco-Hidrológica de los ríos de Chile (Peredo-Parada et al. 2009) considerando cada esfuerzo de pesca como un dato independiente. Los esfuerzos de pesca no están estandarizados y diversas técnicas fueron realizadas en los muestreos. Esto podría conllevar a una pseudoreplicación de datos con las consecuencias estadísticas que serán discutidas posteriormente.

Se consideraron 32 especies nativas con un total de 485 esfuerzos de pesca realizados entre los años 1987 y 2008 distribuidos por tramos de río de toda la clasificación REC-Chile, considerándose solo datos de presencia/ausencia. A cada esfuerzo de muestreo se le asignó el código REC-Chile del tramo de río en donde fue realizado. La interpretación de este código permitió asociar a cada esfuerzo de pesca las clases REC-Chile correspondientes, las cuales fueron utilizadas como variables independientes en el modelo de predicción de presencia/ausencia de peces nativos.

Con el fin de disminuir el número de clases REC-Chile en la construcción del modelo predictivo, y por tanto el número de variables, se agruparon las categorías Llano (L) y Valle (V) del factor controlador Origen de Flujo justificada por su similitud hidrológica (Ver Capítulo 3). Análogamente, se agruparon las categorías rocas Carbonatadas (Ca) y rocas Volcánicas 
CAPÍTULO 5 DISTRIBUCIÓN GEOGRÁFICA POTENCIAL.

fracturadas (Vf) por su similitud geoquímica (Ver Capítulo 3). El número total de variables resultante es el número de clases asociada a cada nivel jerárquico de la REC-Chile. Es decir, 5 clases para el primer nivel, 18 clases para el segundo nivel, 40 para el tercero, 61, 86 y 105 clases para los niveles cuarto, quinto y sexto, respectivamente.

\subsubsection{Evaluación de la clasificación REC-Chile}

Una completa evaluación de un sistema de clasificación a priori (sensu Snelder et al. 2005) consta de un test de robustez de la clasificación y su posterior evaluación con clasificaciones desarrolladas a partir de los mismos datos (clasificación a posteriori) utilizados en la evaluación de la clasificación a priori.

Un test de clasificación consiste en estimar la robustez de la clasificación mediante la comparación entre la heterogeneidad dentro de las clases y la homogeneidad entre las clases. Van Sickle (1997) propone comparar la similaridad media intra-clases $(\bar{W})$ con la similaridad media inter-clases $(\bar{B})$, es decir, da una idea de lo compactas y aisladas que están las clases entre sí.

Para esta clasificación se ha utilizado el estadístico Cs (Van Sickle \& Hughes 2000), definido como $\boldsymbol{C s}=\overline{\boldsymbol{B}}-\overline{\boldsymbol{W}}$. Al igual que el índice M (ver Capítulo 2), este índice permite cuantificar la robustez de una clasificación. En ecología de peces se utilizan preferentemente dos distancias para determinar la similaridad, o disimilaridad, entre dos estaciones de muestreo según el tipo de datos existente. Para datos de presencia o ausencia se utiliza 
CAPITULO 5 DISTRIBUCIÓN GEOGRÁFICA POTENCIAL. el índice de Jaccard (Legendre \& Legendre 1998), en caso de datos de biomasa se utiliza el índice de Bray-Curtis (Legendre \& Legendre 1998). Ambas distancias, debido a sus fórmulas, están acotadas entre 0 y 1 , por tanto, el índice Cs también lo está. Si no hay estructura de clase $\mathrm{Cs}=0$ ( $\overline{\boldsymbol{B}}=\overline{\boldsymbol{W}}$ ) y a medida que Cs aumenta, aumenta la estructura de clasificación (más distanciada están las clases con respecto a la distancia media entre ellas). En caso que $\mathrm{Cs}=1$ se consideraría que hay una clasificación perfecta. La significación estadística del test se determinó mediante un test de aleatoriedad $(\mathrm{n}=1000 ; \mathrm{p}<0.001)$ basado en la hipótesis nula de "No estructura de clases" utilizando la técnica de remuestreo de bootstrap sin reemplazo.

El estadístico Cs fue evaluado independientemente para los 6 niveles de la REC-Chile, es decir, considerando la acción superpuesta de los factores controladores pertenecientes a cada nivel. Se consideró un mínimo de 5 esfuerzos de pesca por cada clase de la REC-Chile. Se definió un $\Delta$ Cs para cuantificar la mejora en el nivel de ajuste de la clasificación REC-Chile al incorporar un nivel más (y por tanto, aumentar el número de clases)

El nivel de ajuste de la REC-Chile se comparó con 3 clasificaciones a posteriori: 1) Hierarchical clusters (HC) desarrollada mediante un análisis cluster jerárquico, 2) Distance cluster (DC) desarrollada mediante un análisis cluster de distancias y 3) Geographical regions (GR), desarrollada como una clasificación geográfica. Al generar estas tres clasificaciones $a$ posteriori se mantuvo la cantidad de niveles, así como el número de clases en cada nivel existente en la REC-Chile 
CAPÍTULO 5 DISTRIBUCIÓN GEOGRÁFICA POTENCIAL.

Los cálculos de las disimilaridades medias $\overline{\boldsymbol{W}}_{\boldsymbol{i}}, \overline{\boldsymbol{W}}, \overline{\boldsymbol{B}}$, el análisis de permutaciones aleatorias (bootstrap) y las clasificaciones a posteriori (HC, DC y GR) se realizaron mediante el paquete de funciones Env.Class desarrolladas por Snelder (comunic. Personal 2009) ambientadas en el lenguaje estadístico R versión 2.8.1 (R Development Core Team 2008)

\subsubsection{Modelo de predicción}

\subsubsection{Desarrollo del modelo}

Las clases REC-Chile asociadas a los esfuerzos de pesca fueron utilizadas como variables independientes para el desarrollo de un modelo predictivo de presencia/ausencia para 32 especies dulceacuícolas nativas. La cantidad de datos para cada especie se detalla en la Tabla 5.1. El modelo utilizado fue Random Forests (Breiman 2001). Este modelo es una nueva técnica avanzada del Machine Learning que genera múltiples árboles de decisión los cuales son promediados (mediante el sistema del árbol más "votado") para obtener un modelo final. Cada árbol es construido a partir de un subconjunto de datos de entrenamiento obtenidos mediante la técnica de remuestreo bootstrap con reemplazo al final de la construcción de cada árbol. Los nodos son divididos usando el mejor predictor entre un subconjunto $\mathrm{m}$ de variables predictoras elegidas aleatoriamente del total de variables (de aquí el nombre de Random). Se construyen entre 500 y 2000 árboles (de aquí el nombre de Forests).

Los árboles son desarrollados completamente hasta el final sin realizar ningún tipo de poda. Los datos que no fueron considerados en el entrenamiento del árbol son utilizados para calcular el out-of-bag error 160 
CAPITULO 5 DISTRIBUCIÓN GEOGRÁFICA POTENCIAL. (OOB), el cual se determina como el porcentaje de clasificaciones realizadas incorrectamente en este grupo de datos. El OOB es no sesgado, eliminando la necesidad de una validación cruzada o un conjunto de datos extra para validación (Breiman 2001). Este error se determina para cada árbol y posteriormente se promedia para todas las observaciones. Breiman (2001) mostró que RF posee valores acotados de errores generalizados, teniendo que ser el número de árboles generados suficientemente grande para que este error converja. Consecuentemente, en RF no es posible que exista sobreentrenamiento. La selección aleatoria de las variables predictoras disminuye la correlación entre los árboles no podados manteniendo un sesgo bajo.

La técnica RF tiene la capacidad para cuantificar la importancia de una variable predictora en el modelo. El procedimiento es el siguiente: Para cada árbol de decisión se determina el $\mathrm{OOB}$ y se cuenta el número de votos emitidos para cada clase correctamente clasificada (cuantas veces se predijo correctamente para cada clase). Al cambiar aleatoriamente el valor de una de las variables predictoras (variable j) se evalúa nuevamente el OOB y se vuelve a contabilizar el número de votos correctos para dichas clase. Se comparan ambas votaciones y promediando entre todos los árboles de decisión se obtiene el valor bruto de la importancia de la variable. Finalmente esta importancia se ajusta dependiendo de las correlaciones entre los árboles.

Esta técnica también posee otras ventajas. Es un modelo no paramétrico y no lineal. Permite trabajar tanto con variables numéricas y/o categóricas, sean éstas dicotómicas o no, y entrega valores medios de la importancia 
CAPÍTULO 5 DISTRIBUCIÓN GEOGRÁFICA POTENCIAL.

estimada de las variables individuales en la clasificación. Los casos sin clasificar son claramente identificados y los modelos obtenidos pueden ser fácilmente aplicados a nuevos datos. Adicionalmente, RF no tiene límites aparentes respecto de la complejidad de la base de datos, y por consiguiente, puede manipular bases de datos de gran magnitud que otros métodos del machine learning (Cutler et al. 2007, Breiman 2001)

\subsubsection{Evaluación del modelo}

Para que un modelo predictivo presente un buen comportamiento, sus predicciones deben ser fiables y a la vez discriminantes (Pearce \& Ferrier 2000). La fiabilidad se mide mediante la calibración/refinamiento y la discriminación sobre la bondad de las predicciones para diferenciar entre presencias y ausencias observadas. La medición del poder de discriminación se obtiene a través de la matriz de confusión. Esta matriz relaciona las predicciones de presencia/ausencia realizadas en forma correcta (True positive y True negative) así como las incorrectas (False positive y False negative). De esta matriz se obtienen índices que cuantifican el poder de discriminación de los modelos.

Estas predicciones se obtienen a través de las probabilidades de ocurrencia entregadas por el modelo. Se interpreta como predicción de ocurrencia cuando la probabilidad es mayor a un valor umbral, en caso de ser menor, se interpreta como ausencia. Los componentes de esta matriz dependerán de este valor umbral y por tanto, los valores de los índices obtenidos a partir de ella. Por defecto, en los modelos predictivos se asume un valor umbral igual a 0.5. Sin embargo, dependiendo de los objetivos del estudio es necesario optimizar este umbral (ver Pearce \& Ferrier 2000). 
El criterio utilizado para determinar el valor del umbral fue igualar la prevalencia observada de la especie con la predicha. Este criterio cobra importancia cuando la representatividad de la especie es baja, como es el caso de la mayoría de las especies de Chile (Tabla 5.1). Una vez determinado el umbral se determinaron los índices Porcentaje Correctamente Clasificado (Percent Correctly Classified-PCC), Sensibilidad (ratio entre el número de sitios positivos correctamente predichos con respecto al total), Especificidad (ratio entre el número de sitios negativos correctamente predicho con respecto al total) e índice Kappa. Este último varía entre 0.0 (no presenta capacidad de predicción) a 1.0 (presenta una capacidad perfecta).

Además se utilizaron otros índices generales los cuales no dependen del valor umbral. Estos índices son la precisión (accuracy), el estimador del error out-of-bag (OOB) y el área bajo la curva del Receiver Operator Characteristics (AUC). Este último varía entre 0.5 para modelos sin capacidad de predicción hasta 1.0 para modelos con predicciones perfectas. Una diferencia muy importante entre AUC y los indicadores anteriores es que se supone independiente del porcentaje de presencia utilizado (prevalencia)

Los valores de los índices Kappa y AUC fueron interpretados según Hosmer \& Lemeshow (2000) y Landis \& Koch (1977), respectivamente.

Los índices PCC, Sensibilidad, Especificidad, Kappa y AUC, así como la determinación del umbral óptimo, fueron determinados mediante el paquete 
CAPÍTULO 5 DISTRIBUCIÓN GEOGRÁFICA POTENCIAL.

estadístico PresenceAbsence() (Freeman \& Moisen 2008, Freeman 2009). Accuracy fue determinado según Pearce \& Ferrier (2000) y OOB mediante la librería randomForest (Breiman \& Cutler 2009). Ambas librerías están ambientadas en el lenguaje estadístico $\mathrm{R}$ versión 2.8.1 (R Development Core Team 2008).

\subsubsection{Rango de distribución geográfica}

Los rangos de distribución geográfica fueron construidos en SIG al introducir los resultados de los modelos RF para cada especie en cada tramo de río al asociar las clases REC-Chile con los respectivos códigos de los tramos de ríos. Una especie fue considerada presente cuando la probabilidad de ocurrencia es mayor que el umbral óptimo, en caso contrario se consideró como ausente. 


\subsection{RESULTADOS}

\subsubsection{Evaluación de la clasificación}

Los resultados de la evaluación de la REC-Chile bajo el objetivo de discriminación de la distribución espacial de peces nativos son satisfactorios para todos los niveles de la REC-Chile (Tabla 5.1), en donde el índice Cs presenta valores positivos para cada uno de ellos. A medida que aumenta el nivel de clasificación, y por tanto el número de clases, aumenta Cs, tendiendo a un valor similar entre los niveles 5 y 6 .

El Cs maginal ( $\Delta \mathrm{Cs})$ muestra el aumento porcentual del valor de Cs al cambiar de un nivel de clasificación a otro. $\Delta$ Cs es positivo para todos los niveles REC-Chile, por tanto, el poder de discriminación aumenta al agregar niveles de clasificación. El nivel con un mayor aumento en $\Delta \mathrm{Cs}$ es el nivel 2, luego los dichos incrementos son menores, pero siempre mayores que cero.

Las clasificaciones a posteriori, como se esperaba, tienen un mejor valor de Cs, siendo la clasificación HC la que presenta un mayor valor de Cs (Tabla 5.1). Las otras dos clasificaciones a posteriori, DC y GR, presentan valores similares de Cs que la REC-Chile. 
CAPÍTULO 5 DISTRIBUCIÓN GEOGRÁFICA POTENCIAL.

Tabla 5.1. Resultados del test clasificación de la distribución geográfica de especies ícticas bajo los 6 niveles de la REC-Chile y para las clasificaciones a posteriori Cluster Jerárquicos (HC), Cluster de distancia (DC) y clasificación geográfica (GR) $. \bar{B}=$ disimilaridad media inter-clases. $\bar{W}=$ disimilaridad media intra-clases. $C s=$ diferencia entre estas dos disimilaridades $(C s=\bar{B}-\bar{W})$ y $\Delta C s(\%)=$ aumento marginal de $C s$ al aumentar un nivel en la clasificación REC-Chile. El nivel "j" de la REC-Chile es la superposición jerárquica de los primeros "j" factores controladores.

Nivel de clasificación de la REC-Chile

\begin{tabular}{|c|c|c|c|c|c|c|c|}
\hline & & $1^{\mathrm{er}}$ nivel & $2^{d 0}$ nivel & $3^{\text {er }}$ nivel & $4^{\text {to }}$ nivel & $5^{\text {to }}$ nivel & $6^{\text {to }}$ nivel \\
\hline \multirow{5}{*}{$\begin{array}{l}\text { REC- } \\
\text { Chile }\end{array}$} & $\begin{array}{l}\mathrm{N}^{\mathrm{o}} \\
\text { clases }\end{array}$ & 5 & 18 & 40 & 61 & 86 & 105 \\
\hline & $\bar{W}$ & 0.801 & 0.722 & 0.693 & 0.648 & 0.617 & 0.606 \\
\hline & $\bar{B}$ & 0.872 & 0.867 & 0.871 & 0.860 & 0.859 & 0.854 \\
\hline & $C s$ & 0.071 & 0.145 & 0.178 & 0.213 & 0.242 & 0.248 \\
\hline & $\Delta C s(\%)$ & & 104.6 & 22.6 & 19.5 & 13.5 & 2.6 \\
\hline \multirow{2}{*}{$\mathrm{HC}$} & $C s$ & 0.449 & 0.601 & 0.703 & 0.783 & 0.852 & 0.900 \\
\hline & $\Delta C s(\%)$ & & 33.9 & 17.0 & 11.4 & 8.8 & 5.7 \\
\hline \multirow{2}{*}{$\mathrm{DC}$} & $C s$ & 0.085 & 0.194 & 0.215 & 0.256 & 0.274 & 0.285 \\
\hline & $\Delta C s(\%)$ & & 126.7 & 11.2 & 19.1 & 7.0 & 4.0 \\
\hline \multirow{2}{*}{ GR } & Cs & 0.118 & 0.228 & 0.259 & 0.279 & 0.277 & 0.276 \\
\hline & $\Delta C s(\%)$ & & 92.6 & 13.8 & 7.6 & -0.6 & -0.2 \\
\hline
\end{tabular}

Al comparar los $\Delta \mathrm{Cs}$ entre las clasificaciones, tanto a priori como a posteriori, se aprecia un incremento en todos los niveles de las clasificaciones, a excepción de la clasificación GR que presenta reducciones para los dos últimos niveles. El mayor valor de $\Delta$ Cs se produce en el segundo nivel de clasificación para las 4 clasificaciones, produciéndose mayores aumentos en DC, REC-Chile y GR, mientras que HC muestra un aumento menor. 


\subsubsection{Modelo de predicción}

El rango de las prevalencias observadas de las especies es amplio (Tabla 5.2), siendo $T$. areolatus la especie con una mayor prevalencia observada (0.369), seguida de $P$. irwini y $P$. gillissi con representatividades iguales 0.225 y 0.184 , respectivamente. Un alto porcentaje de las especies presenta una representatividad observada menor al 5\%, entre ellas destacan las especies $T$. rivulatus, $O$. ascotanensis, $O$. agassii, T. laucaensis, $B$. semotilus, $O$. laucaensis, $O$. mauleanum, $O$. piacotaensis, $O$. parinacotaensis, $T$. chungaraensis, $O$. chungaraensis con prevalencias menores al 1\%. A partir de estos resultados se obtuvo que los valores óptimos para el umbral de corte varía entre 0,64 para $P$. irwini y 0,01 para M. lapicida. 
Tabla 5.2 Información general utilizada en el modelo $\left(\mathrm{N}^{\circ}\right.$ Pres $=$ Cantidad de observaciones de presencia, $\mathrm{N}^{\circ}$ Aus: Cantidad de observaciones de ausencia, Prev. Obs= Prevalencia observada calculada como el número de observaciones de presencia divida en el total de observaciones), resultados del modelo (Prev Pred= Prevalencia predicha por el modelo de distribución espacial y valor umbral optimo calculado igualando las representatividades observadas y predichas). Estado de conservación y origen de las especies sensu Habit et al. (2006)

\begin{tabular}{l|c|c|c|c|c|cc}
\hline \multicolumn{1}{c|}{ Especie } & $\mathbf{N}^{\circ}$ Pres. & $\mathbf{N}^{\circ}$ Aus. & Prev. Obs. & Prev. Pred. & Umbral & Estado de Conservación & Origen \\
\hline \hline B. australis & 77 & 408 & 0.159 & 0.155 & 0.215 & Vulnerable & Nativa \\
B. bullocki & 10 & 475 & 0.021 & 0.019 & 0.035 & Vulnerable & Endémica \\
B. maldonadoi & 51 & 434 & 0.105 & 0.118 & 0.145 & Peligro de extinción & Endémica \\
B. microlepidotus & 19 & 466 & 0.039 & 0.039 & 0.415 & Peligro de extinción & Endémica \\
B. semotilus & 2 & 483 & 0.004 & 0.004 & 0.365 & Peligro de extinción & Endémica \\
Ch. australe & 16 & 469 & 0.033 & 0.029 & 0.050 & Vulnerable & Endémica \\
Ch. galusdae & 64 & 421 & 0.132 & 0.136 & 0.500 & Vulnerable & Endémica \\
Ch. pisciculus & 19 & 466 & 0.039 & 0.039 & 0.305 & Vulnerable & Endémica \\
D. camposensis & 28 & 457 & 0.058 & 0.064 & 0.115 & Peligro de extinción & Endémica \\
D. chilensis & 6 & 479 & 0.012 & 0.008 & 0.145 & Peligro de extinción & Endémica \\
D. nahuelbutaensis & 45 & 440 & 0.093 & 0.062 & 0.020 & Peligro de extinción & Endémica \\
G. maculatus & 77 & 408 & 0.159 & 0.080 & 0.150 & Vulnerable & Nativa \\
G. australis & 41 & 444 & 0.085 & 0.165 & 0.190 & Vulnerable & Nativa \\
G. platei & 8 & 477 & 0.016 & 0.000 & 0.505 & Vulnerable & Endémica \\
M. lapicida & 7 & 478 & 0.014 & 0.014 & 0.010 & Indeterminado & Endémica \\
N. inermis & 18 & 467 & 0.037 & 0.037 & 0.100 & Peligro de extinción & Endémica \\
O. agassii & 3 & 482 & 0.006 & 0.006 & 0.435 & Indeterminado & Nativa \\
\hline
\end{tabular}


Tabla 5.2 Información general utilizada en el modelo $\left(\mathrm{N}^{\circ}\right.$ Pres $=$ Cantidad de observaciones de presencia, $\mathrm{N}^{\circ}$ Aus: Cantidad de observaciones de ausencia, Prev. Obs= Prevalencia observada calculada como el número de observaciones de presencia divida en el total de observaciones), resultados del modelo (Prev Pred= Prevalencia predicha por el modelo de distribución espacial y valor umbral optimo calculado igualando las representatividades observadas y predichas). Estado de conservación y origen de las especies sensu Habit et al. (2006)

\begin{tabular}{l|c|c|c|c|c|cc}
\hline \multicolumn{1}{c}{ Especie } & $\mathbf{N}^{\circ}$ Pres. & $\mathbf{N}^{\circ}$ Aus. & Prev. Obs. & Prev. Pred. & Umbral & Estado de Conservación & Origen \\
\hline \hline O. ascotanensis & 3 & 482 & 0.006 & 0.006 & 0.385 & Peligro de extinción & Endémica \\
O. chungaraensis & 1 & 484 & 0.002 & 0.000 & 0.540 & Peligro de extinción & Endémica \\
O. mauleanum & 1 & 484 & 0.002 & 0.002 & 0.415 & Vulnerable & Endémica \\
O. parinacotaensis & 1 & 484 & 0.002 & 0.000 & 0.505 & Peligro de extinción & Endémica \\
O. piacotaensis & 1 & 484 & 0.002 & 0.000 & 0.530 & Indeterminado & Endémica \\
O. laucaensis & 1 & 484 & 0.002 & 0.000 & 0.525 & Peligro de extinción & Endémica \\
P. gillissi & 89 & 396 & 0.184 & 0.188 & 0.220 & Vulnerable & Endémica \\
P. irwini & 109 & 376 & 0.225 & 0.229 & 0.640 & Peligro de extinción & Endémica \\
P. melanops & 9 & 476 & 0.019 & 0.023 & 0.095 & Peligro de extinción & Endémica \\
P. trucha & 83 & 402 & 0.171 & 0.169 & 0.240 & Vulnerable & Nativa \\
T. areolatus & 179 & 306 & 0.369 & 0.369 & 0.255 & Vulnerable & Nativa \\
T. chiltoni & 9 & 476 & 0.019 & 0.019 & 0.050 & Peligro de extinción & Endémica \\
T. chungaraensis & 1 & 484 & 0.002 & 0.000 & 0.530 & Peligro de extinción & Endémica \\
T. laucaensis & 2 & 483 & 0.004 & 0.006 & 0.315 & Peligro de extinción & Endémica \\
T. rivulatus & 4 & 481 & 0.008 & 0.010 & 0.300 & Rara & Endémica \\
\hline
\end{tabular}




\subsubsection{Evaluación del modelo}

La evaluación de los modelos de predicción se centró en los índices AUC y Kappa. Para el índice AUC, los modelos presentan, en general, un buen comportamiento abarcando valores entre 1 y 0.697 con valor medio igual a 0.916. Según la interpretación realizada por Hosmer \& Lemeshow (2000) un $56 \%$ de los modelos presenta una discriminación "outstanding”, un $31 \%$ presenta una discriminación "excelente", un 9\% presenta un poder de discriminación "aceptable" y solo una especie $(3 \%)$ presenta un poder de discriminación "pobre".

En el índice Kappa, un 25\% de los modelos presentan un nivel de concordancia "Casi perfecto", un 9\% "bueno", un 31\% "moderado", un 16\% “discreto" y un 19\% "pobre" (sensu Landis \& Koch 1977). Cuando el modelo presenta una concordancia extrema (Casi perfecto o pobre) el comportamiento del modelo es errático, por lo tanto es aconsejable no considerarlo en los futuros análisis.

Recordando que el índice Kappa depende del valor umbral, el cual fue determinado igualando la prevalencia observada con la predicha, es posible asociar la presencia de valores extremos de este índice con la prevalencia (Mouton et al. 2009). Para prevalencias menores a 1.6\% la mayoría de los modelos presentan valores extremos de Kappa. Para prevalencias mayores a $1.6 \%$ los valores Kappa son más “centrados”. En consecuencia, de los 32 modelos originales se han eliminado del análisis 14 de ellos, resultando 18 modelos confiables (Tabla 5.3). 
Tabla 5.3: Evaluación del modelo predictivo Random Forest para cada una de las especies ícticas nativas de Chile. OOB error es el estimador Out-of-bag del modelo Random Forest. AUC es el área bajo la curva del Receiver operating Characteristic. Los parámetros Precisión, Porcentaje correctamente clasificados (PCC), sensibilidad (Sensib.) y Especificidad (Especif.) son determinados mediante la matriz de confusión (Pearce \& Ferrier 2000). Los índices AUC y Kappa presentan una interpretación según el valor de acuerdo a Hosmer \& Lemeshow (2000) y Landis \& Koch (1977) respectivamente. La columna Exclusión hace mención a si el modelo fue eliminado o no de posteriores análisis según criterios explicados en el texto.

\begin{tabular}{lcccccccccc}
\hline \multicolumn{1}{c}{ Especie } & $\begin{array}{c}\text { OOB } \\
\text { error }\end{array}$ & AUC & $\begin{array}{c}\text { Hosmer } \boldsymbol{\text { Lemeshow }} \\
\text { Kappa }\end{array}$ & $\begin{array}{c}\text { Landis \& } \\
\text { Koch }\end{array}$ & Precisión & PCC & Sensib & Especif & Excl. \\
\hline \hline B.australis & $16.49 \%$ & 0.836 & Excellent & 0.501 & moderate & 0.837 & 0.868 & 0.571 & 0.924 & No \\
B.bullocki & $2.06 \%$ & 0.757 & Acceptable & 0.302 & Fair & 0.979 & 0.973 & 0.300 & 0.987 & No \\
B.maldonadoi & $11.55 \%$ & 0.870 & Excellent & 0.521 & moderate & 0.885 & 0.905 & 0.608 & 0.940 & No \\
B.microlepidotus & $2.06 \%$ & 0.998 & Outstanding & 0.836 & almost perfect & 0.979 & 0.988 & 0.842 & 0.994 & No \\
B.semotilus & $0.41 \%$ & 1.000 & Outstanding & 1.000 & almost perfect & 0.996 & 1.000 & 1.000 & 1.000 & Sí \\
Ch.australe & $3.30 \%$ & 0.739 & Acceptable & 0.312 & Fair & 0.967 & 0.959 & 0.312 & 0.981 & No \\
Ch.galusdae & $13.81 \%$ & 0.926 & Outstanding & 0.609 & substantial & 0.862 & 0.909 & 0.672 & 0.945 & No \\
Ch.pisciculus & $1.65 \%$ & 0.998 & Outstanding & 0.890 & almost perfect & 0.984 & 0.992 & 0.895 & 0.996 & No \\
D.camposensis & $6.19 \%$ & 0.959 & Outstanding & 0.549 & moderate & 0.938 & 0.948 & 0.607 & 0.969 & No \\
D.chilensis & $1.24 \%$ & 0.986 & Outstanding & 0.394 & Fair & 0.988 & 0.988 & 0.333 & 0.996 & Sí \\
D.nahuelbutaensis & $9.48 \%$ & 0.790 & Acceptable & 0.265 & Fair & 0.905 & 0.895 & 0.267 & 0.959 & No \\
G.australis & $8.25 \%$ & 0.879 & Excellent & 0.428 & moderate & 0.918 & 0.913 & 0.463 & 0.955 & No \\
G.maculatus & $12.58 \%$ & 0.843 & Excellent & 0.536 & moderate & 0.874 & 0.874 & 0.623 & 0.922 & No \\
G.platei & $1.65 \%$ & 0.966 & Outstanding & 0.000 & Poor & 0.984 & 0.984 & 0.000 & 1.000 & Sí \\
M.lapicida & $1.44 \%$ & 0.697 & Poor & 0.275 & Fair & 0.986 & 0.979 & 0.286 & 0.990 & Sí \\
N.inermis & $3.71 \%$ & 0.833 & Excellent & 0.481 & moderate & 0.963 & 0.963 & 0.500 & 0.981 & No \\
\hline
\end{tabular}




\section{CAPÍTULO 5 DISTRIBUCIÓN GEOGRÁFICA POTENCIAL.}

Tabla 5.3: Evaluación del modelo predictivo Random Forest para cada una de las especies ícticas nativas de Chile. OOB error es el estimador Out-of-bag del modelo Random Forest. Auc es el área bajo la curva del Receiver operating Characteristic. Los parámetros Precisión, Porcentaje correctamente clasificados (PCC), sensibilidad (Sensib.) y Especificidad (Especif.) son determinados mediante la matriz de confusión (Pearce \& Ferrier 2000). Los índices AUC y Kappa presentan una interpretación según el valor de acuerdo a Hosmer \& Lemeshow (2000) y Landis \& Koch (1977) respectivamente. La columna Exclusión hace mención a si el modelo fue eliminado o no de posteriores análisis según criterios explicados en el texto.

\begin{tabular}{lcccccccccc}
\hline \multicolumn{1}{c}{ Especie } & $\begin{array}{c}\text { OOB } \\
\text { error }\end{array}$ & AUC & $\begin{array}{c}\text { Hosmer \& } \\
\text { Lemeshow }\end{array}$ & Kappa & $\begin{array}{c}\text { Landis \& } \\
\text { Koch }\end{array}$ & Precisión & PCC & Sensib & Especif & Excl. \\
\hline \hline O.agassii & $0.82 \%$ & 1.000 & Outstanding & 1.000 & almost perfect & 0.992 & 1.000 & 1.000 & 1.000 & Sí \\
O.ascotanensis & $0.21 \%$ & 1.000 & Outstanding & 1.000 & almost perfect & 0.998 & 1.000 & 1.000 & 1.000 & Sí \\
O.chungaraensis & $0.41 \%$ & 0.999 & Outstanding & 0.000 & Poor & 0.996 & 0.998 & 0.000 & 1.000 & Sí \\
O.laucaensis & $0.21 \%$ & 1.000 & Outstanding & 1.000 & almost perfect & 0.998 & 1.000 & 1.000 & 1.000 & Sí \\
O.mauleanum & $0.21 \%$ & 0.993 & Outstanding & 0.000 & Poor & 0.998 & 0.998 & 0.000 & 1.000 & Sí \\
O.parinacotaensis & $0.41 \%$ & 0.999 & Outstanding & 0.000 & Poor & 0.996 & 0.998 & 0.000 & 1.000 & Sí \\
O.piacotaensis & $0.41 \%$ & 0.999 & Outstanding & 0.000 & Poor & 0.996 & 0.998 & 0.000 & 1.000 & Sí \\
P.gillissi & $6.60 \%$ & 0.963 & Outstanding & 0.807 & almost perfect & 0.934 & 0.942 & 0.843 & 0.965 & No \\
P.irwini & $15.88 \%$ & 0.897 & Excellent & 0.581 & moderate & 0.841 & 0.854 & 0.679 & 0.904 & No \\
P.melanops & $1.86 \%$ & 0.857 & Excellent & 0.490 & moderate & 0.981 & 0.979 & 0.556 & 0.987 & No \\
P.trucha & $15.26 \%$ & 0.849 & Excellent & 0.584 & moderate & 0.847 & 0.882 & 0.651 & 0.930 & No \\
T.areolatus & $21.03 \%$ & 0.867 & Excellent & 0.628 & substantial & 0.790 & 0.827 & 0.765 & 0.863 & No \\
T.chiltoni & $2.06 \%$ & 0.812 & Excellent & 0.547 & moderate & 0.979 & 0.984 & 0.556 & 0.992 & No \\
T.chungaraensis & $0.41 \%$ & 0.999 & Outstanding & 0.000 & Poor & 0.996 & 0.998 & 0.000 & 1.000 & Sí \\
T.laucaensis & $0.62 \%$ & 0.999 & Outstanding & 0.799 & substantial & 0.994 & 0.998 & 1.000 & 0.998 & Sí \\
T.rivulatus & $0.82 \%$ & 1.000 & Outstanding & 0.888 & almost perfect & 0.992 & 0.998 & 1.000 & 0.998 & Sí \\
\hline
\end{tabular}




\subsubsection{Importancia de las variables}

La importancia de las variables se analizó para los 18 modelos que presentan un buen comportamiento estadístico, es decir, para aquellos que fueron excluidos (Tabla 5.4). Para cada uno de estos modelos la importancia fue escalada, es decir, los valores de la importancia fueron divididas por el máximo de cada modelo. Con esto, la variable con mayor importancia tendrá un valor igual a 1 , a medida que el valor disminuye la importancia de la variable lo hace también. La importancia conjunta se obtuvo sumando la importancia de cada variable en los 18 modelos. Se observa que el factor CL (Clima) es la variable más determinante en la distribución espacial de las especies íctica en análisis, seguido estrechamente del factor OF (Origen del Flujo), US (Uso de Suelo), PRS (Posición relativa de subsubcuenca), G (Geología) y por último P (Pendiente del tramo).

\begin{tabular}{|c|c|c|c|c|c|c|}
\hline \multirow{3}{*}{$\begin{array}{l}\text { Tabla } 5.4 \text {. } \\
\text { factores cont } \\
\text { uno de los } \\
\text { Origen de Flu } \\
\text { Subsubcuenca; } \\
\text { tramo } \\
\text { Especie }\end{array}$} & \multicolumn{6}{|c|}{$\begin{array}{l}\text { Importancia escalada y conjunta de los } \\
\text { oladores de la REC-Chile utilizados en cada } \\
\text { odelos Random Forests. CL = Clima; OF = } \\
\text { o; G = Geología; PRS = Posición Relativa de } \\
\text { US = Uso del Suelo y P = Pendiente del }\end{array}$} \\
\hline & \multicolumn{6}{|c|}{ Factor controlador REC-Chile } \\
\hline & $\mathbf{C L}$ & OF & $\mathbf{G}$ & PRS & US & $\mathbf{P}$ \\
\hline B.australis & 0.807 & 0.846 & 0.768 & 1.000 & 0.926 & 0.620 \\
\hline B.bullocki & 0.343 & 0.873 & 0.614 & 0.512 & 0.977 & 1.000 \\
\hline B.maldonadoi & 0.784 & 0.833 & 0.700 & 1.000 & 0.984 & 0.833 \\
\hline B.microlepidotus & 1.000 & 0.954 & 0.790 & 0.777 & 0.927 & 0.643 \\
\hline Ch.australe & 1.000 & 0.679 & 0.733 & 0.261 & -0.251 & 0.685 \\
\hline Ch.galusdae & 1.000 & 0.856 & 0.714 & 0.861 & 0.643 & 0.715 \\
\hline Ch.pisciculus & 1.000 & 0.798 & 0.769 & 0.743 & 0.660 & 0.318 \\
\hline D.camposensis & 0.526 & 1.000 & 0.531 & 0.902 & 0.703 & 0.879 \\
\hline D.nahuelbutaensis & 0.701 & 0.811 & 0.842 & 0.646 & 1.000 & 0.112 \\
\hline G.australis & 1.000 & 0.789 & 0.599 & 0.923 & 0.702 & 0.333 \\
\hline
\end{tabular}


CAPÍTULO 5 DISTRIBUCIÓN GEOGRÁFICA POTENCIAL.

Tabla 5.4. Importancia escalada y conjunta de los factores controladores de la REC-Chile utilizados en cada uno de los modelos Random Forests. $\mathrm{CL}=\mathrm{Clima} ; \mathrm{OF}=$ Origen de Flujo; $\mathrm{G}=$ Geología; $\mathrm{PRS}=$ Posición Relativa de Subsubcuenca; US = Uso del Suelo y $\mathrm{P}=$ Pendiente del tramo

\begin{tabular}{lcccccc}
\hline \multirow{2}{*}{ Especie } & \multicolumn{7}{c}{ Factor controlador REC-Chile } \\
\cline { 2 - 7 } & CL & OF & G & PRS & US & P \\
\hline \hline G.maculatus & 0.908 & 0.905 & 0.812 & 1.000 & 0.716 & 0.677 \\
N.inermis & 0.631 & 0.363 & 0.342 & 0.704 & 1.000 & 0.573 \\
P.gillissi & 0.925 & 1.000 & 0.850 & 0.953 & 0.882 & 0.839 \\
P.irwini & 0.944 & 0.931 & 0.899 & 0.853 & 1.000 & 0.712 \\
P.melanops & 0.645 & 0.438 & 0.771 & 0.540 & 1.000 & 0.550 \\
P.trucha & 1.000 & 0.878 & 0.899 & 0.807 & 0.903 & 0.853 \\
T.areolatus & 0.979 & 1.000 & 0.976 & 0.997 & 0.971 & 0.809 \\
T.chiltoni & 0.707 & 0.627 & 1.000 & 0.514 & 0.478 & 0.377 \\
\hline Importancia & 14.9 & 14.6 & 13.6 & 14.0 & 14.2 & 11.5 \\
conjunta escalada & 14.9 & & & & & \\
\hline
\end{tabular}

\subsubsection{Distribución geográfica}

En las Figuras 5.1 a 5.18 (anexos de capítulo) se muestran las probabilidades de ocurrencia y la distribución geográfica (presencia/ausencia) para los 18 modelos que fueron seleccionados en el apartado anterior. En general, se aprecia que la distribución de las especies abarca principalmente la zona central de Chile. Para la mayoría de estas especies, la probabilidades de ocurrencia en el norte es baja $(<10 \%)$ y por tanto suele ser considerado como ausencia, dependiendo del valor umbral de corte para cada modelo. De igual forma, los tramos altos de cordillera parecieran no ser aptos para las especies nativas representadas en estos modelos. 


\subsection{DISCUSIÓN}

\subsubsection{Evaluación REC-Chile}

El test de evaluación muestra que la REC-Chile pareciera ser un sistema de clasificación adecuado para capturar los patrones de distribución geográfica de los peces nativos de Chile. El aumento de Cs a medida que aumenta el nivel REC-Chile y por tanto, (el número de clases) se produce conforme al aumento de la homogeneidad intra-clases señalada por Bryan (2006). El aumento marginal de la homogeneidad $(\Delta \mathrm{Cs})$ también se comporta según Bryan (2006), en donde el aumento de homogeneidad es brusco en grandes escalas y disminuye a medida que la escala disminuye. De acuerdo a las propiedades de la REC-Chile, ésta tiene la flexibilidad de cambiar de escala fácilmente (Peredo-Parada et al. 2010) y por tanto es posible determinar de una forma fácil cuál es la mejor escala de trabajo de acuerdo a la relación complejidad de la clasificación v/s homogeneidad de las clases.

A nivel global, el poder de discriminación de las clasificaciones a posteriori son mejores que la clasificación REC-Chile. Dentro de éstas, la clasificación realizada mediante cluster jerárquico $(\mathrm{HC})$ presenta valores de Cs muy superior a la REC-Chile, sin embargo, las otras dos clasificaciones $a$ posteriori (DC y GR) presentan un comportamiento mejor pero no tan destacado respecto a la REC-Chile. Esto conlleva a suponer que hay una fuerte orientación geográfica norte-sur en la distribución de los peces

nativos de Chile, debido a que estas clasificaciones (DC y GR) son construidas a partir de proximidades. 
El nivel de discriminación de la REC-Chile es levemente superior que los obtenidos a los obtenidos en otras clasificaciones a priori para determinar patrones de distribución geográfica de especies acuáticas. En macroinvertebrados, los autores Waite et al. (2000), Sandin \& Johnson (2000), Marchant et al. (2000), Rabeni \& Doisy (2000), Feminella (2000), Gerritsen et al (2000), Hawkins \& Vinson (2000) y Johnson (2000) obtuvieron valores de Cs por debajo de 0.16 (más detalles ver Hawkins et al. 2000). En peces la situación no es distinta, McCormick et al. (2000) obtiene valores de $\mathrm{Cs}=0.071$., mientras la REC-Chile obtuvo valores comprendido entre 0.071 y 0.248 , dependiendo del nivel de clasificación (Tabla 5.1).

Una de las posibles razones por la cuales la REC-Chile obtiene mejores resultados es su estructura jerárquica basada en el clima y la topografía (Peredo-Parada et al. 2010) que permite una mejor clasificación de la variabilidad geográfica de la especies acuáticas (Hawkins et al. 2000, Snelder et al. 2004), como se aprecia en el aumento del Cs desde el segundo (Origen de Flujo) al tercer nivel (Geología). Similar, situación obtuvo Chapin (2006) al utilizar diferentes clasificaciones a diversas escalas para clasificar peces en E.E.U.U. obteniendo valores de $\mathrm{Cs}=0.20$

Sin embargo, la utilización de una clasificación jerárquica no asegura el éxito en la clasificación de la variabilidad, Snelder et al. (2004) clasificó macroinvertebrados en Nueva Zelanda mediante la River Environmental Classification (REC) a un cuarto nivel de clasificación (mesoescala), obteniendo valores bajos de clasificación $(\mathrm{Cs}=0.09)$. A pesar de estos bajos 
CAPITULO 5 DISTRIBUCIÓN GEOGRÁFICA POTENCIAL. resultados, la clasificación a priori REC presentó mejores resultados que las otras clasificaciones a priori no jerárquicas implementadas en Nueva Zelanda y que fueron evaluadas en dicho estudio.

La REC-Chile, al igual que la REC, está basada en la suposición de que las características ecológicas locales pueden ser explicadas por características ambientales a gran escala (Peredo-Parada et al. 2010) por tanto, considera efectos locales, los cuales podrían aumentar el nivel de clasificación de especies acuáticas (Hawkins et al. 2000).

Otra razón por la cual la REC-Chile aumentaría su poder de discriminación sobre distribución de comunidades acuáticas es la posibilidad de propagar hacia aguas abajo las características más influyentes de cada factor controlador (River continuum), permitiendo representar los cambios acumulados que se producen aguas abajo en un río (Snelder \& Biggs 2002).

\subsubsection{Modelo de predicción}

Los resultados indican que es posible determinar las distribución geográfica potencial de especies ícticas mediante la técnica Random Forests. Entre las ventajas de utilizar esta técnica destacan: 1) Es capaz de capturar las relaciones no lineales entre los factores ambientales (predictores) y la distribución geográfica potencial (variables respuesta) 2) Tiene la posibilidad de implementar estos modelos en zonas con poca información, ya que RF no hace suposiciones sobre la distribución de los predictores ni en las variables de salida del modelo, lo que permite manipular situaciones 
CAPÍTULO 5 DISTRIBUCIÓN GEOGRÁFICA POTENCIAL.

en las cuales el número de predictores excede ampliamente el número de observaciones (Cutler et al. 2007); 3) Permite la implementación utilizando las clases REC-Chile como predictores.

Los modelos de las especies ícticas entregan bajos valores del estimador OOB, lo cual puede interpretarse como un buen comportamiento general del modelo, sin embargo, los índices Kappa y AUC indican un comportamiento pobre o errático. Este comportamiento errático está asociado a modelos con bajas prevalencias. Para eliminar este comportamiento Buisson et al. (2008) aconsejan utilizar aquellas especies que tengan una prevalencia mayor al 5\%. Sin embargo, la mayoría de las especies ícticas está por debajo de dicha prevalencia. Por lo tanto, si consideramos como prevalencia mínima aquella propuesta por Buisson et al. (2008) solo en un 35\% de las especies podría desarrollarse un modelo de distribución espacial, lo que supondría solo 11 modelos en lugar de los 18 realizados.

Entre las causas por la cuales las especies ícticas presentan baja prevalencia en los datos disponibles se encuentran 1) la escasez de información íctica disponible en Chile (Peredo-Parada et al. 2009); 2) el alto grado de endemismo (Habit et al. 2006); y 3) su deteriorado estado de conservación (Habit et al. 2006, Vila et al. 1999).

Por estas razones, la prevalencia mínima se eligió como aquella en la cual el índice AUC fuese "aceptable" y el del Kappa "moderado" (sensu Hosmer \& Lemeshow 2000 y Landis \& Koch 1977, respectivamente). Asimismo, para evitar comportamientos erráticos en la discriminación de ausencia/presencia se eliminaron aquellos modelos que presentan valores extremos de Kappa 
CAPÍTULO 5 DISTRIBUCIÓN GEOGRÁFICA POTENCIAL. (cercanos a 0 y a 1) y que tuviesen una baja prevalencia. Los 18 modelos restantes presentan valores buenos de AUC y Kappa con comportamientos más estables. Los modelos excluidos resultaron ser mayoritariamente asociados a especies de alto endemismo en el norte de Chile.

Los umbrales que determinan si la probabilidad de ocurrencia corresponde a presencia o ausencia, resultaron ser mayoritariamente bajo el valor 0.5 que es asumido por defecto en los modelos predictivos. En caso de haber mantenido el valor por defecto del RF, la representación dicotómica de presencia/ausencia hubiese castigado en exceso la probabilidad de ocurrencia de muchas especies considerándolas como ausentes en la totalidad de los ríos de Chile.

Existen diversos criterios para determinar el valor umbral (ver Freeman \& Moisen 2008), en los cuales se tiene en cuenta la naturaleza de la especie, evaluación de costos de falsas predicciones o cantidad de datos. Esto tiene implicaciones directas de acuerdo al objetivo de los modelos de predicción. Hawkins et al. (2000) señalan que si se quieren definir zonas de conservación de la especies, entonces el umbral debe ser bajo, para aumentar la presencia de la especie, es decir, bastaría con una baja probabilidad de ocurrencia para indicar que la especie se considere como presente. En caso de querer determinar zonas de relocalización para una especie el umbral debe ser alto para asegurar el éxito de ésta.

Para las 18 especies nativas analizadas la importancia conjunta de los predictores indica que los factores controladores que más influyen sobre la distribución geográfica son el Clima y el Origen Flujo, equivalentes a los 
CAPÍTULO 5 DISTRIBUCIÓN GEOGRÁFICA POTENCIAL.

dos primeros factores controladores de la REC-Chile. Éstos actúan a una gran escala espacial, lo cual pareciera concordar en que el clima y el aislamiento biogeográfico (recordar que el factor Origen de Flujo está asociado a la topografía del terreno) son los principales agentes en el alto endemismo (Vila et al. 1999) y por tanto en su distribución geográfica

En relación a la distribución geográfica, ésta muestra grandes zonas geográficas en las cuales existen tramos de ríos sin información, debido a que las clases REC-Chile asociado a estos tramos no cuentan con ningún punto de muestreo. Estas zonas demuestran lo expuesto por Peredo-Parada et al. (2009) referente a la fragmentación de la distribución espacial de la información y que ésta está asociadas a zonas restringidas a centros de investigación.

La utilización conjunta de un modelo de distribución espacial de las especies ícticas y una sistema de clasificación, como la REC-Chile, permiten definir zonas de conservación y de relocalización de las especies, variando los niveles de umbrales óptimos según lo propuesto por Hawkins et al. (2000). Además, estas distribuciones geográficas pueden ser utilizadas como complemento a la información necesaria para los estudios de caudales ecológicos, planes de restauración fluvial, etc.

Esto constituye a la REC-Chile, en conjunto a los MDE, en una herramienta a tener en cuenta al momento de realizar planes de gestión integral de cuencas en donde la conservación de las especies es de gran importancia. 


\subsection{CONCLUSIONES}

Los resultados del índice de robustez de la clasificación (Cs) indican que la REC-Chile es capaz de lograr resultados aceptables para la discriminación de la distribución espacial de los peces nativos de Chile.

Los modelos de distribución espacial de las especies logran buenos resultados en su comportamiento general; sin embargo, para aquellos con muy bajas prevalencias los índices AUC y Kappa indican un comportamiento errático del modelo.

Los modelos obtenidos, basados en las clases REC-Chile como predictoras, en general no tienen un comportamiento excelente, pero algunos modelos presentan un comportamiento satisfactorio, lo cual podría concluirse que el utilizar este tipo de variables predictoras puede ser un primer paso para suplir la falta de información íctica disponible.

Por último, se resalta que la utilización de la REC-Chile es una herramienta importante a tener en cuenta para la gestión integrada de las cuencas. 


\subsection{BIBLIOGRAFÍA}

AGUILAR A, M GEVREY, P YOUNG-SEK, L PUY \& S LEK. 2003. Modelling the factors that influence fish guilds composition using a back-propagation network: Assessment of metrics for indices of biotic integrity. Ecological Modelling 160: 281-290.

AUSTIN M. 2007. Species distribution models and ecological theory: a critical assessment and some possible new approaches. Ecological Modelling 200: 1-19.

BREIMAN L. 2001. Random Forests. Machine Learning, 45: 32- 5 pp.

BREIMAN L \& A CUTLER. 2009. Breiman and Cutler's random forests for classification and regression. $\mathrm{R}$ package version 4.5-30. URL. http://statwww.berkeley.edu/users/breiman/RandomForests.

BRYAN B. 2006. Synergistic techniques for better understanding and classifying the environmental structure of landscape. Environmental Management 37(1): 126140 .

BROSSE S, C ARBUCKLE \& C TOWNSEND. 2003. Habitat scale and biodiversity: Influence of catchment, stream reach and bedform scales on local inveertebrate diversity. Biodiversity and Conservation. 12: 2057-2075.

BUISSON L, L BLANC \& G GRENOUILLET. 2008. Modelling stream fish species distribution in a river network: the relative effects of temperature versus physical factors. Ecology of Freshwater Fish 17: 244-257.

\section{CHAPIN 2006}

CUTLER D, T EDWARDS Jr., K BEARD, A CUTLER, K. HESS, J GIBSON \& J LAWLER. 2007. Random Forests for classification in ecology. Ecology 88 (11): 2783-2792.

DOMÍNGUEZ-DOMÍNGUEZ O, E MARTÍNEZ-MEYER, L ZAMBRANO \& G PÉREZ-PONCE DE LEÓN. 2006. Using ecological-niche modeling as a conservation tool for freshwater species: live-bearing fishes in central Mexico. Conservation Biology 20 (6):1730-1739.

DYER B. 2000. Revisión sistemática y biogeográfica de los peces dulceacuícolas de chile. Estudios Oceanológicos 19: 77 - 98. 
CAPITULO 5 DISTRIBUCIÓN GEOGRÁFICA POTENCIAL. ELITH J, C GRAHAM, R ANDERSON, M DUDÍK, S FERRIER, A GUISAN, R HIJMANS, FHUETTMANN, J LEATHWICK, A LEHMANN, J LI, L LOHMANN, B LOISELLE, G MANION, C MORITZ, M NAKAMURA, Y NAKAZAWA, J OVERTON, A TOWNSEND, S PHILLIPS, K RICHARDSON, R SCACHETTI-PEREIRA, R SCHAPIRE, J SOBERÓN, S WILLIAMS, M WISZ \& N ZIMMERMANN. 2006. Novel methods improve prediction of species' distributions from occurrence data. Ecography 29: 129-151.

FEMINELLA J 2000. Correspondence between stream macroinvertebrate assemblages and 4 ecoregions of the southeastern USA. Journal of the North American Benthological Society 19: 442-461.

FREEMAN E. 2009. Presence-Absence Model Evaluation. R package version 1.1.3. URL http://CRAN.R-project.org/

FREEMAN E \& G MOSIEN. 2008. PresenceAbsence: An R Package for Presence Absence Analysis. Journal of Statistical Software. 23 (11).

FRISSELL C, WJ LISS, CE WARREN \& MD HURLEY 1986. A hierarchical framework for Stream Habitat Classification: Viewing Streams in Watershed context. Environmental Management 10:199-214.

GERRITSEN J, M BARBOUR \& K KING. 2000. Apples, oranges, and ecoregions: on determining pattern in aquatic assemblages. Journal of the North American Benthological Society 19:487- 496.

HABIT E, B DYER \& I VILA. 2006. Estado de conocimiento de los peces dulceacuícolas de Chile. Gayana. 70. (1): 100-112.

HABIT E, M BELK \& O PARRA. 2007. Response of the riverine fish community to the construction and operation of a diversion hydropower plant in central Chile. Aquatic Conservation: Marine And Freshwater Ecosystems 17: 37-49

HAWKINS C \& M VINSON. 2000. Weak correspondence between landscape classifications and stream invertebrate assemblages: implications for bioassessment. Journal of the North American Benthological Society 19:501-517.

HAWKINS C, R NORRIS, J GERRITSEN, R HUGHES, S JACKSON, R JOHNSON \& J STEVENSON. 2000. Evaluation of the use of landscape classifications for the predictios of freshwater biota: synthesis and recommendations. Journal of American Benthological Society. 19 (3): 541-556.

HERMOSO V, S LINKE \& J PRENDA. 2009. Identifying priority sites for the conservation of freshwater fish biodiversity in a Mediterranean basin with a high degree of threatened endemics. Hydrobiologia 623:127-140. 
CAPITULO 5 DISTRIBUCIÓN GEOGRÁFICA POTENCIAL.

HOSMER D, LEMESHOW S. 2000. Applied Logistic Regression, 2nd ed. John Wiley \& Sons, New York, USA.392.

JOHNSON R. 2000. Spatial congruence between ecoregions and littoral macroinvertebrate assemblages. Journal of North American Benthological Society 19:475-486.

LANDIS J \& G KOCH (1977) The measurement of observer agreement for categorical data. Biometrics 33:159-174.

LEATHWICK JR, J ELITH \& T HASTIE. 2006. Comparative performance of generalized additive models and multivariate adaptive regression splines for statistical modelling of species distributions. Ecological Modelling. 199: 188-196.

LEGENDRE P \& L LEGENDRE. 1998. Numerical Ecology. (Eds Elsevier) 2nd edition, Amsterdam $853 \mathrm{pp}$.

LENG W, H HE, R BU, L DAI, Y HU, X WANG. 2008. Predicting the distributions of suitable habitat for three larch species under climate warming in Northeastern China. Forest Ecology and Management 254: 420-428.

LOBO J, A JIMÉNEZ-VALVERDE \& R REAL. 2008. AUC: a misleading measure of the performance of predictive distribution models. Global Ecology and Biogeography. 17: 145-151.

MARCHANT R, F WELLS \& P NEWALL. 2000. Assessment of an ecoregion approach for classifying macroinvertebrate assemblages from streams in Victoria, Australia. Journal of the North American Benthological Society 19: 497-500.

MARMION M, M LUOTO, R HEIKKINEN \& W THUILLER. 2008. The performance of state-of-the-art modelling techniques depends on geographical distribution on species. Ecological Modelling. 220: 3512-3520.

McCORMICK F ,D PECK \& D. LARSEN. 2000. Comparison of geographic classification schemes for Mid-Atlantic stream fish assemblages. Journal of the North American Benthological Society 19: 385- 404.

McNYSET KM. 2005. Use of ecological niche modelling to predict distributions of freshwater fish species in Kansas. Ecology of Freshwater Fish 14 : 243-255.

MUGODO J, M KENNARD, P LISTON, S NICHOLS, S LINKE, R NORRIS \& M LINTERMANS. 2006. Local stream habitat variables predicted from catchment scale characteristics are useful for predicting fish distribution. Hydrobiologia. 572: 59-70. 
CAPITULO 5 DISTRIBUCIÓN GEOGRÁFICA POTENCIAL. OAKES RM, KB GIDO, JA FALKE, JDOLDEN \& BL BROCK. 2005. Modelling of stream fishes in the Great Plains, USA.Ecology of Freshwater Fish 14: 361-374.

OLDEN J \& J JACKSON. 2002. A comparision of statistical approaches for modelling fish specie distributions. Freshwater Biology 47:1976-1995.

OLDEN J, N POFF \& B BLEDSOE. 2006. Incorporating ecological knowledge into ecoinformatics: An example of modeling hierarchically structured aquatic communities with neural networks. Ecological Informatics 1: 33-42.

OLDEN J, J LAWLER, N POFF. 2008. Machine learning methods without tears: a primer for ecologists. Quart. Rev. Biol. 83:171-193.

ORREGO R, S MARSHALL, R BARRA, G CHIANG \& JF GAVILÁN. 2009. Patterns of fish community composition along a river affected by agricultural and urban disturbance in south-central Chile. Hidrobiología 620:35-46.

OYANEDEL J, C VEGA-RETTER, S SCOTT, L HINOJOSA \& R RAMOSJILIBERTO. 2008. Finding patterns of distribution for freshwater phytoplankton, zooplankton and fish, by means of parsimony analysis of endemicity. Revista Chilena de Historia Natural 81: 185-203.

PARDO R, I VILA \& JJ CAPELLA. 2009. Competitive interaction between introduced rainbow trout and native silverside in a Chilean stream. Environ Biol Fish 86:353-359.

PEARCE J \& S FERRIER. 2000. Evaluating the predictive performance of habitat models developed using logistic regression. Ecological Modelling 133 (3):225-245.

PEREDO-PARADA M, MARTÍNEZ-CAPEL F, GARÓFANO-GÓMEZ V, ATENAS M \& F RIESTRA. 2009. Base de datos Eco-Hidrológica de los ríos de Chile: Una Herramienta de gestión para los ecosistemas acuáticos. Gayana 73 (1): 219-229.

PEREDO-PARADA $\mathrm{M}, \mathrm{F}$ MARTÍNEZ-CAPEL, D QUEVEDO \& A HERNÁNDEZ-MASCARELL. Implementation of an Eco-Hydrological Classification of Chilean Rivers. 2010. Gayana (In press).

PETER L, M GOETHALS, A DEDECKER, W GABRIELS, S LEK \& N DE PAUW. 2007. Applications of artificial neural networks predicting macroinvertebrates in freshwaters. Aquatic Ecology 41:491-508.

PETERS J, B DE BAETS, N VERHOEST, R SAMSON, S DEGROEVE, P DE BECKER \& W HUYBRECHTS. 2007. Random forests as a tool for ecohydrological distribution modelling. Ecological Modelling 207: 304-318. 
CAPÍTULO 5 DISTRIBUCIÓN GEOGRÁFICA POTENCIAL.

PRASAD A, L IVERSON \& A LIAW. 2006. Newer Classification and Regression Tree Techniques: Bagging and Random Forests for Ecological Prediction. Ecosystems 9: 181-199.

R DEVELOPMENT CORE TEAM. 2008. R: A language and environment for statistical computing. R Foundation for Statistical Computing, Vienna, Austria. ISBN 3-900051-07-0, URL http://www.R-project.org.

RABENI C \& K DOISY. 2000. Correspondence of stream benthic invertebrate assemblages to regional classification schemes in Missouri. Journal of the North American Benthological Society 19: 419-428.

RECKNAGEL F. 2003. Ecological Informatics: Understanding Ecology by Biologically-Inspired Computation. Springer, New York, USA. 398 pp.

SANDIN L \& R JOHNSON. 2000. Ecoregions and benthic macroinvertebrate assemblages of Swedish streams. Journal of North American Benthological Society 19:462-474.

SNELDER T, F CATTANEO, A SUREN \& B BIGGS. 2004. Is the River Environment Classification an improved landscape-scale classification of rivers?.Journal of the North American Benthological Society. 23(3): 580-589.

SNELDER T, B BIGGS \& R WOODS. 2005. Improved Eco-Hydrological classification of rivers. River Research and Applications 21: 609-628.

SOWA S, G ANNIS, M MOREY \& D DIAMON. 2007. A gap analysis and comprehensive conservation strategy for riverine ecosystems of missouri. Ecological Monographs 77(3): 301-334.

TIRELLI T \& D PESSANI. 2009. Use of decision tree and artificial neuronal network approaches to model presence/absence of TELESTES MUTICELLUS in piedmont (North-Western Italy). River Research and Applications 25: 1001-1012.

VAN SICKLE J. 1997. Using mean similarity dendrograms to evaluate classifications. Journal of agricultural, Biological and Environmental Statistics 2: 370-388.

VAN SICKLE J \& R HUGHES. 2000. Classification strengths of ecorregions, catchments, and geographic cluster for aquatic vertebrates in Oregon. Journal of North American Benthological Society 19(3): 370-384.

VILA I, L FUENTES \& M CONTRERAS. 1999. Peces Límnicos de Chile. Boletín del Museo de Historia Natural, Chile. 48: 61-75. 
CAPITULO 5 DISTRIBUCIÓN GEOGRÁFICA POTENCIAL. WAITE I, A HERLIHY, D LARSEN, \& D KLEMM. 2000. Comparing strengths of geographic and nongeographic classifications of stream benthic macroinvertebrates in the Mid-Atlantic Highlands, USA. Journal of the North American Benthological Society 19:429-441. 

CAPÍTULO 5 DISTRIBUCIÓN GEOGRÁFICA POTENCIAL.

\subsection{ANEXOS}





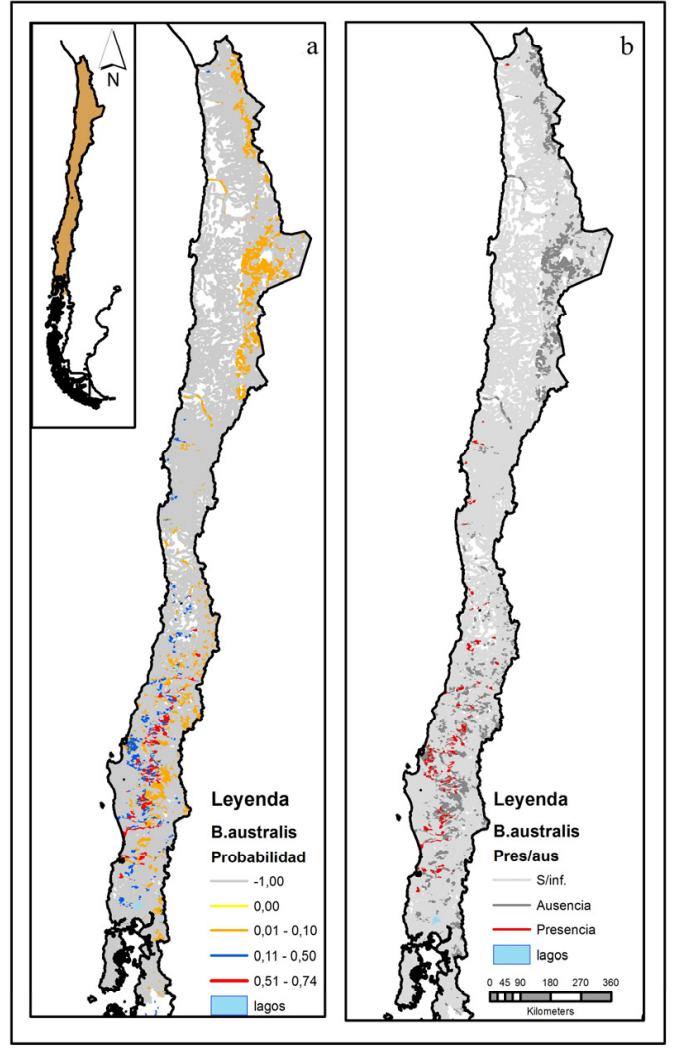

Figura 5.1. Distribución geográfica potencial para la especie B. australis. Probabilidad de ocurrencia (a) y presencia/ausencia (b)

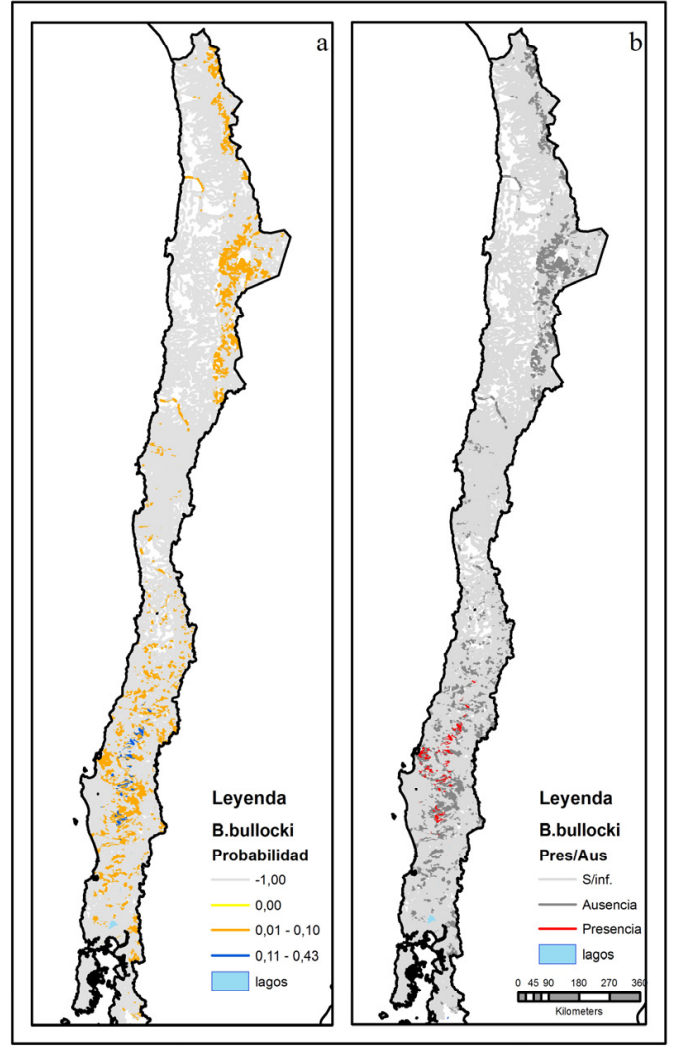

Figura 5.2. Distribución geográfica potencial para la especie B. bullocki. Probabilidad de ocurrencia (a) y presencia/ausencia (b) 


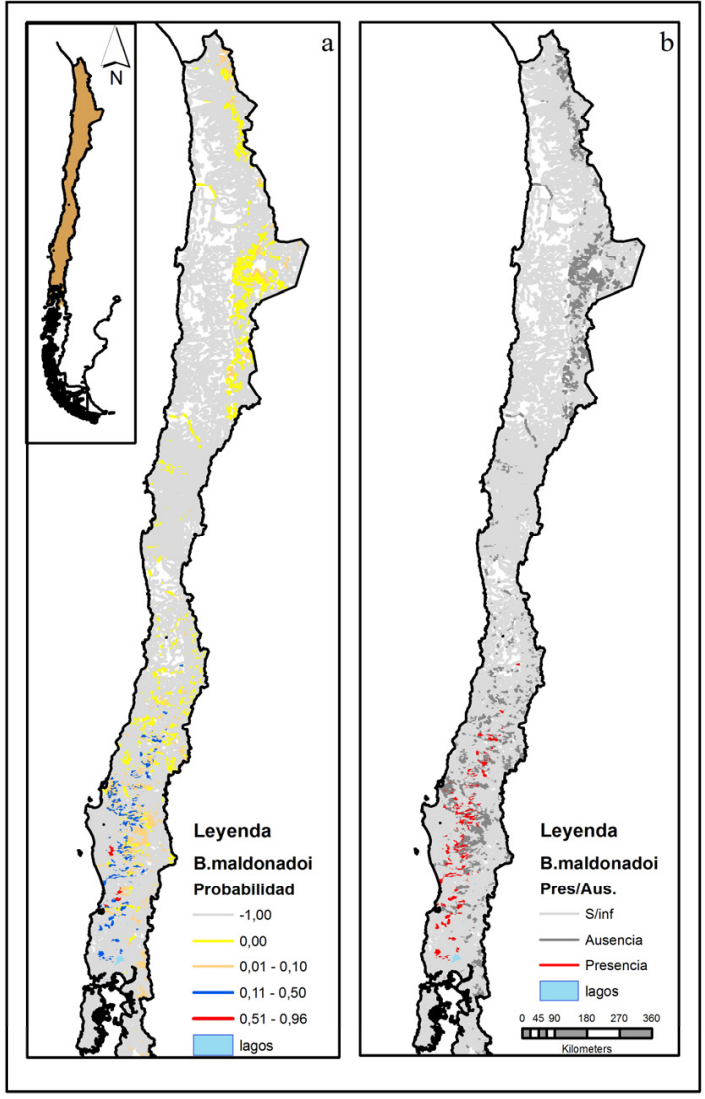

Figura 5.3. Distribución geográfica potencial

$$
\text { para la }
$$
especie

de ocurrencia (a) y presencia/ausencia (b)

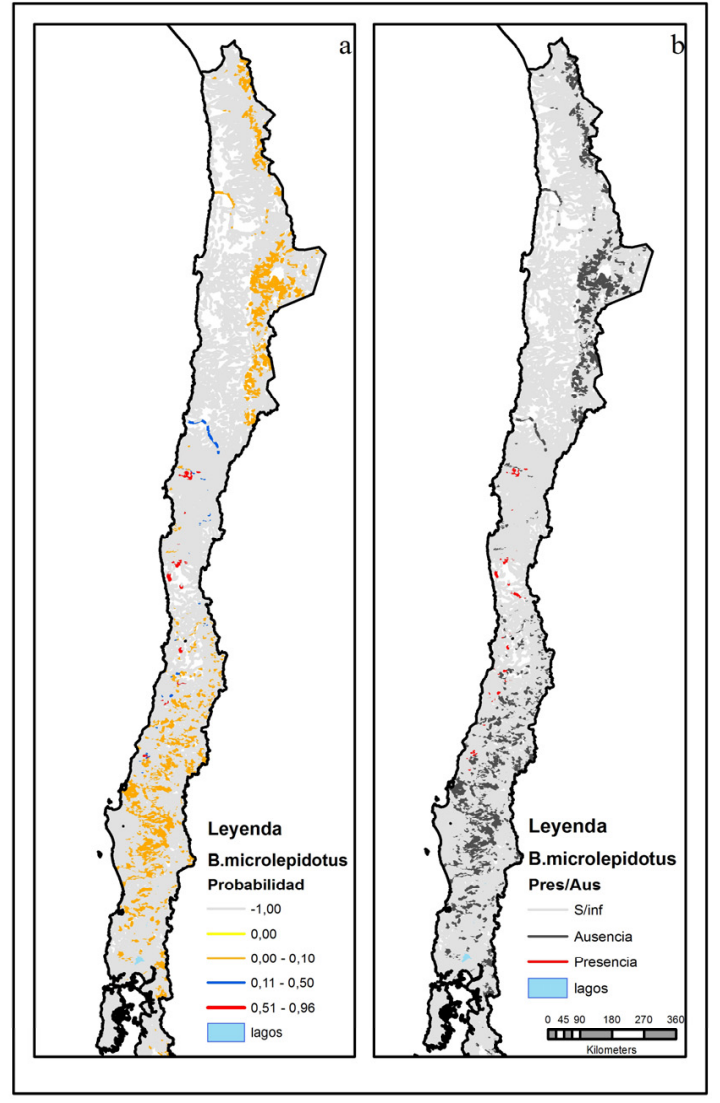

Figura 5.4. Distribución geográfica potencial para la especie B. microlepidotus. Probabilidad de ocurrencia (a) y presencia/ausencia (b) 


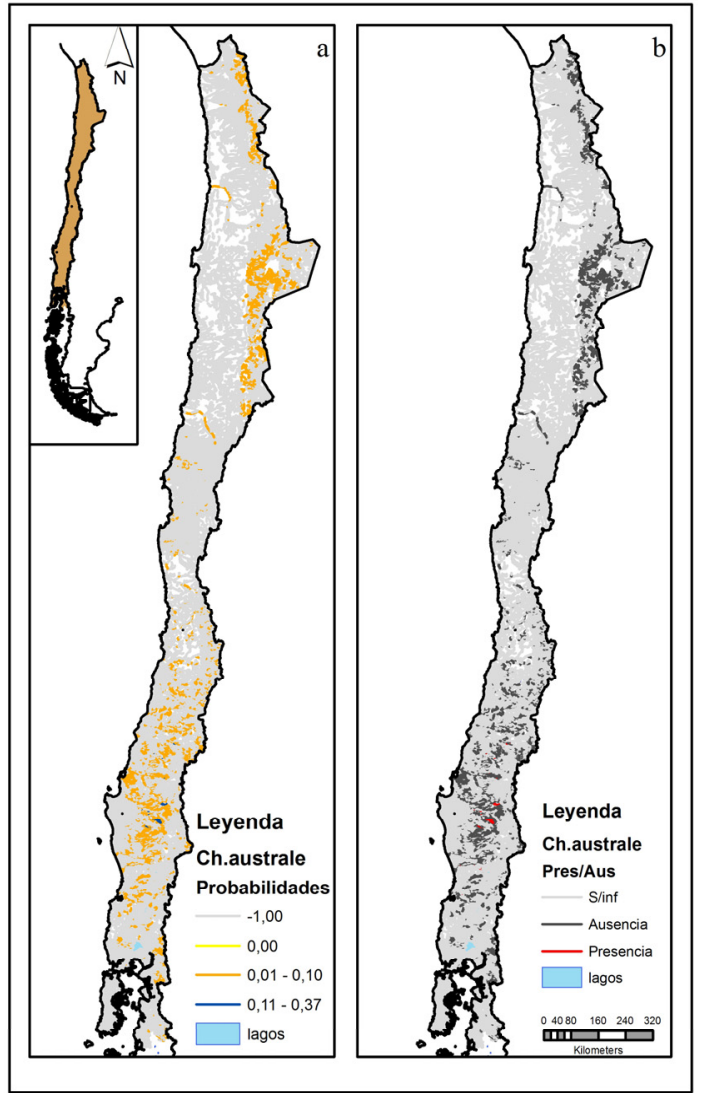

Figura potencial australe. Probabilidad de ocurrencia (a) y presencia/ausencia (b)

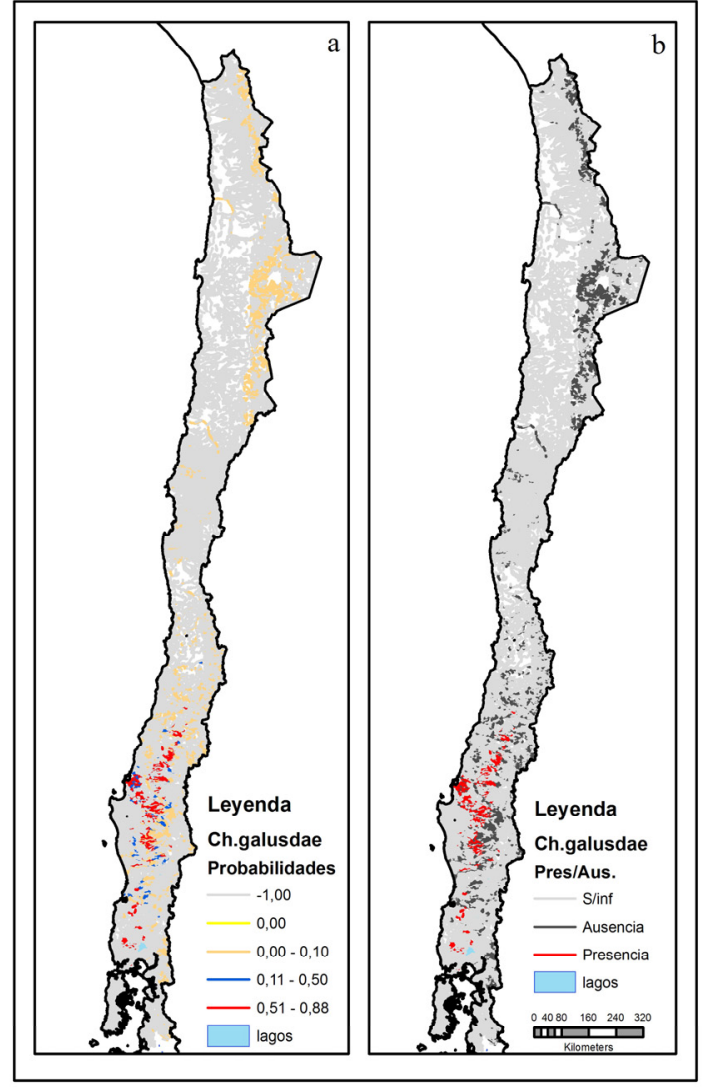

Figura 5.6. Distribución geográfica potencial para la especie Ch. galusdae. Probabilidad de ocurrencia (a) y presencia/ausencia (b) 


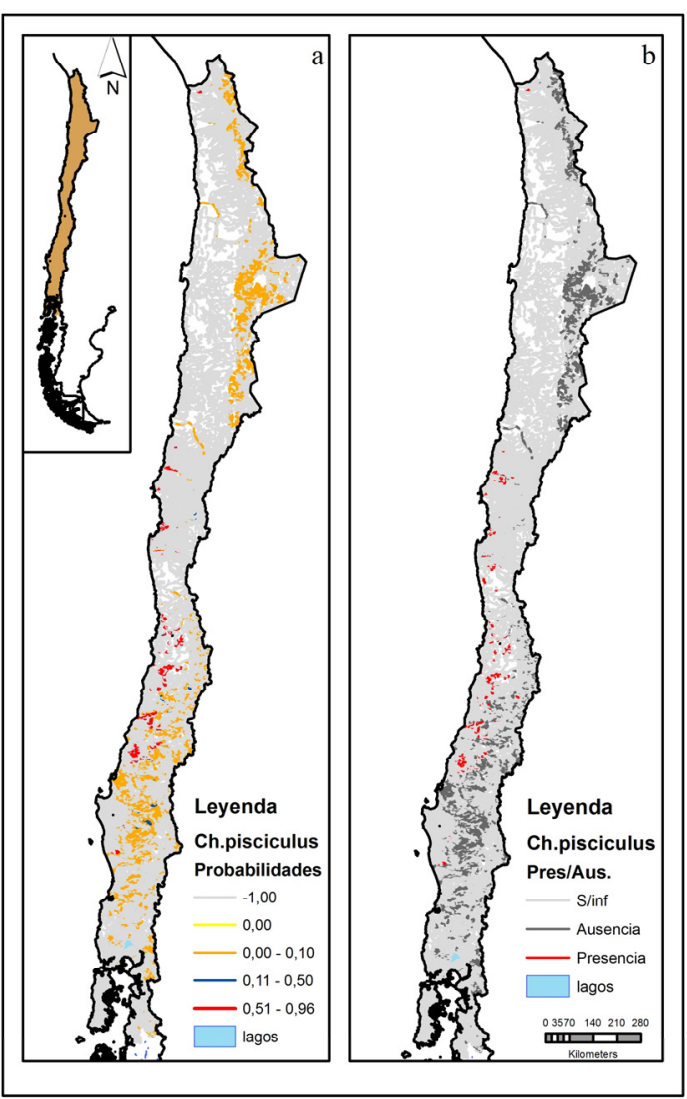

Figura

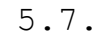

Distribución

geográfica potencial para la especie Ch. (a) y presencia/ausencia (b)

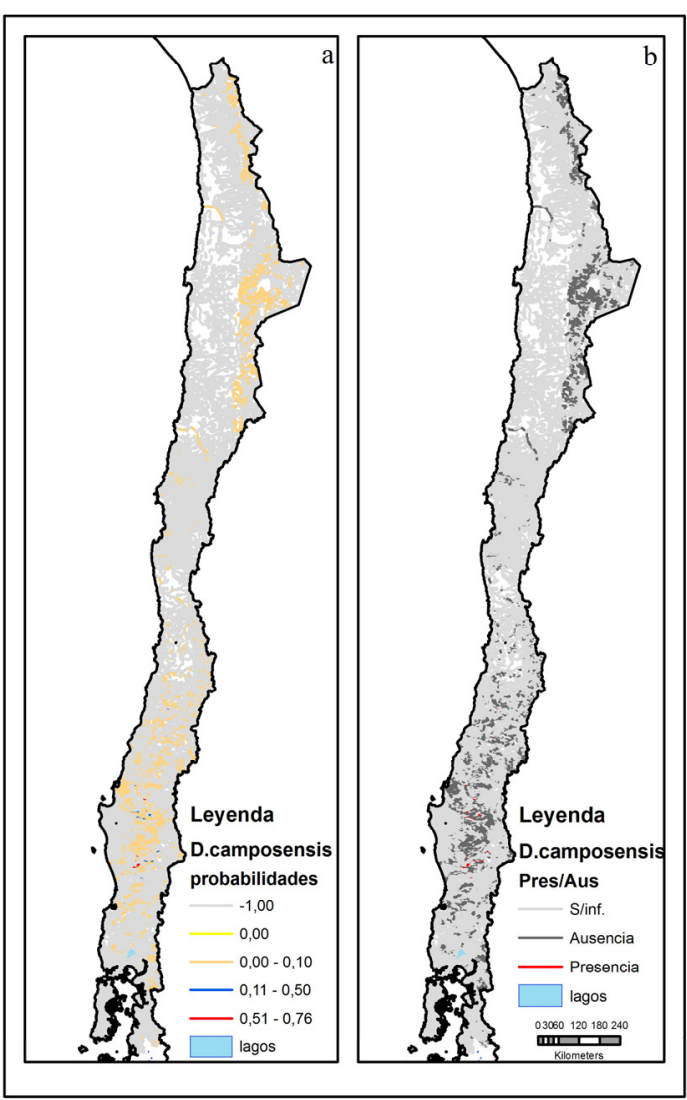

Figura 5.8. Distribución geográfica potencial para la especie D.camposensis. Probabilidad de ocurrencia (a) y presencia/ausencia (b) 


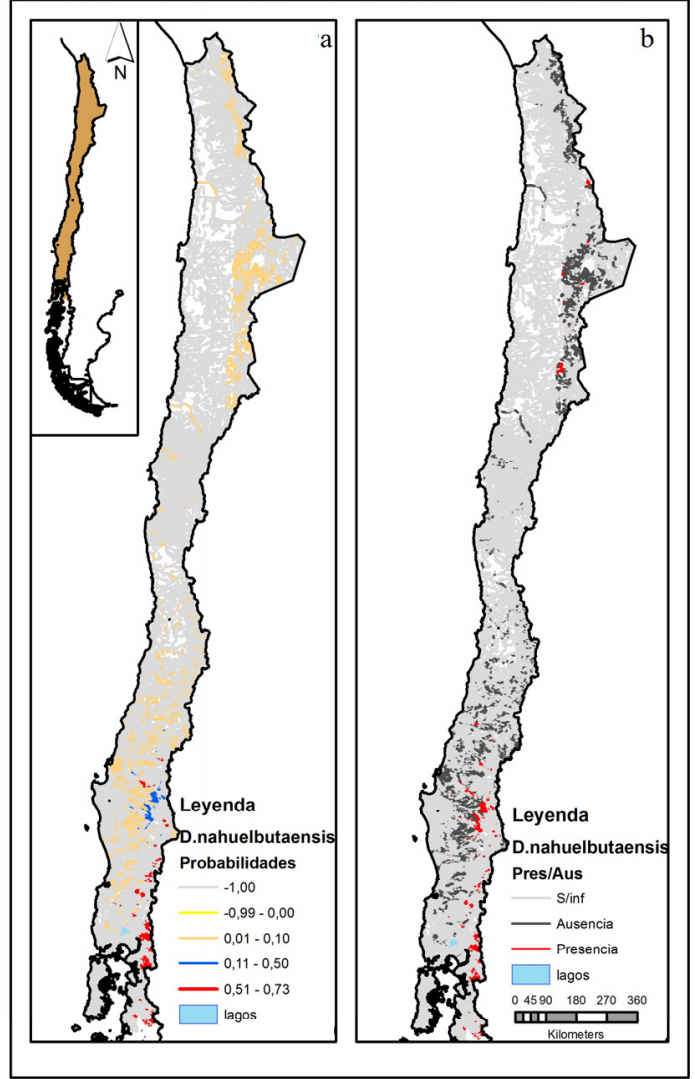

Figura

5.9. Distribución

potencial para nahuelbutaensis. la Probabilidad

ocurrencia (a) y presencia/ausencia
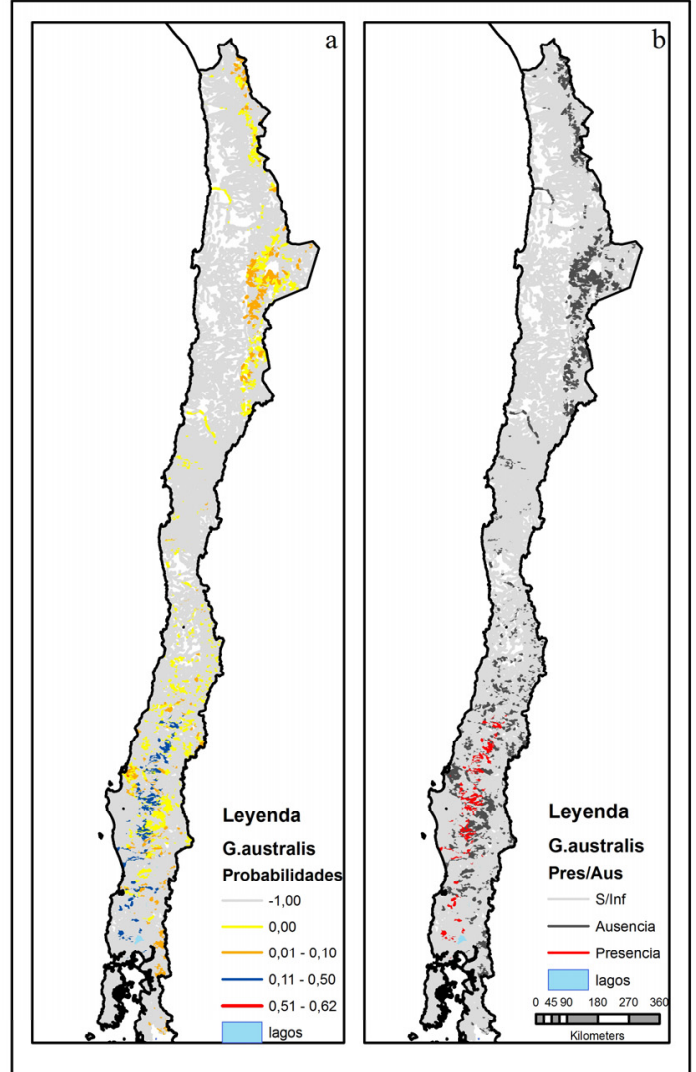

Figura 5.10. Distribución geográfica potencial para la especie G. ausralis. Probabilidad de ocurrencia (a) y presencia/ausencia (b) 


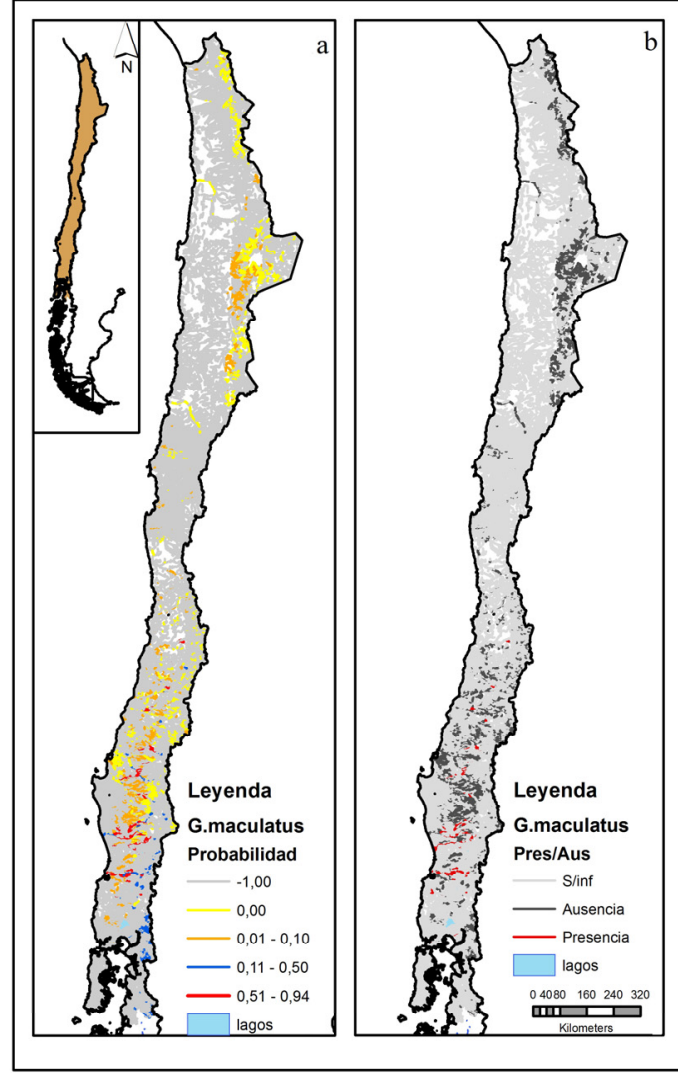

Figura 5.11. Distribución geográfica potencial para la especie G. maculatus. Probabilidad de ocurrencia (a) y presencia/ausencia (b).

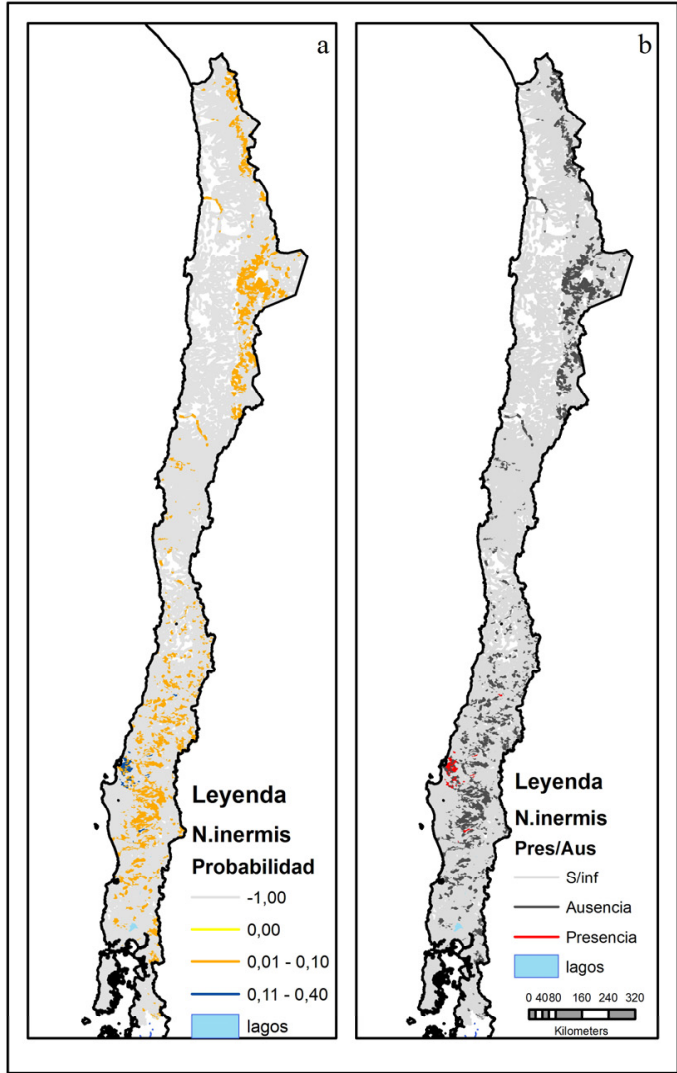

Figura 5.12. Distribución geográfica potencial para la especie N. inermis. Probabilidad de ocurrencia (a) y presencia/ausencia (b) 


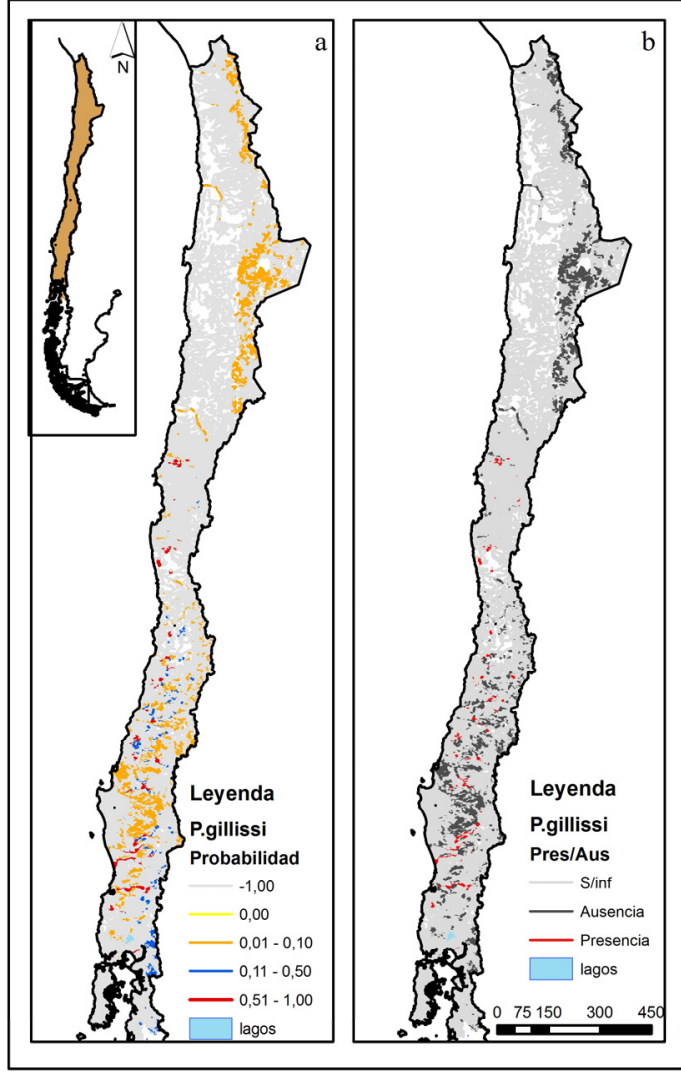

Figura 5.13. Distribución geográfica potencial para la especie P. gillissi. Probabilidad de ocurrencia (a) y presencia/ausencia (b) .

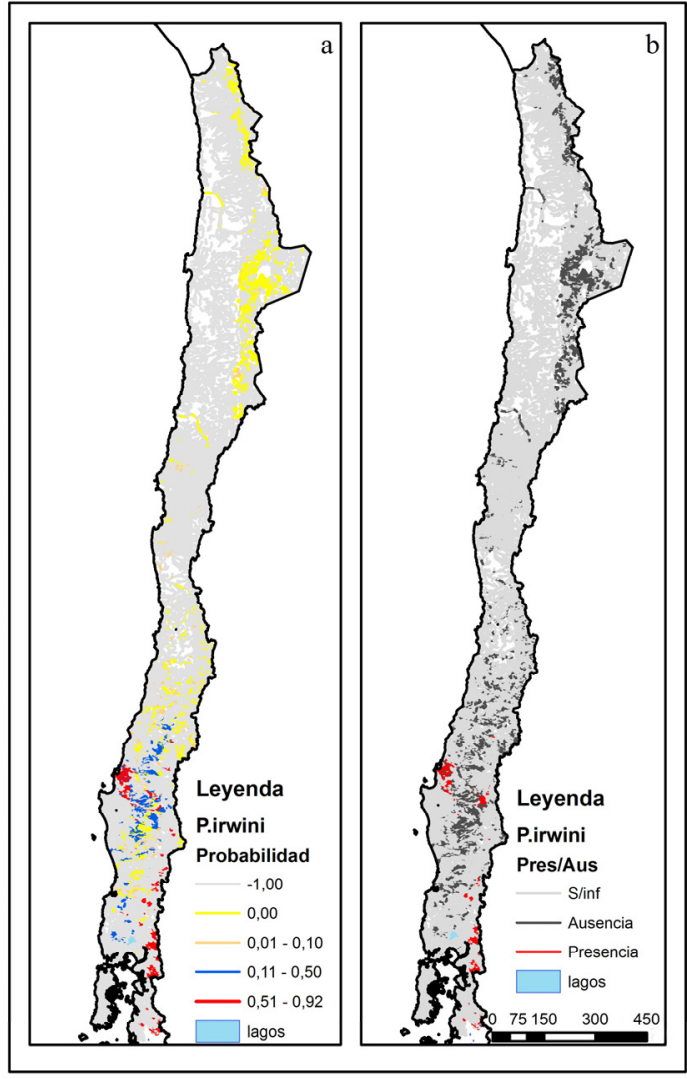

Figura 5.14. Distribución geográfica potencial para la especie P. irwini. Probabilidad de ocurrencia (a) y presencia/ausencia (b) . 


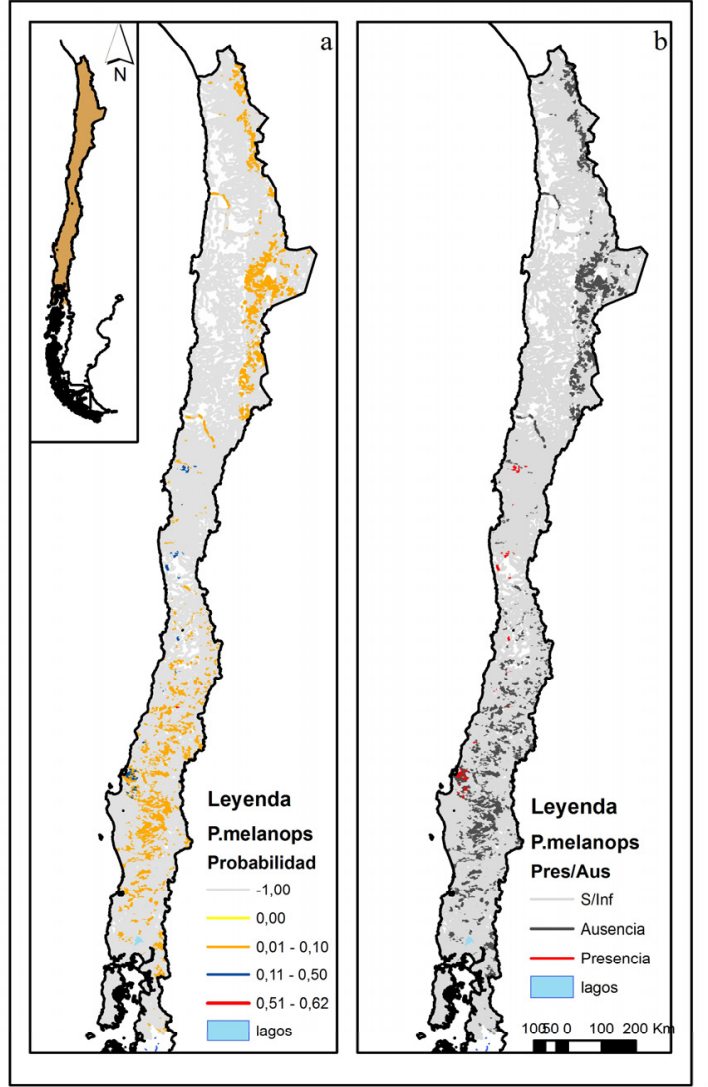

Figura 5.15. Distribución geográfica potencial para la especie P. melanops. Probabilidad de ocurrencia (a) y presencia/ausencia (b) .

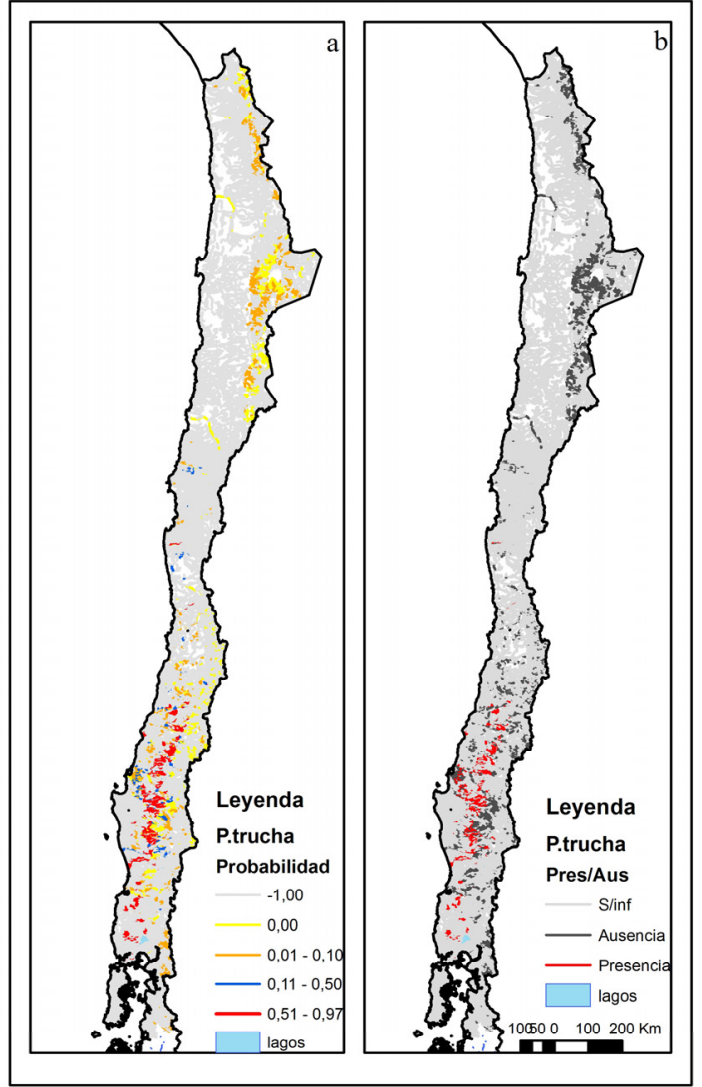

Figura 5.16. Distribución geográfica potencial para la especie P. trucha. Probabilidad de ocurrencia (a) y presencia/ausencia (b) . 


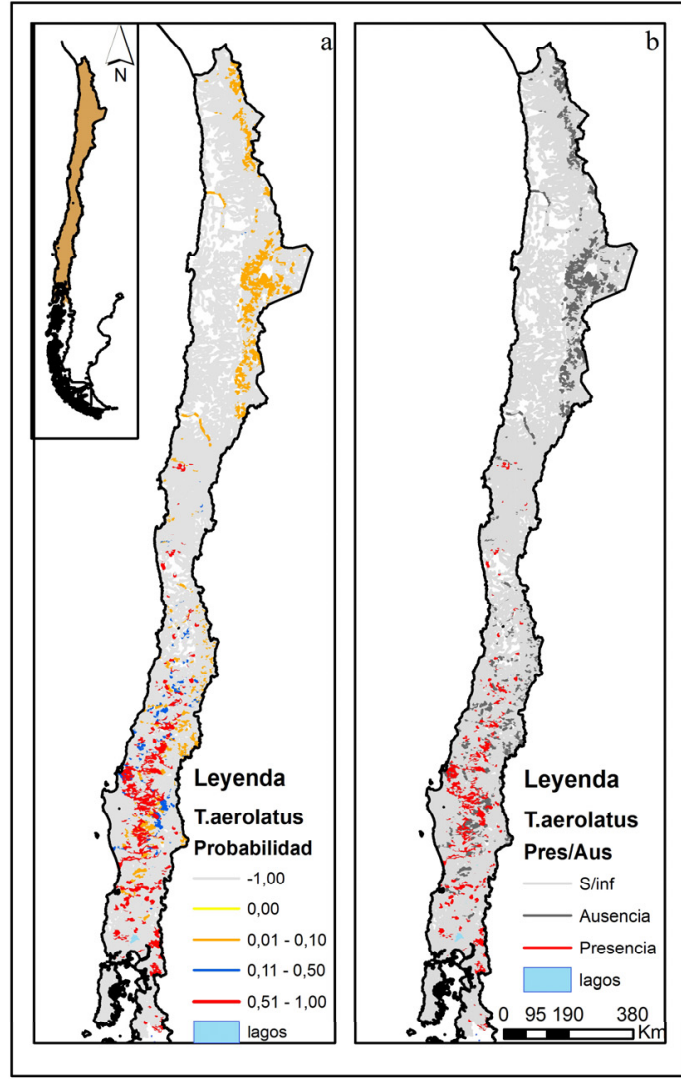

Figura 5.17. Distribución geográfica potencial para la especie $T$. aerolatus. Probabilidad de ocurrencia (a) y presencia/ausencia (b) .

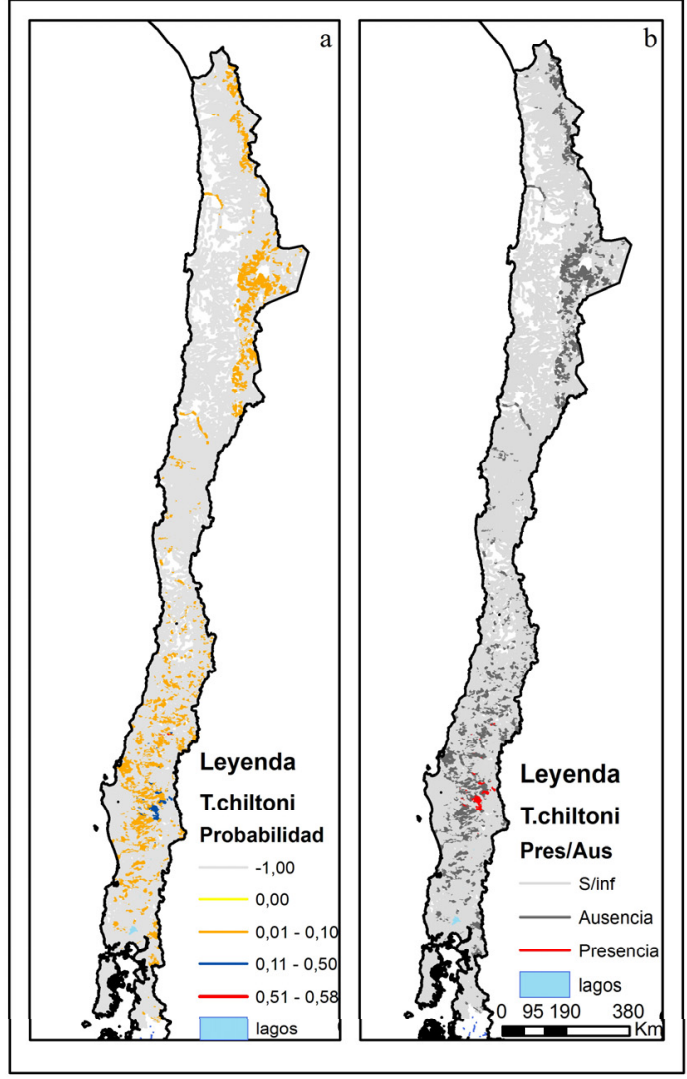

Figura 5.18. Distribución geográfica potencial para la especie T.chiltoni. Probabilidad de ocurrencia (a) y presencia/ausencia (b) . 

Capitula 6

Conclusiones generales 

Con el objetivo de proveer un marco espacial para la gestión ambiental de los ríos en Chile, se implementó una clasificación Eco-Hidrológica (RECChile) basada en la superposición jerárquica de 6 factores controladores (Clima, Origen de Flujo, Geología, Posición Relativa de Subsubcuenca, Uso del Suelo y Pendiente del tramo). La elección de estos factores se basó en la hipótesis de que el clima, la topografía y la geología suelo son las causantes principales de la variación espacial del patrón hidrológico de un río a una escala determinada. Como resultado de la clasificación se obtuvo una codificación de seis dígitos para cada tramo de río, en donde cada dígito corresponde a un factor controlador y el valor del dígito indica la categoría a la que pertenece dentro de cada factor.

La REC-Chile es una clasificación que permite describir diversos patrones ecohidrológicos de los ríos de Chile mediante la superposición de factores controladores adecuados. Entre sus características más importantes, destacan la propiedad de poder clasificar los ríos a distintas escalas espaciales, la posibilidad de extrapolar información a cuencas sin información y la capacidad de manejar a los ríos como una red hídrica considerando la continuidad longitudinal, y al mismo tiempo agregando las propiedades de la cuenca al ir aumentando el área vertiente permitiendo detectar cambios de una subcuenca a otra. La REC-Chile está implementada en un ambiente SIG, lo que facilita su interpretación geográfica y el cambio a distintas escalas de trabajo.

Sin lugar a dudas, una de las mayores ventajas de la REC-Chile es que permite definir niveles de clasificación según los factores que se han 
REF_REF256155293 IR IH |CAPÍtULO 6\} CONCLUSIONES GENERALES superpuesto. Estos niveles tienen la particularidad de dotar a la REC-Chile de una flexibilidad para clasificar ya que, por una parte, permiten describir diversos patrones ecohidrológicos y por otra definen diversas escalas espaciales de clasificación, según el nivel de clasificación deseado.

En esta tesis se mostró que el primer nivel de clasificación, compuesto únicamente por el factor Clima, tiene la capacidad de discriminar órdenes de magnitud de los caudales medios a una macroescala. El segundo factor, compuesto por la superposición jerárquica de los factores Clima y Origen de Flujo, discrimina la estacionalidad del flujo a mesoescala. El tercer nivel de clasificación, definido como la superposición de los factores Clima, Origen de Flujo y Geología discrimina aspectos de la calidad geoquímica de las aguas. (Capítulo 3). Asimismo, el sexto nivel de clasificación discrimina en forma satisfactoria aspectos ecológicos como la distribución espacial de las especies ícticas nativas de Chile (Capítulo 5).

La REC-Chile no solo permite trabajar con niveles de clasificación, sino que la combinación jerárquica de factores controladores también es capaz de discriminar patrones hidrológicos. Esto se mostró al combinar los factores Clima y Posición Relativa de Subsubcuenca para discriminar patrones de magnitud del flujo medio mensual y sus variaciones extremas (Capítulo 3).

La interpretación de las clases REC-Chile es sencilla, lo que permite inferir información ecohidrológica en los tramos de ríos a partir de su codificación. Por ejemplo, es posible realizar una descripción sencilla del hidrograma medio anual en régimen natural, por tramos de río, mediante la estimación 
de estos parámetros: magnitud del caudal medio anual, magnitud de los caudales mensuales máximos y mínimos, y por último la estacionalidad a través de los meses de ocurrencia de estos caudales. También se realizó una interpretación de la calidad geoquímica del agua determinando los valores medios y extremos de la conductividad eléctrica. Esta interpretación puede realizarse en forma conjunta para los niveles de clasificación en análisis. Así, para un tramo de río, la clasificación a un tercer nivel permite interpretar la magnitud del caudal anual medio, su estacionalidad y valores medios de conductividad eléctrica.

Las características de la REC-Chile, mencionadas anteriormente, permiten utilizarla como plataforma en la gestión de los ecosistemas fluviales. Entre ellas, la posibilidad de trabajo a distintas escalas espaciales permitió recopilar en una única base de datos Eco-Hidrológica información de diferentes escalas espaciales, como información fluviométrica, fisicoquímica, sedimentológica y la escasa información de las especies ícticas nativas de Chile como se mostró en el Capítulo 4.

La aplicación de la REC-Chile para modelos de distribución de especies dulceacuícolas nativas permitió estimar la distribución geográfica potencial de 32 especies nativas utilizando las clases REC-Chile como las variables de entrada en modelos de predicción presencia/ausencia.

Los modelos resultantes, mediante Random Forests, tuvieron un comportamiento general considerado como bueno según los distintos indicadores de evaluación utilizados, sin embargo 14 de ellos presentaron 
REF_REF256155293 IR IH |CAPÍtUlo 6\} CONCLUSIONES GENERALES comportamientos erráticos debido a la baja prevalencia inherente a las especies nativas, cuyos datos son muy escasos y se conoce poco sobre su distribución geográfica. Los 18 modelos restantes, presentaron resultados más estables, por lo tanto, solo para estas especies se desarrollaron los mapas de distribución geográfica potencial (en anexos del Capítulo 5).

A partir de estos modelos de distribución espacial, y considerando la interpretación hidrológica y fisicoquímica de las aguas realizada para las clases REC-Chile, sería posible determinar las zonas de conservación y relocalización para cada una de estas especies, así como sus preferencias de hábitat a nivel de mesoescala. Esta es una de las líneas abiertas que se desea desarrollar a partir de esta tesis doctoral.

Los potenciales usos de la REC-Chile en la gestión de los ecosistemas fluviales son extensos. A los ya mencionados anteriormente y su directa aplicación, se suman aquellos orientados a determinar el estado ecológico actual de cada tramo de río, y definir sus tramos de referencia para desarrollar planes de restauración fluvial. Así también, la REC-Chile puede ser utilizada como marco para definir las metodologías idóneas para la determinación del régimen de caudales ecológicos considerando las características hidrológicas, geomorfológicas y bióticas de cada tramo de río. Esta constituye la segunda línea de investigación que desea desarrollarse con gran intensidad a partir de esta tesis

A modo general, la REC-Chile es una clasificación de los ríos de Chile que permite clasificarlos bajo distintos objetivos y escalas espaciales, lo que le 
da un carácter multifuncional imprescindible para una gestión ambiental. Finalmente esperamos que la clasificación REC-Chile se constituya en una herramienta para la conservación y gestión de los ecosistemas fluviales en Chile. 